\title{
Uncovering the intrinsic size dependence of hydriding phase transformations in nanocrystals
}

\author{
Rizia Bardhan ${ }^{1 \dagger}$, Lester O. Hedges ${ }^{1 \dagger}$, Cary L. Pint ${ }^{2,3}$, Ali Javey ${ }^{2,3}$, Stephen Whitelam ${ }^{1 \star}$ \\ and Jeffrey J. Urban ${ }^{1 \star}$
}

\begin{abstract}
A quantitative understanding of nanocrystal phase transformations would enable more efficient energy conversion and catalysis, but has been hindered by difficulties in directly monitoring well-characterized nanoscale systems in reactive environments. We present a new in situ luminescence-based probe enabling direct quantification of nanocrystal phase transformations, applied here to the hydriding transformation of palladium nanocrystals. Our approach reveals the intrinsic kinetics and thermodynamics of nanocrystal phase transformations, eliminating complications of substrate strain, ligand effects and external signal transducers. Clear size-dependent trends emerge in nanocrystals long accepted to be bulk-like in behaviour. Statistical mechanical simulations show these trends to be a consequence of nanoconfinement of a thermally driven, first-order phase transition: near the phase boundary, critical nuclei of the new phase are comparable in size to the nanocrystal itself. Transformation rates are then unavoidably governed by nanocrystal dimensions. Our results provide a general framework for understanding how nanoconfinement fundamentally impacts broad classes of thermally driven solid-state phase transformations relevant to hydrogen storage, catalysis, batteries and fuel cells.
\end{abstract}

A first-order phase transformation begins with nucleation of a new, stable phase in a background of an old, metastable one. Nucleation is an activated process: thermal fluctuations are required to overcome a free energy barrier and generate a critical nucleus of the new phase. In defect-free bulk solids, this barrier is determined solely by the free energy difference $(\Delta G)$ and surface tension $(\sigma)$ between phases ${ }^{1}$, and scales as $\sigma^{3} /(\Delta G)^{2}$. However, in nanocrystals, interactions between the new phase and nanocrystal surfaces can profoundly influence the thermodynamics and kinetics of nucleation. Thus, understanding phase changes in nanocrystals requires precise control of surface-to-volume ratio, and a direct readout of the progress of the phase transformation ${ }^{2-5}$. Although the experimental understanding of how nanocrystal size controls the rates and energies of metal hydride formation and decomposition has advanced rapidly in recent years ${ }^{6}$, it remains limited to engineered samples and specific geometries, owing to challenges both in directly probing phase change at the nanoscale and in preparing uniform samples over a series of sizes. Recent advances have demonstrated the promise of nanoscale metal hydrides as sensors ${ }^{7}$ and $\mathrm{H}_{2}$ storage media ${ }^{8,9}$, but quantification of the size dependence of these processes has remained largely inaccessible, hindering rational optimization of these properties. However, combining new optical methodology with monodisperse nanocrystals synthesized by bottom-up approaches provides a means of accurately monitoring $\mathrm{H}_{2}$-induced phase transitions as a function of crystal size. Existing techniques for examining metal to metal hydride transformations (gravimetric, volumetric, X-ray diffraction (XRD)) possess several key drawbacks-slow kinetics, requirement of large sample volumes, limited sensitivity, low temporal and spatial resolution, and deviations from isothermal conditions due to evolved reaction heat. Optical methodologies, based on modifications in transmission response $\mathrm{e}^{10,11}$ and localized surfaceplasmon resonance ${ }^{6,12-14}$ (LSPR), have recently yielded important insights into hydrogen uptake and release in nanoscale systems by providing enhanced sensitivity and temporal resolution. However, they are often suitable only for thin-film geometries ${ }^{10,11}$, or require coupling to external gold nanoantennas for signal readout $t^{6,12}$.

Here we demonstrate a new optical approach for rapid, direct monitoring of minuscule alterations in metal nanocrystal luminescence during $\mathrm{H}_{2}$ sorption to uncover the size dependence of the intrinsic thermodynamics and kinetics of hydriding/dehydriding phase transformations. Systematic evaluation of how nanocrystal size impacts kinetics and thermodynamics of conversion (Figs 1-5), and the enthalpic and entropic driving forces for $\mathrm{H}_{2}$ storage (Supplementary Table $\mathrm{S} 1$ ), reveal clear trends that are understood by interpreting hydriding as a nanoconfined, thermally driven phase transformation. Moreover, whereas top-down fabrication approaches commonly used are constrained to production of micrometre-sized crystals ${ }^{13}$, the $\mathrm{Pd}$ nanocubes studied here are synthesized by wet chemistry (Fig. 1a-d) and are of narrow size distribution (Supplementary Fig. S17) with controllable size and surface termination (Supplementary Fig. S2); they are all single-crystalline objects possessing clear $\{100\}$ facets. We observe a pronounced decrease in Pd luminescence as nanocubes hydride to form $\mathrm{PdH}_{0.6}$; during dehydriding, nanocubes regain their luminescence (Fig. 1e,f and Supplementary Fig. S5). We use these optical signatures of phase transformations, in conjunction with simulation, to understand how nanocrystal size impacts the energetics of metal hydride formation and decomposition. Our in situ luminescence-based technique eliminates the need for coupled

\footnotetext{
${ }^{1}$ Molecular Foundry, Materials Sciences Division, Lawrence Berkeley National Laboratory, Berkeley, California 94720, USA, ${ }^{2}$ Department of Electrical Engineering and Computer Sciences, University of California, Berkeley, California 94720, USA, ${ }^{3}$ Berkeley Sensor and Actuator Center, University of California, Berkeley, California 94720, USA. †These authors contributed equally to this work. ${ }^{\dagger}$ Present addresses: Department of Chemical and Biomolecular Engineering, Vanderbilt University, Nashville, Tennessee 37235, USA (R.B.); Department of Mechanical Engineering, Vanderbilt University, Nashville, Tennessee 37235, USA (C.L.P.). *e-mail: swhitelam@lbl.gov; jjurban@lbl.gov
} 


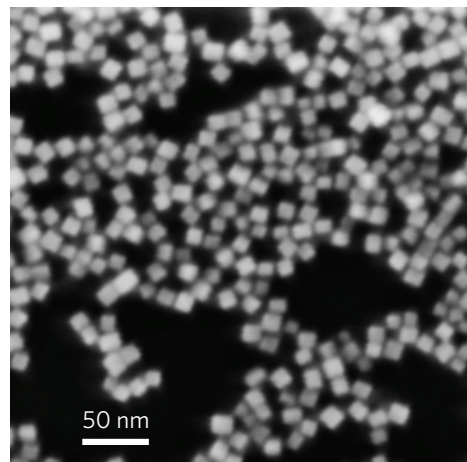

d

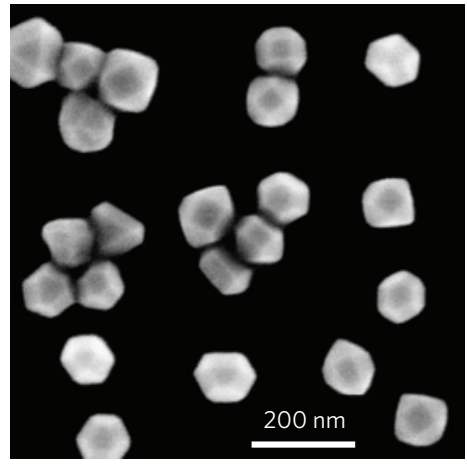

b

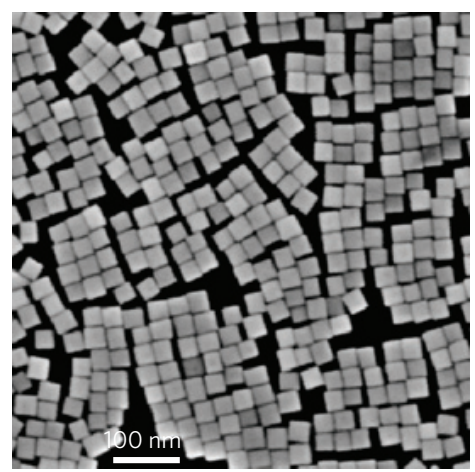

e

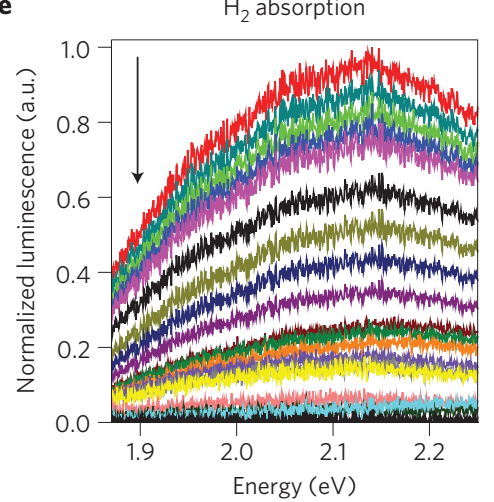

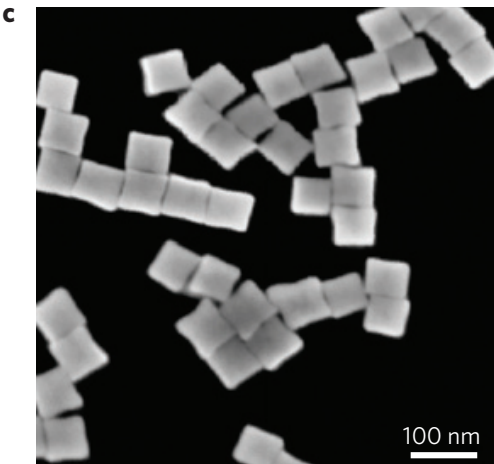

f

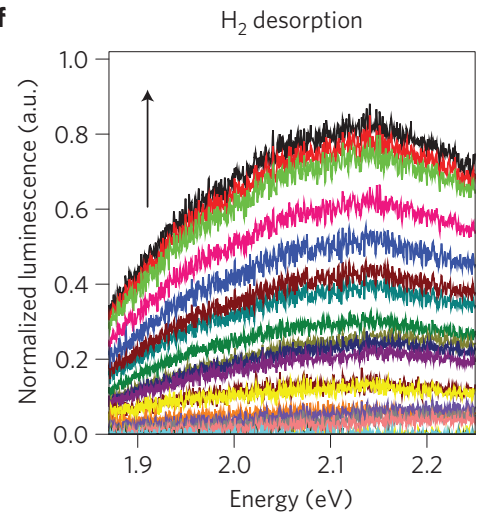

Figure 1 | Pd nanocube characterization and luminescence. a-d, Scanning electron micrographs of Pd nanocubes of side length $14 \pm 1 \mathrm{~nm}$ (a), $32 \pm 2 \mathrm{~nm}$ (b), $65 \pm 4 \mathrm{~nm}$ (c) and $110 \pm 12 \mathrm{~nm}$ (d). e,f, Luminescence spectra of $14 \mathrm{~nm}$ Pd nanocubes at $22^{\circ} \mathrm{C}$ decreasing during hydriding (e) and increasing during dehydriding (f). Data, for each set of conditions, are normalized by dividing luminescence values by the luminescence of the metal phase.

optical transducers to provide Pd signal enhancement. Moreover, in contrast to LSPR-based sensing, which requires exquisite fabrication of plasmonic antennas ${ }^{14}$ or $\mathrm{SiO}_{2}$ dielectric spacers ${ }^{6}$, which screen the changes in electronic structure under reactive transformation, luminescence provides a direct readout of $\mathrm{H}_{2}$ uptake and transformation in $\mathrm{Pd}$ nanocrystals. Luminescence from $\mathrm{Pd}$ and other noble metals arises from a combination of radiative decay of surface plasmons, and inter- and intraband excitations of plasmons ${ }^{15,16}$. We also quantified the luminescence quantum yield of all sizes of nanocube (Supplementary Section SII-A and Fig. S1). Thus, this luminescence approach can be generalized to other nanocrystal systems ${ }^{17}$ for various technologies by adjusting the wavelengths used.

Hydriding/dehydriding experiments were performed using a custom-built gas-flow optical system (Supplementary Fig. S4) with nanocrystal films deposited on quartz substrates (see Methods and Supplementary Section SI). Pd nanocrystal size was tuned from 14 to $110 \mathrm{~nm}$, while maintaining simple geometric scaling of surface-area-to-volume ratios within $\sim 5 \%$ (Supplementary Section SII-E). This luminescence-based approach, unlike LSPR-based techniques, can be employed to understand hydriding/dehydriding behaviour in nanocrystals $<10 \mathrm{~nm}$ (Supplementary Fig. S3) without use of plasmonic antennas for signal enhancement. However, at sizes $<10 \mathrm{~nm}, \mathrm{Pd}$ typically attains a spherical shape terminated by [111] planes, which have very different energetics of $\mathrm{H}_{2}$ dissociation and diffusion, compared with cubic nanocrystals that are bound by [100] planes (Supplementary Fig. S2 and Supplementary Section SII-C). Owing to these differences, spherical Pd nanocrystals $(<10 \mathrm{~nm})$ are not compared in the size range discussed here. Pd nanocrystal crystallinity and size-dependent changes in electronic structure were verified for all sizes (Supplementary Fig. S2). Nanocrystal luminescence decreased during hydriding, and recovers during dehydriding (Fig. 1 and
Supplementary Fig. S5). As Pd metal transforms to $\mathrm{PdH}_{x}$, the density of states near the Fermi energy decreases, resulting in diminished $s p$-electron and $d$-hole recombination and a decrease in luminescence. This phenomenon is reversed during dehydriding ${ }^{15,18}$ (Supplementary Section SII-B).

By measuring luminescence as $\mathrm{H}_{2}$ pressure was gradually increased and then decreased, we obtained pressure-luminescence hysteresis loops for a series of nanocrystal sizes and temperatures (Fig. 2a and Supplementary Figs S7 and S19). A luminescence of 1 corresponds to the metal, and 0 to the metal hydride phase, respectively. Curves are normalized by mass of nanocrystals deposited, and resemble established pressure-composition $(P-C)$ isotherms for $\mathrm{PdH}$ system ${ }^{19}$. At low pressure, in the metal rich phase, small changes in observed luminescence indicate fluctuations of the hydride phase. At larger pressures a sharp decrease in luminescence signals transformation to the metal hydride phase. A plateau at constant pressure is observed where the two phases coexist. These transformations were reversed following a decrease in pressure, with accompanying hysteresis. Size- and temperature-dependent studies reveal that hysteresis diminishes and phase transformations become faster with decreasing nanocrystal size and increasing temperature. As we shall discuss, such behaviours provide critical information about the mechanism underlying nanocube phase transformation.

To interpret our experimental data we simulated the threedimensional Ising model on a cubic lattice ${ }^{20}$. We consider the up- and down- spin states to represent the metal and metal hydride phases, respectively (see Methods and Supplementary Section SI). The Ising model is a prototypical model of phase change, and does not resolve details of the system in question. For instance, we do not explicitly resolve hydrogen molecules or their interactions, or account for lattice elasticity ${ }^{21-25}$. However, we shall show that several important experimental trends can 
a

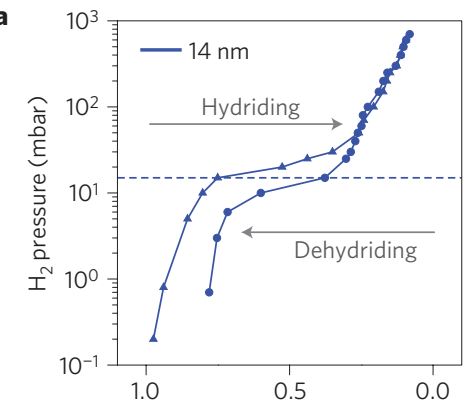

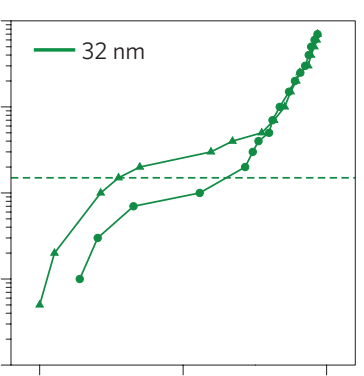

0.5
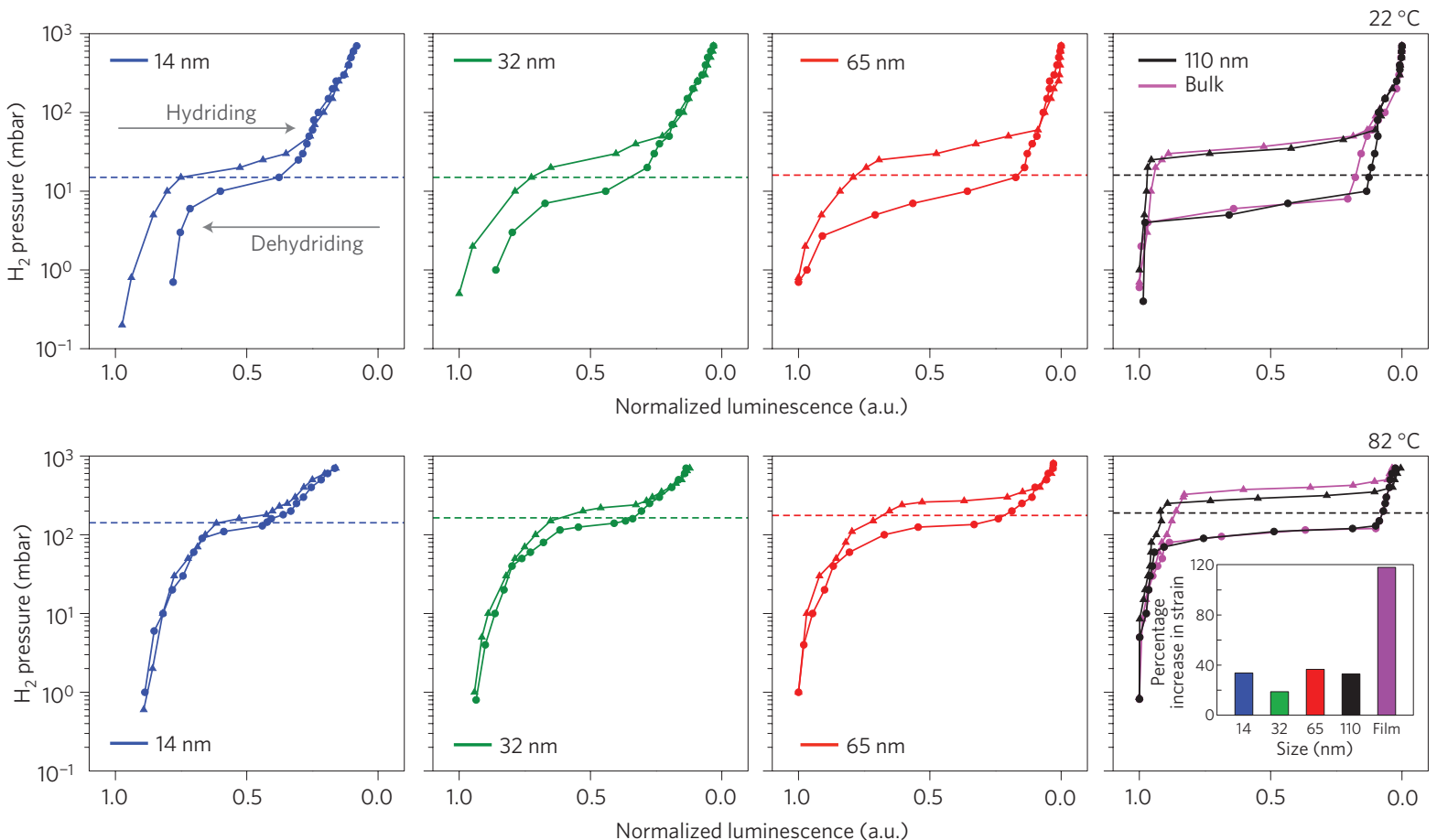

b
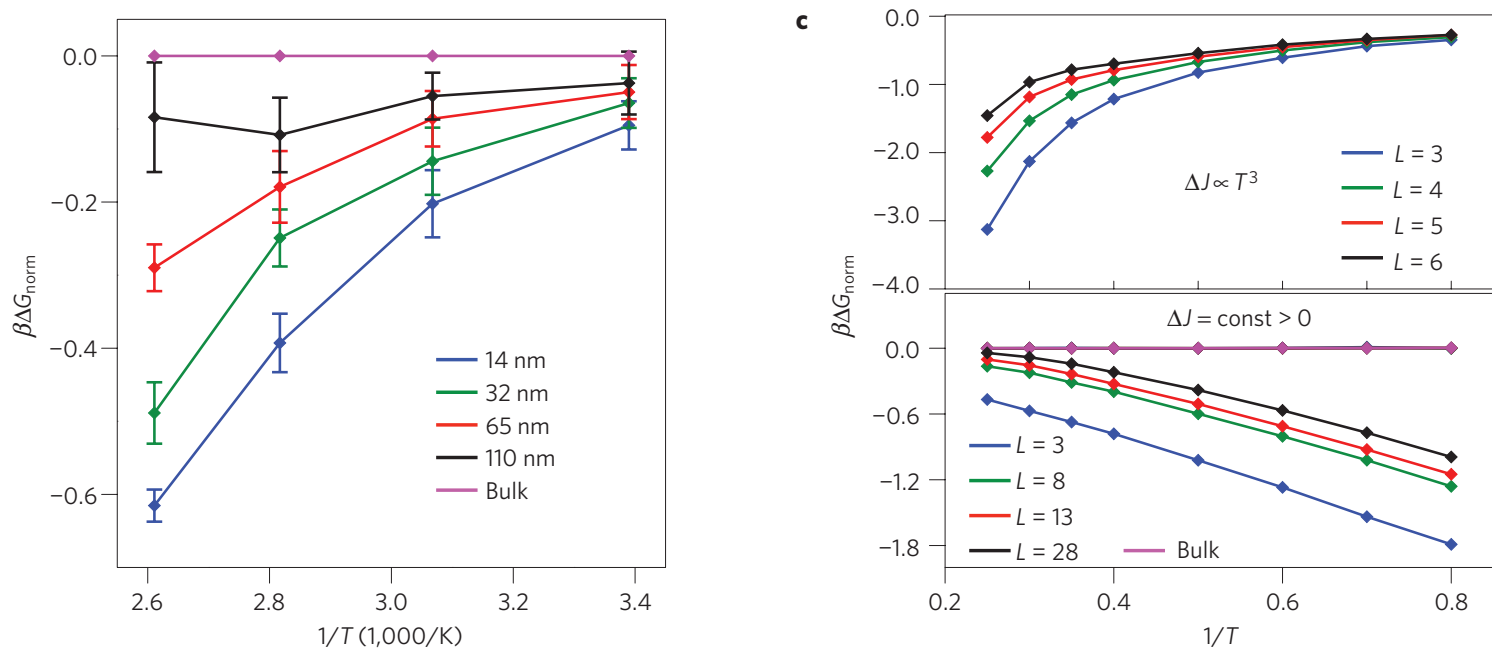

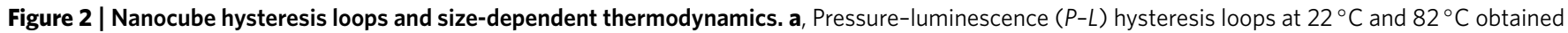
by hydriding and dehydriding Pd nanocubes of four sizes (bulk Pd curves shown as reference). Horizontal dotted lines show calculated coexistence pressures $P_{\text {mid }}$. Inset: percentage increase in strain calculated from full-width at half-maximum of XRD peaks before and after hydriding of nanocubes and $65 \mathrm{~nm}$ Pd film. Detailed calculations are shown in Supplementary Table S3, and Figs S22 and S23. b, Bulk-normalized Van't Hoff plots for nanocubes (vertical axis quantifies the difference in $P_{\text {mid }}$ between nanocubes and bulk samples, $\beta \Delta G_{\text {norm }}=\ln \left(P_{\text {mid }} / P_{\text {mid }}\right)$ ). Here $\beta$ indicates the inverse temperature, $1 / T$. Error bars are calculated from 5 independent measurements. c, Analogous data from Ising model magnetic field-magnetization hysteresis loops:

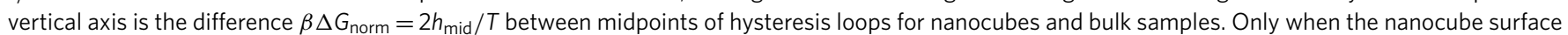
preference $\Delta J$ for one phase increases sufficiently strongly with temperature (upper panel) are experimental trends recovered. The choice $\Delta J \propto T^{3}$ is indicative only. A constant bias $\Delta J$ (lower panel) gives a different trend, and when nanocube surfaces prefer neither phase $(\Delta J=0$, not shown) the midpoint magnetic field remains pinned to the bulk value. Comparison of $\mathbf{b}$ and $\mathbf{c}$ suggests that, experimentally, nanocube surfaces favour the hydride phase, and do so more strongly as temperature increases.

be recovered by representing the observed transformations as conventional, first-order phase transitions enclosed in a finite box. The scaled magnetic field $2 h / T$ is equivalent to the logarithm of experimental pressure, and can be varied to drive the system from one phase to another. We considered both bulk systems and finite nanocubes of side length $L$; in the latter, $\Delta J$ indicates the energetic preference of cube surfaces. When $\Delta J=0$ surfaces are inert, and when $\Delta J>0$ surfaces favour the metal hydride phase. We deliberately do not assign a particular physical length scale to lattice spacing, focusing instead on the correspondence between size-scaling trends in both simulation and experiment. We employed a Metropolis Monte Carlo procedure to mimic experimental protocols, and used umbrella sampling to calculate free energy barriers to nucleation ${ }^{26}$.

Decreasing nanocube size acts to favour the hydride phase, the more so as temperature is increased (Fig. 2). The midpoint 


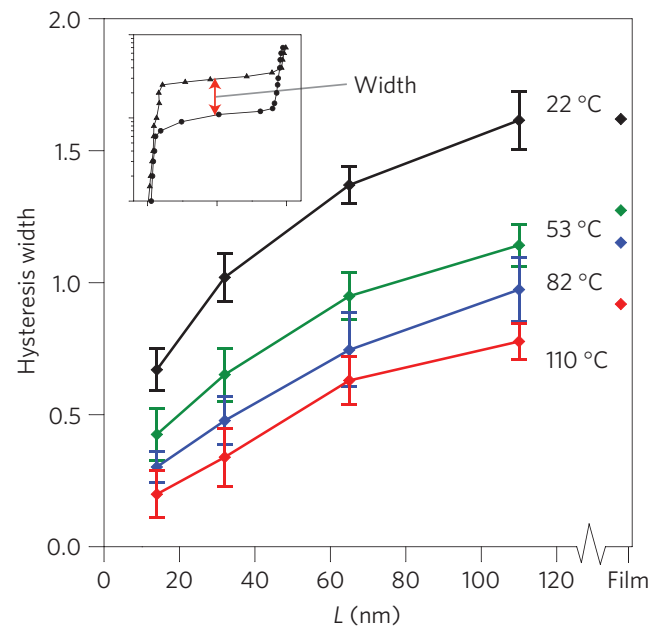

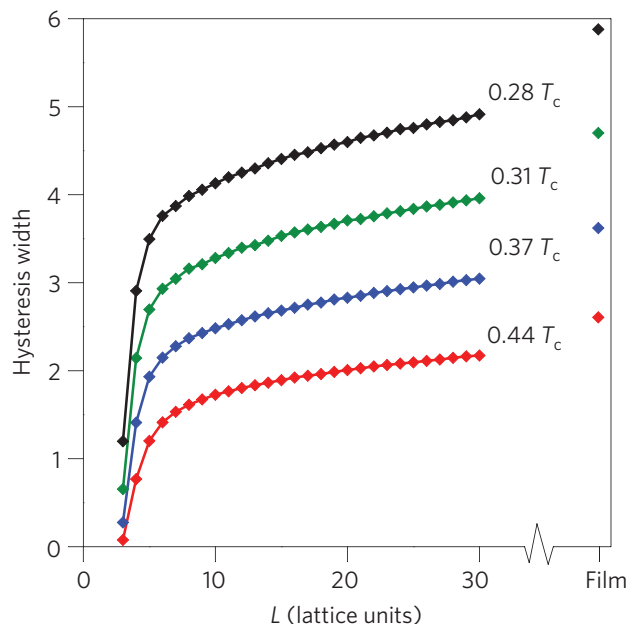

c

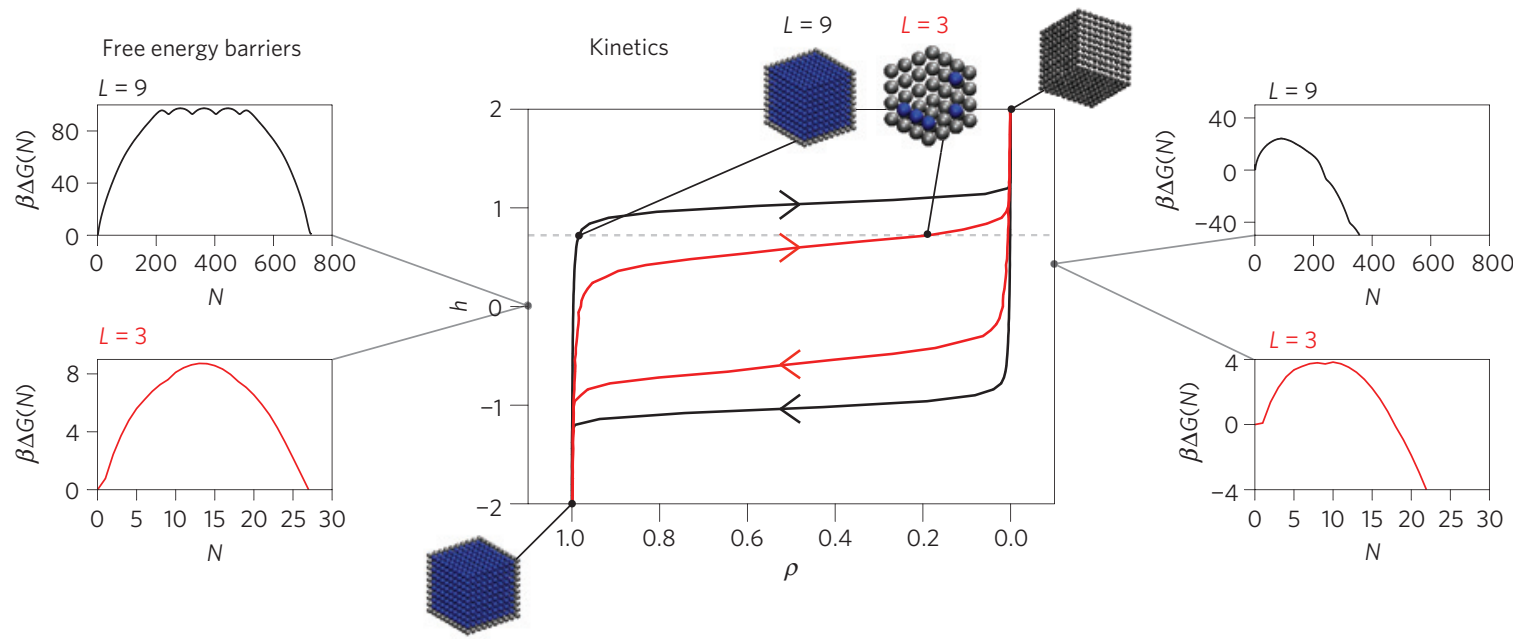

Figure 3 | Nanoconfined, thermally driven nucleation provides a natural explanation for the strong size dependence of nanocube kinetics. a,b, Hysteresis loop widths (vertical axis is $\ln \left(P_{\text {hydride }} / P_{\text {dehydride }}\right)$ ) in experiment (a) vary with temperature and nanocube size in a manner similar to hysteresis loop widths in Ising model simulations ( $\mathbf{b}$; vertical axis is $2\left(h_{\text {forward }}-h_{\text {reverse }}\right) / T$ ). This similarity suggests that size-dependent trends observed in experiment are a natural consequence of nanoconfined, thermally driven nucleation. Experimental error bars are calculated from 5 independent measurements. $\mathbf{c}$, To see this, consider Ising model magnetization-magnetic field hysteresis loops for small (red, $L=3$ ) and large (black, $L=9$ ) nanocubes (here we consider inert exteriors, $\Delta J=0$, for simplicity). Near the coexistence point $h=0$ there exists a free energy barrier to nucleation that frustrates phase change: one must wait for thermal fluctuations to surmount this barrier and generate a critical nucleus of the new phase. ( $N$ is the number of lattice sites in the nucleating cluster of the new phase). Crucially, this barrier is smaller in the smaller nanocube (see Fig. 4). As a result, phase transformation in the smaller cube occurs closer to the coexistence point $h=0$ (in both directions), and hysteresis is less pronounced than in the larger cube. Here $\rho$ is the scaled magnetization, as defined in the Methods.

$P_{\text {mid }}$ of the plateaux for forward and reverse transformations provides an estimate of the pressure $P_{\text {eq }}$ at which metal and metal hydride phases are equal in free energy (see Supplementary Information page 19-20, Figs S7, S8 and Table S2 for description of $P_{\text {mid }}$ calculation). As temperature increases, midpoint pressures increase, revealing that larger pressures must be applied to induce transformation to the hydride phase: the metal phase is stabilized. This stabilization can be seen by noting that the midpoints of the curves of Fig. 2a-bottom panel (high temperature) sit at larger pressures than their counterparts in Fig. 2a, top panel (low temperature). However, this trend is a characteristic of the bulk metal, and decreasing nanocube size acts to oppose it. These facts can be deduced by noting that midpoints of curves in Fig. 2a shift down as cube size is decreased (right to left). To demonstrate these trends clearly, we have constructed (Fig. 2b) a normalized Van't Hoff representation, by subtracting from our experimentally determined values of $\ln \left(P_{\text {mid }} / P_{0}\right)$ their bulk film counterparts. In this representation the bulk midpoint pressure lies, by construction, at a value of 0 . Values on the vertical axis below 0 therefore indicate a preference, relative to bulk, for the metal hydride phase. At fixed temperature we see clearly that smaller particles favour hydride formation (in agreement with the results of ref. 19). Furthermore, this preference becomes more pronounced as temperature increases: curves bend down as one looks right to left on Fig. 2b. Simulations (Fig. 2c) of Ising nanocubes reveal that such trends can be reproduced when nanocube exteriors prefer energetically the metal hydride phase $(\Delta J>0)$ to a degree that increases strongly with temperature. This determination is made straightforward by the intrinsic symmetry of the Ising model representation, and is concealed by the lattice gas one ${ }^{27,28}$ (Supplementary Section SIV).

Hysteresis loop behaviours can be reproduced by simulations that assume nanocube hydriding to be a thermally driven phase transition enclosed in a finite box, so providing a natural 


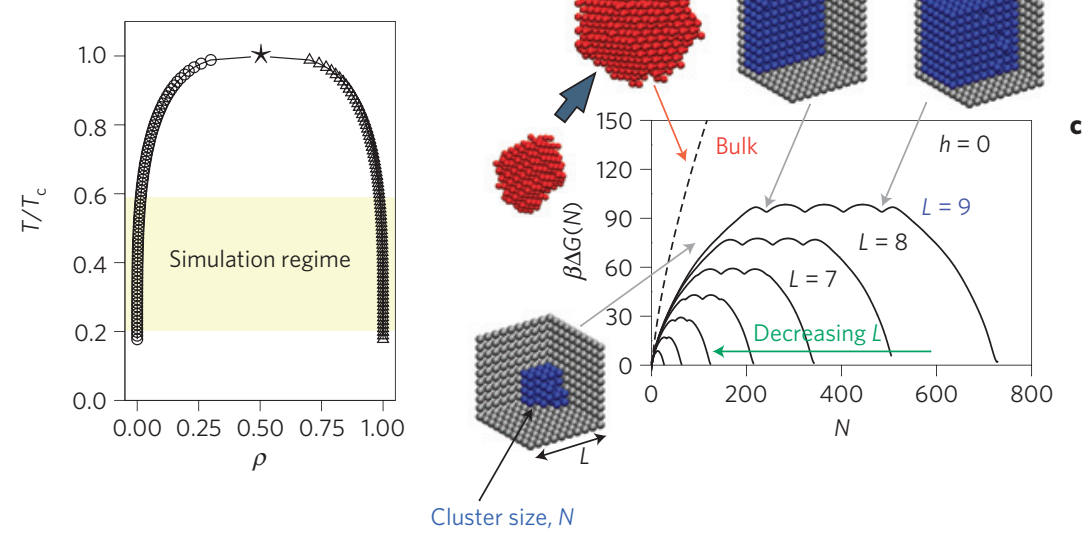

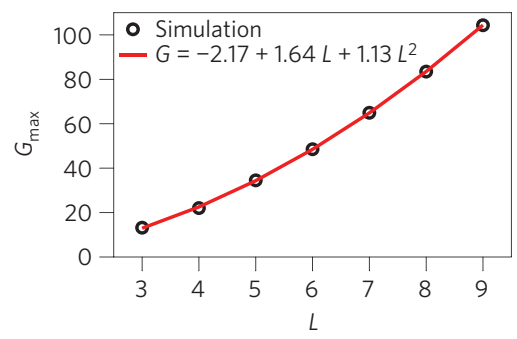

Figure 4 | Free energy barriers to phase change are controlled, near phase coexistence, by nanocube size. a, Phase diagram for the bulk Ising model obtained using Gibbs ensemble simulations. The simulation regime explored in this Article (shaded), appropriate to our experimental regime, is far from

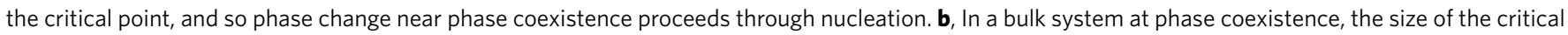

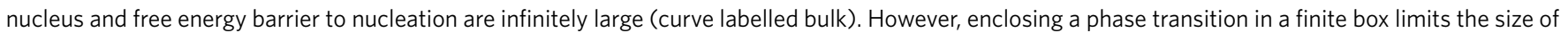

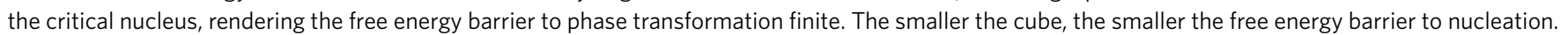
Consequently, less hysteresis is observed in dynamical simulations (see Fig. 3). c, Simulation reveals that nucleation barriers in nanocubes at phase coexistence scale as $c_{1} L+c_{2} L^{2}$ ( $c_{1}$ and $c_{2}$ are constants), where $L$ is cube side length. This scaling is consistent with a simple argument (Supplementary Section SIII) that assumes the critical nucleus is a two-dimensional film on an interior face of the nanocube.

explanation for the strong finite-size effects observed. In Fig. 2a we show hysteresis loops for nanocubes of different sizes. Hysteresis indicates that a system has fallen out of equilibrium on the timescale of measurement, and the extent of the hysteresis (here the width of the loop) provides information about the physical mechanism underlying the observed transformation. In Fig. 3a we show hysteresis loop widths as a function of nanocube size and temperature. It is evident, at all temperatures, that widths vary across the entire size range studied (up to $110 \mathrm{~nm}$ ), in striking contrast to earlier conclusions that nanocrystals become asymptotically bulk-like at about $15 \mathrm{~nm}$ (ref. 29). Ising model simulations (Fig. $3 \mathrm{~b}$ ) reproduce these trends qualitatively, providing a natural explanation for the strong finite-size effects observed: free energy barriers to nucleation are controlled by the size of the nanocube.

To explain this control, consider Fig. 3c. On driving a system (here the Ising model, by changing magnetic field) across a firstorder phase transition, one makes a previously unstable phase stable. However, the existing phase may be metastable, meaning that there exists a free energy barrier that must be surmounted (by thermal fluctuations) before the stable phase can be generated: this is the process of nucleation. The nucleation barrier governs the characteristic waiting time for phase transformation in a volume $V$ (and is roughly $\tau_{\text {wait }} \approx \tau_{\text {micro }}\left(V_{0} / V\right) \mathrm{e}^{\Delta E / k_{\mathrm{B}} T}$, where $\tau_{\text {micro }}$ is a characteristic microscopic attempt time, and $V_{0}$ is a microscopic volume). One must generally drive a system some distance past the transition (in forward and reverse directions) before $\tau_{\text {wait }}$ becomes of the order of the experimental waiting time, and transformation occurs. This distance, the hysteresis loop width, is governed by the size of the free energy barrier to nucleation.

The simulation regime explored in this work, appropriate to our experimental regime, is far from the critical point, and so phase change near phase coexistence proceeds through nucleation (Fig. 4a). In a bulk system, at a first-order phase transition, the size of the critical nucleus and the nucleation free energy barrier diverge. One must therefore drive a system well beyond the transition point to induce phase transformation, resulting in considerable hysteresis. However, a finite system (such as a nanocube) imposes a limit on how large a critical nucleus can be, so constraining the free energy barrier to nucleation to be finite ${ }^{30-32}$ and nanocube size dependent: free energy barriers to nucleation diminish as nanocubes are made smaller (Fig. 4b). Simple arguments (see Supplementary Section SIII) suggest that in a cube of side length $L$ the free energy barrier to nucleation scales as $\Delta E /\left(k_{\mathrm{B}} T\right) \propto c_{1} L+c_{2} L^{2}$ ( $c_{i}$ are constants) at a first-order phase transition; this scaling agrees qualitatively with that calculated from our simulations (Fig. 4c). As the nucleation barrier diminishes, so too does the distance between the phase transition and the location of phase transformation: consequently, hysteresis loop widths diminish as nanocubes are made smaller (Fig. 3c).

The strong correspondence between experiment and simulation (Fig. 3a,b) suggests that the observed size dependence of nanocube phase transformation kinetics is a natural consequence of nanoconfined, thermally driven nucleation: making cubes smaller makes free energy barriers to nucleation smaller, and therefore makes hysteresis loops narrower. In addition, increasing temperature reduces barrier heights relative to the thermal energy, so reducing hysteresis loop widths (Fig. 3a,b). We do not need to appeal to extrinsic physical mechanisms such as defects or stress ${ }^{11,23,33}$ to explain these trends. Indeed, were such effects present they could mask the scaling behaviour uncovered here, because the energy scales associated with extrinsic effects such as strain clamping can far exceed the thermal energy that drives intrinsic phase transformations.

Hydriding and dehydriding kinetics on sudden, large-pressure changes are enhanced as nanocrystal size decreases. Our luminescence-based approach allows us to monitor the real-time absorption/desorption kinetics of Pd nanocubes. Normalized luminescence response (Fig. 5a and Supplementary Fig. S11) was recorded following a sudden, large increase or decrease in hydrogen pressure, revealing a decrease in sorption timescale with decreasing nanocube size. Nanocubes also have faster kinetics than bulk Pd films (Supplementary Fig. S6). A microscopic insight into this behaviour is afforded by the Ising model, in which a similar hierarchy of sorption rates with system size is seen (Fig. 5b): both transformations are faster in smaller cubes. This enhancement arises because the new phase encroaches more easily at the sample surface than in the bulk, and because decreasing the size of a cube increases its surface-to-volume ratio. Starting from a pure phase, at zero magnetic field, the energy cost per bulk bond required to create 


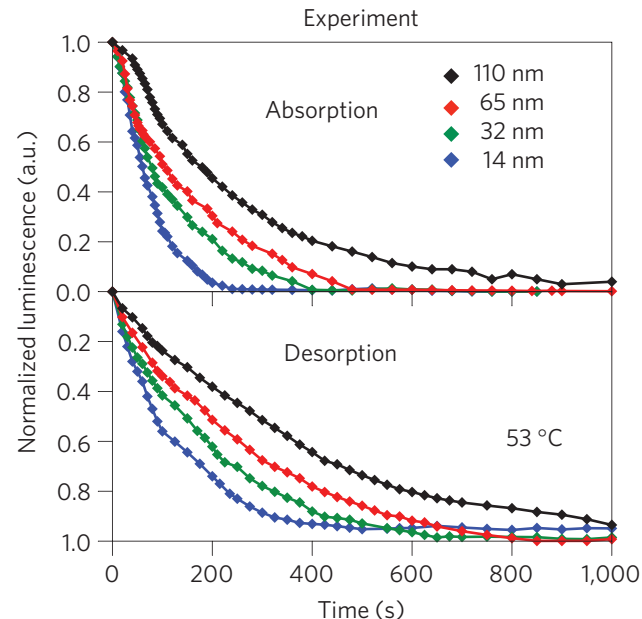

b

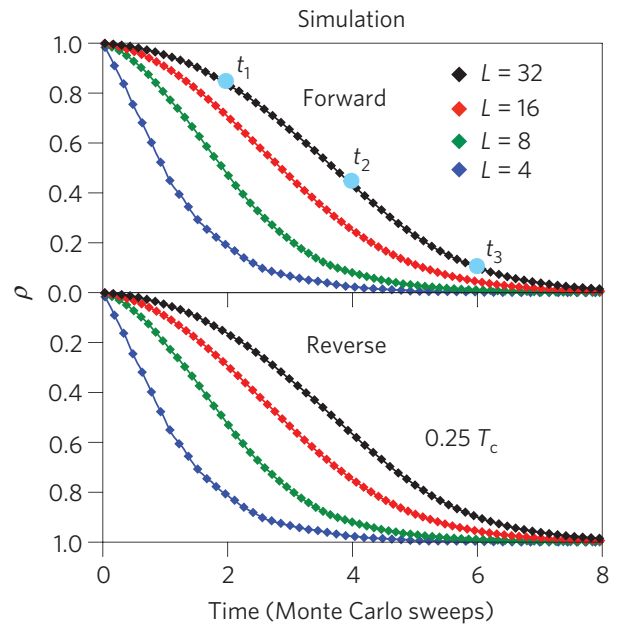

C
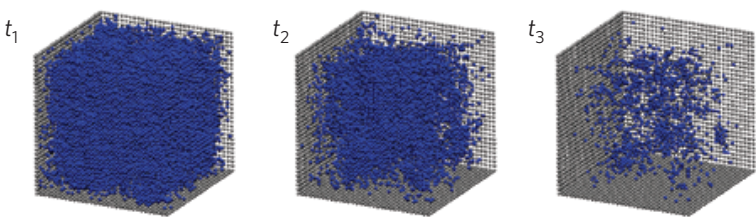

Figure 5 | Nanocube kinetics resulting from sudden, large pressure changes suggest that phase change begins at nanocube surfaces. a, Luminescence data obtained for $\mathrm{Pd}$ nanocubes at $53^{\circ} \mathrm{C}$ from experiments involving sudden pressure changes show enhanced kinetics of both hydriding and dehydriding transitions as nanocube size decreases. Data are normalized by mass of the nanocubes deposited. b, A similar hierarchy is seen in the Ising model (on sudden changes of magnetic field) whenever surface and bulk energy scales are such that phase change begins at nanocube surfaces (see snapshots (c)). c, In the snapshots, blue represents lattice cells that are in the metal phase and grey represents the periodic walls of the nanocube. If phase change begins instead in cube interiors, decreasing nanocube size leads to a slowing of kinetics (see Supplementary Fig. S16). The comparison between simulation and experiment thus suggests that both hydriding and dehydriding transformations begin at cube surfaces.

the new phase is $2 J$ (where $J$ is the bulk coupling). At the cube surface, however, the cost per bond is $\pm \Delta J$ (the sign depends on the direction of change). Thus, for a wide range of wall interactions, that is, $|\Delta J|<2 J$, both forward and reverse transformations are faster the smaller the cube. The enhancement of both adsorption and desorption kinetics seen in experiment thus suggests a preference for establishment of the new phase at the nanocube surface. Further, this similarity reveals that the Ising representation is a natural one for this system. The conventional lattice gas (particle-vacancy) representation of hydriding-dehydriding, when encased by an inert box ${ }^{23}$ (Supplementary Fig. S16), shows kinetic enhancement of one process as nanocube size decreases, but slowing of the reverse process (because one transformation begins at the cube surface, but the other begins in its interior). The lattice gas representation therefore misses important qualitative experimental trends, unless one deliberately mimics the Ising model representation by imposing a particle-wall attraction that is one-half the bulk energetic coupling ${ }^{23}$.

Activation energies for all systems derived from kinetic data (Supplementary Fig. S11) and extensive thermodynamic data including enthalpy, entropy and critical temperature of sorption derived from Van't Hoff plots (Supplementary Fig. S10) show a significant decrease in these parameters with decreasing nanocube size (Supplementary Table S1). These values clearly indicate size-dependent changes in hydriding thermodynamics and kinetics, identifying clear size-scaling relations for these thermally driven transformations supported by statistical mechanical simulations. This study suggests a general optimization strategy for design of hydrogen storage in metal hydride systems: tailor nanocrystal surface/volume ratio or surface energetics to generate a desired free energy barrier to phase transformation for given operational conditions.
In summary, this study is the first report of intrinsic size-scaling laws for hydriding transformations in nanocrystals. We have used a statistical mechanical model to show that the established physics of phase change in confinement explains data obtained by a new, direct metallic luminescence-based optical approach applied to size-controlled Pd nanocrystals. This approach enables direct correlations to be made between optical signals and the progress of the phase transition in the ensemble of nanocubes-which, by virtue of cubes' narrow size distributions, we expect to be representative of phase changes in individual cubes (Supplementary Figs S17 and S18)—thus providing a predictive framework for optimizing hydrogen storage density and kinetics in a variety of metal hydride systems under experimental conditions germane to storage applications, that is, well below the critical temperature (complementary analysis of phase transitions in systems near criticality is classically established $\left.{ }^{21,22,25,34-36}\right)$. We verified also that in the sub-critical (nucleation-dominated) regime relevant to our experiments, long-range hydrogen-hydrogen interactions, implicated in the behaviour of macroscopic Pd samples near the critical temperature $^{37}$, do not qualitatively change our conclusions (see Supplementary Section SV and Figs S20, S21 and S24).

We conclude that hydride nucleation in Pd nanocubes is driven by thermal fluctuations, not by sample strain or other extrinsic mechanisms. Our data reveal the profound effect of nanocrystal size on hydriding thermodynamics and kinetics ${ }^{38}$, for nanocrystals far larger than those previously thought to be bulk-like. This study immediately implies that pure size effects can be understood and productively employed over a much broader range of nanocrystal sizes than previously thought. Such control will impact fields in which controlling the thermodynamics of gas-nanocrystal interactions are paramount, ranging from gas capture and separations to catalysis, lithium-ion battery design, and photoelectrochemistry. 


\section{Methods}

Pd nanocube synthesis and characterization. Pd nanocubes were synthesized by modifying a previously published protocol ${ }^{39}$. The $14 \mathrm{~nm}$ nanocubes were synthesized as indicated previously ${ }^{39}$. The $32 \mathrm{~nm}$ nanocubes were synthesized by mixing $50 \mathrm{ml}$ of $50 \mathrm{mM}$ CTAB solution with $1.25 \mathrm{ml}$ of $10 \mathrm{mM} \mathrm{H}_{2} \mathrm{PdCl}_{4}$ solution and $1.5 \mathrm{ml}$ of the $14 \mathrm{~nm}$ nanocube suspension. Subsequently, $500 \mu \mathrm{l}$ of freshly prepared $100 \mathrm{mM}$ ascorbic acid was added, and the mixture was gently shaken, and left unperturbed at $60^{\circ} \mathrm{C}$ in a water bath for $2 \mathrm{~h}$. The $65 \mathrm{~nm}$ nanocubes and $110 \mathrm{~nm}$ faceted nanocrystals were synthesized similarly except only $200 \mu \mathrm{l}$ of the $14 \mathrm{~nm}$ nanocube suspension was added in the former and $100 \mu \mathrm{l}$ in the latter. The $14 \mathrm{~nm}$ nanocubes were centrifuged at $17,530 \mathrm{~g}$. for $30 \mathrm{~min} 5$ successive times to remove excess CTAB; the final isolated product was re-dispersed in $1 \mathrm{ml} \mathrm{H}_{2} \mathrm{O}$. The $32 \mathrm{~nm}$ nanocubes were centrifuged at $3,622 \mathrm{~g}, 65 \mathrm{~nm}$ nanocubes at $716 \mathrm{~g}$, and $110 \mathrm{~nm}$ nanocrystals at $548 \mathrm{~g}$, respectively, 3 times for $20 \mathrm{~min}$ each and finally re-dispersed in $1 \mathrm{ml} \mathrm{H}_{2} \mathrm{O}$.

Nanocubes were characterized by using a Zeiss Ultra55 field-emission scanning electron microscope. The extinction spectra were obtained on a UV3600 Shimadzu ultraviolet-visible/near-infrared spectrophotometer. XRD peaks were obtained using a Bruker D8 Discover X-ray diffractometer with a general area detector diffraction system using $\mathrm{Cu} \mathrm{K} \alpha$ radiation $(\lambda=0.154 \mathrm{~nm})$.

Substrate preparation for in situ studies. Pd nanocube suspensions were drop-cast on 2-mm-thick clean quartz windows (Thorlabs) and dried under vacuum at room temperature for $12 \mathrm{~h}$. The mass of the windows was taken before and after sample deposition to determine the mass of the nanocubes deposited. The mass of the nanocubes was determined to be $150 \pm 30 \mu \mathrm{g}$. Subsequent to drying, the nanocube films were gently rinsed with warm $18 \mathrm{~m} \Omega \mathrm{H}_{2} \mathrm{O}$, acetone and isopropanol to remove any residual surfactant. All nanocrystal films were then annealed for $2 \mathrm{~h}$ at $100{ }^{\circ} \mathrm{C}$ under vacuum to ensure a pristine surface. The $65 \mathrm{~nm}$ Pd films were deposited by electron beam evaporation $\left(10^{-6}\right.$ torr $)$ at $2 \AA \mathrm{s}^{-1}$ at room temperature, annealed for $2 \mathrm{~h}$ at $100^{\circ} \mathrm{C}$ and kept under vacuum.

Simulation model. We simulated the standard Ising model on a cubic lattice in three dimensions. On each of $N$ sites $i$ lives a spin variable $S_{i}$, which represents the metal hydride phase when it takes a value -1 (down), and the metal phase when it takes a value +1 ('up'). Sites interact according the Ising Hamiltonian

$$
H=-J \sum_{\langle i j\rangle} S_{i} S_{j}+h \sum_{i} S_{i}
$$

Here the first sum runs over all distinct pairs of nearest-neighbouring lattice sites, and the second runs over all sites. The parameter $J$ sets the bulk surface tension between phases, and determines how close the system is to the bulk critical temperature. The magnetic field $h$ is varied to drive the system from one phase to another; $2 h / T$ is analogous to the logarithm of experimental pressure. As a proxy for experimental luminescence we introduce the scaled magnetization $\rho \equiv 1 / 2\left(1+N^{-1} \sum_{i} S_{i}\right): \rho$ is 1 in the pure metal phase and 0 in the pure metal hydride phase. We considered bulk systems (large systems with periodic boundary conditions in all directions), and finite cubes of side $L$ enclosed by 6 walls (walls are built from spins that are unable to flip). Up and down spins adjacent to a wall interact with each nearest-neighbour wall spin with energies $J_{+}$and $J_{-}$, respectively; a non-zero difference $\Delta J \equiv J_{+}-J_{-}$means that the wall prefers one phase to the other. We performed simulations using standard single-particle Metropolis Monte Carlo dynamics and used standard umbrella sampling techniques ${ }^{26,40}$ to compute free energy barriers to nucleation.

Details of the in situ luminescence experiments and simulation protocol are provided in Supplementary Section SI.

\section{Received 23 March 2012; accepted 19 June 2013; published online 4 August 2013}

\section{References}

1. Sear, R. P. Nucleation: Theory and applications to protein solutions and colloidal suspensions. J. Phys. Condens. Matter 19, 033101 (2007).

2. Berube, V., Radtke, G., Dresselhaus, M. \& Chen, G. Size effects on the hydrogen storage properties of nanostructured metal hydrides: A review. Int. J. Energy Res. 31, 637-663 (2007).

3. Chen, C-C., Herhold, A. B., Johnson, C. S. \& Alivisatos, A. P. Size dependence of structural metastability in semiconductor nanocrystals. Science 276, 398-401 (1997).

4. Lee, B-S. et al. Observation of the role of subcritical nuclei in crystallization of a glassy solid. Science 326, 980-984 (2009).

5. Oxtoby, D. W. Nucleation of first-order phase transitions. Acc. Chem. Res. 31, 91-97 (1998).
6. Langhammer, C., Zhdanov, V. P., Zorić, I. \& Kasemo, B. Size-dependent kinetics of hydriding and dehydriding of Pd nanoparticles. Phys. Rev. Lett. 104, 135502 (2010).

7. Favier, F., Walter, E. C., Zach, M. P., Benter, T. \& Penne, R. M. Hydrogen sensors and switches from electrodeposited palladium mesowire arrays. Science 293, 2227-2231 (2001).

8. Jeon, K-J. et al. Air-stable magnesium nanocomposites provide rapid and high-capacity hydrogen storage without using heavy-metal catalysts. Nature Mater. 10, 286-290 (2011).

9. Bardhan, R., Ruminski, A. M., Brand, A. \& Urban, J. J. Magnesium nanocrystal-polymer composites: A new platform for designer hydrogen storage materials. Energy Environ. Sci. 4, 4882-4895 (2011).

10. Gremaud, R., Slaman, M., Schreuders, H., Dam, B. \& Griessen, R. An optical method to determine the thermodynamics of hydrogen absorption and desorption in metals. Appl. Phys. Lett. 91, 231916 (2007).

11. Baldi, A., Gonzalez-Silveira, M., Palmisano, V., Dam, B. \& Griessen, R. Destabilization of the $\mathrm{Mg}-\mathrm{H}$ system through elastic constraints. Phys. Rev. Lett. 102, 226102 (2009).

12. Langhammer, C., Larsson, E. M., Kasemo, B. \& Zorić, I. Indirect nanoplasmonic sensing: Ultrasensitive experimental platform for nanomaterials science and optical nanocalorimetry. Nano Lett. 10,3529-3538 (2010).

13. Langhammer, C., Zorić, I., Kasemo, B. \& Clemens, B. M. Hydrogen storage in Pd nanodisks characterized with a novel nanoplasmonic sensing scheme. Nano Lett. 7, 3122-3127 (2007).

14. Liu, N., Tang, M. L., Hentschel, M., Giessen, H. \& Alivisatos, A. P. Nanoantenna-enhanced gas sensing in a single tailored nanofocus. Nature Mater. 10, 631-636 (2011)

15. Varnavski, O. P., Mohamed, M. B., El-Sayed, M. A. \& Goodson, T. III Relative enhancement of ultrafast emission in gold nanorods. J. Phys. Chem. B 107, 3101-3104 (2003).

16. Fedorovich, R. D., Naumovets, A. G. \& Tomchuk, P. M. Electron and light emission from island metal films and generation of hot electrons in nanoparticles. Phys. Rep. 328, 73-179 (2000).

17. Luther, J. M., Jain, P. K., Ewers, T. \& Alivisatos, A. P. Localized surface plasmon resonances arising from free carriers in doped quantum dots. Nature Mater. 10, 361-366 (2011)

18. Eastman, D. E., Cashion, J. K. \& Switendick, A. C. Photoemission studies of energy levels in the palladium-hydrogen system. Phys. Rev. Lett. 27, 35-38 (1971).

19. Yamauchi, M., Kobayashi, H. \& Kitagawa, H. Hydrogen storage mediated by Pd and Pt nanoparticles. Chem. Phys. Chem. 10, 2566-2576 (2009).

20. Binney, J., Dowrick, N., Fisher, A. \& Newman, M. The Theory of Critical Phenomena (Oxford Univ. Press, 1992).

21. Alefeld, G. \& Völkl, J. Topics in Applied Physics: Hydrogen in Metals II: Application-Oriented Properties (Springer, 1978).

22. Buck, H. \& Alefeld, G. Hydrogen in palladium-silver in the neighbourhood of the critical point. Phys. Stat. Sol. B 49, 317-327 (1972).

23. Langhammer, C., Zhdanov, V. P., Zorić, I. \& Kasemo, B. Size-dependent hysteresis in the formation and decomposition of hydride in metal nanoparticles. Chem. Phys. Lett. 488, 62-66 (2010).

24. Salomons, E., Griessen, R., De Groot, D. G. \& Magerl, A. Surface tension and subsurface sites of metallic nanocrystals determined from $\mathrm{H}$-absorption. Europhys. Lett. 5, 449-454 (1988).

25. Wagner, H. \& Horner, H. Elastic interaction and the phase transition in coherent metal-hydrogen systems. Adv. Phys. 23, 587-637 (1974).

26. Ten Wolde, P. R., Ruiz-Montero, M. J. \& Frenkel, D. Numerical calculation of the rate of crystal nucleation in a Lennard-Jones system at moderate undercooling. J. Chem. Phys. 104, 9932-9947 (1996).

27. Zhdanov, V. P. \& Kasemo, B. The formation of a new phase in nanoparticles. Physica E 41, 775-778 (2009).

28. Zhdanov, V. P. \& Kasemo, B. Kinetics of the formation of a new phase in nanoparticles. Chem. Phys. Lett. 460, 158-161 (2008).

29. Pundt, A. \& Kirchheim, R. Hydrogen in metals: Microstructural aspects. Annu. Rev. Mater. Res. 36, 555-608 (2006).

30. Binder, K. \& Landau, D. P. Finite-size scaling at first-order phase transitions. Phys. Rev. B 30, 1477-1485 (1984).

31. Furukawa, H. \& Binder, K. Two-phase equilibria and nucleation barriers near a critical point. Phys. Rev. A 26, 556-566 (1982).

32. Rikvold, P. A., Tomita, H., Miyashita, S. \& Sides, S. W. Metastable lifetimes in a kinetic Ising model: Dependence on field and system size. Phys. Rev. E 49, 5080-5090 (1994).

33. Dura, J. A. et al. Porous $\mathrm{Mg}$ formation upon dehydrogenation of $\mathrm{MgH}_{2}$ thin films. J. Appl. Phys. 109, 093501 (2011).

34. Olsson, S. \& Hjörvarsson, B. Effect of biaxial elastic constraints on $\mathrm{H}-\mathrm{H}$ interactions in ultrathin vanadium. Phys. Rev. B 71, 035414 (2005).

35. Olsson, S., Blixt, A. M. \& Hjörvarsson, B. Mean-field-like structural phase transition of $\mathrm{H}$ in $\mathrm{Fe} / \mathrm{V}(001)$ superlattices. J. Phys. Condens. Matter 17, 2073-2084 (2005).

36. Pálsson, G. K. et al. Hydrogen site occupancy and strength of forces in nano-sized metal hydrides. Phys. Rev. B 85, 195407 (2012). 
37. De Ribaupierre, Y. \& Manchester, F. D. Experimental study of the critical-point behaviour of the hydrogen in palladium system. I. Lattice gas aspects. J. Phys. C 7, 2126-2139 (1974).

38. Jongh, P. E. d. \& Adelhel, P. Nanosizing and nanoconfinement: New strategies towards meeting hydrogen storage goals. Chem. Sus. Chem. 3, 1332-1348 (2010).

39. Niu, W., Zhang, L. \& Xu, G. Shape-controlled synthesis of single-crystalline palladium nanocrystals. ACS Nano 4, 1987-1996 (2010)

40. Hedges, L. O. \& Whitelam, S. Patterning a surface so as to speed nucleation from solution. Soft Matter 8, 8624-8635 (2012).

\section{Acknowledgements}

We would like to thank R. Hauge at Rice University, Houston, Texas for help with the optical cell. We would also like to acknowledge A. Schwartzberg, T. Mattox, R. Buonsanti, A. Ruminski, T. Kyukendall, I. Tamblyn and L. Maibaum for helpful discussions. Work at the Molecular Foundry was supported by the Office of Science, Office of Basic Energy Sciences, of the US Department of Energy under Contract No. DE-AC02-05CH11231. J.J.U. and R.B. are supported under the US Department of Energy Hydrogen Storage Program, B\&R code KC0202020. C.L.P. and A.J. are supported under Mohr Davidow
Ventures and Berkeley Sensor and Actuators Center. L.O.H. was supported by the Center for Nanoscale Control of Geologic $\mathrm{CO}_{2}$, a US D.O.E. Energy Frontier Research Center under Contract No. DE-AC02-05CH11231.

\section{Author contributions}

J.J.U. and R.B. conceived and designed the experiments. R.B. synthesized and characterized the nanoparticles and performed the experiments. C.L.P. designed and built the optical cell; C.L.P. and R.B. built the high-vacuum gas-flow system. L.O.H. and S.W. conceived the simulation protocol, and L.O.H. carried out the simulations. R.B., L.O.H., S.W. and J.J.U. analysed the data and wrote the manuscript. A.J., S.W. and J.J.U. discussed the results and commented on the manuscript.

\section{Additional information}

Supplementary information is available in the online version of the paper. Reprints and permissions information is available online at www.nature.com/reprints. Correspondence and requests for materials should be addressed to S.W. or J.J.U.

\section{Competing financial interests}

The authors declare no competing financial interests. 


\section{Uncovering the intrinsic size dependence of hydriding phase transformations in nanocrystals}

Rizia Bardhan ${ }^{1, \xi, \ddagger}$, Lester O. Hedges ${ }^{1, \xi}$, Cary L. Pint ${ }^{2,3, \dagger}$, Ali Javey ${ }^{2,3}$, Stephen Whitelam ${ }^{1 *}$, Jeffrey J. Urban $^{1 *}$

${ }^{1}$ Molecular Foundry, Materials Sciences Division, Lawrence Berkeley National Laboratory, Berkeley, CA 94720, USA,

${ }^{2}$ Department of Electrical Engineering and Computer Sciences, University of California, Berkeley, CA

94720, USA,

${ }^{3}$ Berkeley Sensor and Actuator Center, University of California, Berkeley, CA 94720, USA

$\$$ Current address: Department of Chemical and Biomolecular Engineering, Vanderbilt University,

Nashville, TN 37235

$\dagger$ Current address: Department of Mechanical Engineering, Vanderbilt University, Nashville, TN 37235

$\xi$ These authors have contributed equally to this work

* To whom correspondence should be addressed. E-mail: swhitelam@lbl.gov and jjurban@lbl.gov

\section{List of Contents:}

I. Methods
A. In situ luminescence experiments
B. Simulation protocol

II. Detailed discussion of $\mathrm{Pd}$ luminescence and $\mathrm{H}_{2}$ sensing.
A. Pd luminescence and quantum yield of nanocubes
B. Pd luminescence change during $\mathrm{H}_{2}$ sorption.
C. Sensitivity of luminescence-based approach with size and particle density.
D. Scaling between luminescence signal and hydrogen concentration.
E. Surface area calculation for nanocrystals with different shapes.

III. Scaling argument for nucleation in nanocrystals.

IV. Note on Ising- and lattice gas representations of hydriding phase transformations.

V. Effect of long-range hydrogen-hydrogen interactions

Table S1: Enthalpy, entropy, critical temperature, and activation energy of hydrogen sorption.

Table S2: $\mathrm{P}_{\text {hyd }}, \mathrm{P}_{\text {dehyd }}, \mathrm{P}_{\text {mid }}$ and $\operatorname{Ln}\left(\mathrm{P}_{\text {mid }}\right)$ values evaluated at luminescence of 0.5 .

Table S3: Strain calculated from XRD peaks for Pd film and Pd nanocubes before and after hydriding.

Figure S1: Photoluminescence (PL) emission of Pd nanocubes of different sizes compared to reference sample of Rhodamine 6G (R6G). Corresponding quantum yield (QY) values are shown in inset.

Figure S2: TEM and electron diffraction before and after hydriding and dehydriding transitions, and XRD, and extinction spectra of Pd nanocubes.

Figure S3: TEM micrographs and luminescence spectra of $3 \mathrm{~nm}$ and $7 \mathrm{~nm}$ Pd nanocrystals. 
Figure S4: Schematic representation of optical setup.

Figure S5: Luminescence spectra of Pd nanocubes of different sizes.

Figure S6: Thermodynamic and kinetic data of $\mathrm{Pd}$ film.

Figure S7: P-L isotherms at $53{ }^{\circ} \mathrm{C}$ and $110{ }^{\circ} \mathrm{C}$.

Figure S8: Modified Van't Hoff plots derived by two different methods.

Figure S9: Sieverts Analysis of Pd nanocubes

Figure S10: Van't Hoff plots of Pd nanocubes at four different temperatures.

Figure S11: Additional kinetic data of Pd nanocubes at $22{ }^{\circ} \mathrm{C}, 82{ }^{\circ} \mathrm{C}, 110^{\circ} \mathrm{C}$.

Figure S12: Arrhenius rate law plots of $\mathrm{Pd}$ nanocubes from kinetic data.

Figure S13: XRD before and after hydriding Pd nanocubes.

Figure S14: Control study of luminescence from Pd vs. the quartz substrate.

Figure S15: Control study of hydriding quartz substrate, CTAB and Pd nanocubes under $\mathrm{N}_{2}$ flow.

Figure S16: Artifacts of the lattice gas representation.

Figure S17: Ensemble averaging and polydispersity.

Figure S18: P-L isotherm of polydisperse Pd sample and corresponding SEM image.

Figure S19: P-L isotherm magnified to clearly represent the trends in $\mathrm{P}_{\text {mid }}$ with increasing nanocube size.

Figure S20: The scaling of hysteresis loop width with temperature and nanocube size as seen in the Ising model and with the addition of a long-ranged elastic interaction.

Figure S21: Simulations showing free energy barrier heights as a function of nanocube size as seen in the Ising model at phase coexistence and with the addition of a long-ranged elastic interaction

Figure S22: XRD peaks before and after hydriding of Pd film compared with Pd nanocubes for strain calculation.

Figure S23. The elastic strain energy calculated from XRD data at $2 \theta=38.9$ and 45.4 is shown as a function nanocube size.

Figure S24. Simulations showing local and mean-field interactions to capture the physics appropriate to our experimental regime.

\section{Methods}

\section{A. In-situ luminescence experiments}

The pristine nanocrystal films and bulk Pd film were transferred from the vacuum oven to a custom-built stainless steel gas-tight cell with optical windows and connected to a high vacuum pump (Varian Inc.). Sample temperatures were controlled by inserting cartridge heaters (McMaster Carr Inc.) into slots in the optical cell and connecting the cartridge heaters to a Variac transformer (Variac Inc.) for controlling the heating rate. Temperature was monitored with a temperature controller fitted with a thermocouple (Omega Inc.). The thermocouple was inserted into the optical cell and kept in close contact with the substrates. The cell was evacuated for $2 \mathrm{hrs}$ at room temperature and then the samples were activated by repeated cycles of hydrogen purging and evacuation. Clean surfaces are essential to ensure the observed 
optical response is due to hydrogen absorption and desorption instead of surface contamination or adsorbate effects. Raman spectroscopy showed no peaks from CTAB after cleaning the surface, and only Pd luminescence was observed (Fig.1e-f, Fig. S5). After the activation step, the cell was evacuated for 3 hrs and the pressure of the cell was adjusted to $<1$ mbar. At such low pressure, the H/Pd ratio is less than 0.005. The luminescence spectra were obtained on a confocal Raman microscope (WiTec alpha300). All samples were excited with a $532 \mathrm{~nm}$ diode laser at $5 \mathrm{~mW}$ power with a $10 \mathrm{X} / 0.25 \mathrm{NA}$ objective lens $(\sim 2.5$ $\mu \mathrm{m}$ spot-size) and 5 second integration time. The gas flow was held constant at $150 \mathrm{sccm} / \mathrm{min}$, controlled with a mass flow controller (MFC, Sierra Instruments) during the experiment, and the pressure of the cell was then manually increased from $<1$ mbar to $800-900$ mbar in small steps with the low-flow metering valve (Swagelok Inc.). The pressure of the cell was monitored with a digital pressure transducer (MKS Instruments). The samples were equilibrated at each pressure and luminescence was subsequently recorded. After complete hydriding of the nanocrystals, the experiment was reversed. The gas flow was set to $0 \mathrm{sccm} / \mathrm{min}$ on the MFC and the pressure was manually decreased to $\sim 1 \mathrm{mbar}$ in small steps; the samples were equilibrated at each pressure. The kinetic experiments were executed similarly-during hydriding, the gas flow was held constant at $150 \mathrm{sccm} / \mathrm{min}$ and the pressure of the cell was instantaneously increased to 900 mbar. The plateau pressure of absorption is significantly lower than 900 mbar for all the samples at the temperature range examined in these experiments. After the luminescence signal stabilizes, the experiment is reversed by setting the gas flow to $0 \mathrm{sccm} / \mathrm{min}$ and instantly evacuating the optical cell to $\sim 1$ mbar to proceed with hydrogen desorption. All luminescence profiles were normalized by mass of the Pd nanocubes deposited.

\section{B. Simulation protocol:}

Simulations were carried out using standard single spin-flip Metropolis Monte Carlo (MC): a site was chosen at random; the spin was inverted; and the move was accepted with 
probability $\min \left(1, \exp \left(-\Delta E / k_{B} T\right)\right)$, where $\Delta E$ is the energy change associated with the trial move. Spin flips represent a change of the underlying lattice from the metal to the metal hydride conformation (or vice versa), rather than diffusion of hydrogen throughout the sample. We argue that the latter timescale is much faster than the timescale for phase transformation. The diffusion constant $D$ of hydrogen in palladium metal is of order $10^{-11} \mathrm{~m}^{2} / \mathrm{s}^{1}$. Hence the characteristic time $R^{2} / D$ for a hydrogen molecule to diffuse the side length $R$ of a $100 \mathrm{~nm}$ cube is of order $10^{-3} \mathrm{sec}$. This time is much less that the seconds or minutes on which pressure changes were exerted and phase transformations observed; we therefore assume in simulations that magnetic field (equivalent to the logarithm of hydrogen pressure) is constant throughout the simulation box, and that spin flips correspond to changes of lattice composition.

Hysteresis simulations (Fig. 3, main text) were performed by starting from the + phase and cycling the magnetic field from -3 to 3 and back again. This change of magnetic field was sufficiently large to drive the system from a pure + phase to a pure - phase at all conditions considered. The forward and reverse stage of each sweep ( -3 to 3 , or 3 to -3 ) was 1,000 MC sweeps in length (a sweep equals $N$ trial moves, where $N$ is the number of sites in the bulk of the simulation box) with the magnetic field changed in 100 linearly spaced intervals. In order to improve statistics the simulations were repeated at least 100 times for each data point (more repetitions were used for smaller box sizes). Although the size and shape of a hysteresis loop is weakly dependent on the time scale over which the magnetic field is cycled (hysteresis is larger for faster cycling) the qualitative nature of our results is not sensitive to cycling rate. The simulation time scale was thus chosen for computational convenience.

Free energy barriers (Fig.4) were computed using umbrella sampling with hard wall constraints on the size of the largest cluster in the simulation box. Different sampling windows were stitched together using the multiple histogram method. The curves in panels a) and b) have been shifted such that $\Delta G=G(N)-G(1)$. 
Kinetic simulations (Fig. 5) were performed starting from either the + or - phase and then instantaneously changing the magnetic field to +3 or -3 respectively. Once again, although the magnitude of the magnetization quench changes the rate at which transformations occur it does not change the qualitative behavior observed.

\section{Detailed discussion of Pd luminescence and $\mathrm{H}_{2}$ sensing}

\section{A. Pd luminescence and Quantum Yield of Nanocubes}

Optically-excited luminescence from nanoscale noble metals have been observed for Au, Ag, and $\mathrm{Cu}$, and is attributed to a combination of several processes including radiative decay of surface plasmons, local-field enhancements specifically in clustered-nanoparticle films due to the presence of "hot-spots" and inter- and intra-band excitations of surface plasmons. ${ }^{2-4}$ As shown in Fig. S2F, the Pd nanocubes support plasmon resonances in the UV-visible region. The observed luminescence from $\mathrm{Pd}$ nanocubes thus results from the surface plasmon dipole emission after direct plasmon excitation by visible light. Followed by excitation of the surface plasmon coherent electronic motion, relaxation of these electronic motions results in the radiative recombination of the sp-electrons with holes in the d-band which gives rise to the observed emission. ${ }^{3}$

The PL quantum yields of Pd nanocubes dispersed in aqueous media were determined by comparing their luminescence emission with a reference sample of rhodamine $6 \mathrm{G}$ solution with known quantum yield of $90 \%{ }^{5}$ Rhodamine $6 \mathrm{G}$ was chosen as its spectral profile most closely matches that of the Pd nanocubes out of all available QY standardization dyes. The luminescence emission of a sample may be expressed as

$$
F=k \Phi c \alpha l P
$$


where $\mathrm{F}$ is the integrated luminescence signal, $k$ is a parameter constant of the instrument, $\Phi$ is the luminescence quantum yield of the sample, $c$ is concentration of the sample (or fluorophore number density), $\alpha$ is the one-photon absorption cross section, $l$ is the photon absorption path length, $P$ is the incident flux of photons (or laser power). By measuring the luminescence spectra (Figure $\mathrm{S} 1$ ) $F_{\mathrm{S}}$ (nanocube sample) and $F_{R}$ (rhodamine $6 \mathrm{G}$ reference) corresponding to the emission regions were obtained. The luminescence spectra were recorded on a confocal Raman microscope (WiTec alpha300). All samples were excited with a $532 \mathrm{~nm}$ diode laser using a 40X/0.5 NA objective lens and data was acquired at 5 second integration time under similar conditions. This implies that $k$ and $l$ are the same for all nanocube samples and the reference sample.

Hence the quantum yield (QY) of the nanocubes can be determined by comparing $F s$ and $F_{R}$ for the nanocube samples and reference sample as follows,

$$
\Phi_{S}=\frac{F_{S}}{c_{S} \alpha_{S} P_{S}} \frac{c_{R} \alpha_{R} P_{R}}{F_{R}} \Phi_{R}
$$

Since $c \alpha$ represents the absorption coefficient $(A)$ of the sample at the excitation wavelength (BeerLambert law), the term of $c_{R} \alpha_{R} / c_{S} \alpha_{S}$ can be replaced by $A_{R} / A_{S}$. Equation 2 can then be simplified as follows,

$$
\Phi_{S}=\frac{F_{S}}{A_{S} P_{S}} \frac{A_{R} P_{R}}{F_{R}} \Phi_{R}
$$

where the absorption coefficients of the reference sample and nanocube samples were measured using a UV3600 Shimadzu UV-vis/near-IR spectrophotometer. Quantum yields $\left(\Phi_{\mathrm{S}}\right)$ of nanocube samples were then determined by following equation 3 and are shown in the inset in Figure S1.

\section{B. Pd luminescence change during $\mathrm{H}_{2}$ sorption}


The change in luminescence during hydrogen absorption and desorption may be understood by examining the density of states (DOS) of Pd and PdH. Calculated DOS of Pd and PdH have shown new set of states for $\mathrm{PdH}$ below the bottom of the $\mathrm{Pd} d$ bands formed due to the strong interaction of the hydrogen $1 \mathrm{~s}$ electron states and the low-lying Pd states. ${ }^{6,7}$ These new states show strong hybridized hydrogen-palladium bonding character but are decreased in magnitude compared to Pd DOS due to sharing of the hydrogen by four palladiums; i.e. in a cubic unit cell containing four palladiums in the original face-centered cubic positions with a single hydrogen in one of the octahedral site. ${ }^{6}$ This decrease in density of states as Pd transforms to the hydride phase is expected to change the plasmon resonance diminishing the sp-electron and d-hole recombination, thereby decreasing the luminescence. The phenomenon is reversed during dehydriding; $\mathrm{PdH}_{\mathrm{x}}$ decomposes to $\mathrm{Pd}$ increasing the $\mathrm{DOS}$ of $\mathrm{Pd}$ which enhances the electron-hole recombination and subsequently increases the radiative decay of surface plasmons resulting in regaining of the luminescence.

In addition to $\mathrm{H}_{2}$ sensing, this luminescence-based technique can be generalized for sensing other gaseous molecules such as $\mathrm{CO}$ and NO which are known to adsorb on Pd surfaces as well. ${ }^{8}$ Besides noble metals, this luminescence-based approach can also be extended to other nanocrystal systems ${ }^{9}$ to understand interactions at interfaces (solid/gas, or solid/liquid) enabling a wide-range of applications by simply varying the wavelength of excitation.

\section{Sensitivity of luminescence-based approach with size and particle density}

Typically for optical characterization of hydriding/dehydriding behavior in Pd nanocrystals $<10$ nm, gold nanoantennas are coupled to the Pd crystals for signal enhancement owing to the weak plasmon resonances of small Pd clusters. ${ }^{10,11}$ However, with this luminescence-based approach, a systematic sizeand shape-dependent study can be obtained for Pd nanocrystals in the size range of sizes $3-110 \mathrm{~nm}$ 
without the need of plasmonic oscillators for signal enhancement. Spherical Pd nanocrystals of $3 \mathrm{~nm}$ and $7 \mathrm{~nm}$ in diameter also show strong luminescence (Figure S3). Pd typically adopts a spherical shape for sizes $<10 \mathrm{~nm}$; spherical Pd nanocrystals are bound by [111] planes $^{12}$ compared to the cubic nanocrystals which are bound by [100] planes (Figure S2). The geometry, electronic structure, surface reactivity and energetics of the different crystal facets of $\mathrm{Pd}((100),(111)$ and (110)) differ from each other attributed to the different orbital occupations and bond populations of surface Pd atoms. This determines variations in energies of hydrogen dissociation on the surface, penetration into subsurface sites and bulk diffusion. A detailed discussion of the energetics of $\mathrm{H}$ near different Pd crystal facets can be found elsewhere. ${ }^{13,14}$ Owing to these differences the changes in luminescence during hydriding/dehydriding of spherical Pd nanoparticles have not been compared with the cubic Pd nanocrystals to avoid any ambiguities. A detailed study utilizing this luminescence approach for hydriding/dehydriding behavior in the smaller size-regime is currently underway. Additionally, shape-effects play a critical role for nanoparticle sizes $\leq$ $15 \mathrm{~nm}$ in terms of the available surface area for $\mathrm{H}_{2}$ sorption (see section $\mathrm{E}$ ). Such shape-effects have been shown to play a significant role in other applications as well such as catalysis. The catalytic activity of nanocrystals are altered owing to the percentage of edge and corner atoms in the different shapes that determines the rate of catalysis. ${ }^{15}$

The luminescence-based approach is also highly sensitive; photoluminescence was observed even from a sub-monolayer of nanocrystals at $2 \mathrm{~mW}$ laser power. Therefore the luminescence-based approach is highly useful for measuring energetics of gas sensing in ensembles of nanocrystals over a wide sizerange. Although for single particle measurements plasmonic antennas may be necessary for enhancing the signal.

\section{Scaling between luminescence signal and hydrogen concentration}


Zorić et al. have recently demonstrated that the change in optical signal of Pd nano- and microstructures during hydriding and dehydriding can be linearly correlated to the hydrogen concentration stored in $\mathrm{Pd}$. The hydrogen concentration was obtained gravimetrically by using a quartz crystal microbalance. ${ }^{16}$ Enthalpy and entropy of hydrogen absorption and desorption obtained optically and gravimetrically overlapped quiet well demonstrating that alterations in optical signature during hydrogen sorption can be directly correlated to H/Pd ratios. Experiments correlating Pd luminescence to hydrogen concentration obtained gravimetrically is currently underway.

\section{E. Surface area: volume calculations for nanocrystals of varying shapes:}

Although the largest nanocrystals $(110 \mathrm{~nm})$ were cuboctahedral instead of perfect cubes (Figure 1), the effect of varying nanocrystal shape on differential surface area: volume ratio should be demonstrably minimal for nanocrystals of diameter $>100 \mathrm{~nm}$. This allows us to directly compare hydriding and dehydriding thermodynamics and kinetics between nanocubes and the larger, faceted crystals. However if nanocrystals were $\leq 15 \mathrm{~nm}$, shape effects could play a critical role. To quantify this, we calculate the surface areas of a sphere, cube and octahedron with fixed volumes.

Cube: Side length $=\mathrm{a}=14 \mathrm{~nm}$, Volume $=\mathrm{a}^{3}=2.744 \times 10^{3} \mathrm{~nm}^{3}$, Surface Area $=6 \mathrm{a}^{2}=1.176 \times 10^{3} \mathrm{~nm}^{2}$.

Sphere: Volume $=4 / 3 \pi \mathrm{a}^{3}=2.744 \times 10^{3} \mathrm{~nm}^{3}$, then radius $=\mathrm{a}=8.685 \mathrm{~nm}$. Surface Area $=4 \pi \mathrm{a}^{2}=0.9478$ $\mathrm{x} 10^{3} \mathrm{~nm}^{2}$.

Octahedron: Volume $=1 / 3\left(\sqrt{ } 2 \mathrm{a}^{3}\right)=2.744 \times 10^{3} \mathrm{~nm}^{3}$, then side length $=\mathrm{a}=17.988 \mathrm{~nm}$. Surface Area $=$ $2 \sqrt{ } 3 \mathrm{a}^{2}=1.121 \times 10^{3} \mathrm{~nm}^{2}$.

These calculations show cubes of $14 \mathrm{~nm}$ edge length have $\sim 25 \%$ larger surface area than spheres of equivalent volume and $\sim 5 \%$ larger surface area than octahedrons of equivalent volume. Cubes of even larger edge length are expected to have even smaller differences in surface areas compared to those of 
varying shape, such as spheres and octahedrons, thus minimizing any surface area contribution to the behavior observed at $110 \mathrm{~nm}$.

\section{Scaling argument for nucleation in nanocrystals}

Inspection of umbrella sampling configurations (Fig. 4a, main text) suggests that we can roughly approximate the nucleus within a nanocube as a square film of side $x$ growing from the corner of the cube. The free energy required to grow this film is, in a classical scaling approximation (see e.g. Ref. ${ }^{17}$ ).,

$$
\Delta E(x)=2 x(\sigma+\Delta J)+x^{2}(\sigma+\Delta J-2 h)
$$

where $\sigma$ is the bulk surface tension, $\Delta$ is the preference of the wall for one phase over the other, and $h$ is magnetic field. For small $h$ (i.e. at or near coexistence), this barrier is largest when the film covers one face of the cube, i.e. when $x=L$, and is $\Delta E=\Delta E(L)=\mathrm{c}_{1} L+\mathrm{c}_{2} L^{2}$ (see Fig. $4 \mathrm{~b}$ ). Hence we would expect to see nucleation happen in a time (see main text) that scales to leading order in $L$ as $\tau_{\text {wait }} \propto L^{-3} \exp \left(\mathrm{c}_{1} L+\mathrm{c}_{2} L^{2}\right)$ (in units such that $k_{\mathrm{B}} T=1$ ). The prefactor $L^{-3}$ reflects the fact that, in the absence of other factors, the rate of nucleation should increase with system size. However, in contrast to bulk systems, here the system size also dictates the free energy barrier for nucleation, and the resulting term $\exp \left(\mathrm{c}_{2} L^{2}\right)$ dominates. At coexistence $(h=0)$, and in the bulk limit $L \rightarrow \infty$, the timescale for nucleation diverges, as expected. Far enough away from coexistence, and for large system sizes, the free energy barrier $\Delta E(x)$ takes its maximum for $x=x_{0}<L$. In this case the barrier height is $\Delta E=\Delta E\left(x_{0}\right)=(\Delta J+\sigma)^{2} /(2 h-\Delta J-\sigma)$, which is independent of $L$, and the timescale for nucleation goes as $\tau_{\text {wait }} \propto L^{-3}$.

\section{Note on Ising- and lattice gas representations of hydriding}


The dual enhancement in sorption rate with decreasing particle size seen in experiment arises naturally in the Ising model representation (Fig 5): both magnetization and demagnetization transformations are faster for smaller box sizes. This dual enhancement holds even when the walls have no energetic preference for one phase over the other. This is not true in the lattice gas representation (occupancies $n_{i}=\frac{1}{2}\left(1+S_{i}\right)$, where the $S_{i}= \pm 1$ are Ising spins), if one dictates that walls are energetically inert: in this representation, the particle phase $\left(n_{i}=1\right)$ experiences an unfavorable surface tension with the wall, but the vacancy phase $\left(n_{i}=0\right)$ does not. Some artifacts arising from this disparity are illustrated in Fig. S16. Notably, the critical point is shifted away from zero magnetic field, making it hard to cleanly deduce the effect of the surface interaction with each phase. In addition, one transformation is speeded, while the other is slowed. To eliminate this disparity, one must make walls attract particles with one-half of the particle-particle interaction energy, effectively mimicking the Ising representation. We argue that it is more natural to retain the symmetry intrinsic to the Ising model in order to model the experiments described in the main text.

\section{Effect of long-range hydrogen-hydrogen interactions}

Studies done on macroscopic palladium samples ${ }^{18}$ show that long-range hydrogen-hydrogen interactions, mediated by the elasticity of the host nanocrystal, dominate a sample's behavior near the critical temperature, resulting in mean-field critical exponents. However, for Pd nanocrystals we find that short-range interactions alone are sufficient to capture experimental trends of kinetics observed well below the critical temperature (Figs. 2-5). In Figs. S20 and S21 we demonstrate this fact by reporting the results of a series of simulations in which we added to our Ising model Hamiltonian the long-range elastic term 


$$
H_{\mathrm{mf}}=-\left(J_{\text {elastic }} / N\right) \sum_{i j} S_{i} S_{j}
$$

where (for concreteness) $J$ elastic $=J$. Here the sum runs over all $N$ lattice sites for which $i \neq j$, i.e. all sites interact with all other sites. Up to an unimportant constant term this elastic energy can be written $H_{\mathrm{mf}}=-\left(J_{\text {elastic }} / N\right) m^{2}$, where $m$ is the system's magnetization. The model, so modified, no longer falls in the Ising universality class. However, for nanocrystals in the nucleation regime, the mean-field term acts only as a perturbation. As shown in Figs S20 and S21, the size-scaling behavior observed in the absence of the long-range term is preserved. Our conclusions are therefore unaffected by the presence or absence of such a term.

The physical reason for this robustness is that kinetics observed in our study are driven largely by the surface tension between metal and metal hydride phases: sluggish nucleation kinetics result from a free energy barrier generated by an unfavorable surface tension between old and new phases (Figs. 2-4); and the non-nucleation kinetics seen in Fig. 5 also result from the presence of a surface tension (or else phase change would proceed anywhere in the system). Surface tension is generated by spatially varying interactions (here nearest-neighbor interactions), and the mean-field term acts only a perturbation to this surface tension. This finding is in agreement with ref. 10, which draws on the results of ref. 19 to demonstrate the importance of surface tension and short-range interactions to Pd nanocrystal kinetics below the critical temperature. 


\section{Supplementary Figures}

Table S1. Hydrogen absorption $(\alpha-\beta)$ and desorption $(\beta-\alpha)$ enthalpy $(\Delta \mathrm{H})$, entropy $(\Delta \mathrm{S})$, critical temperature $\left(\mathrm{T}_{\mathrm{C}}\right)$ and activation energy $\left(\mathrm{E}_{\mathrm{A}}\right)$ of $\mathrm{Pd}$ nanocubes and $\mathrm{Pd}$ film, as well as bulk $\mathrm{Pd}$ from the literature.

\begin{tabular}{|c|c|c|c|c|c|c|c|c|}
\hline \multicolumn{6}{|c|}{ Hydriding-dehydriding transformation data } & \multicolumn{3}{|c|}{ Activation energies } \\
\hline Size & $\begin{array}{l}-\Delta H_{\alpha-\beta} \\
(\mathrm{kJ} / \mathrm{mol})\end{array}$ & $\begin{array}{r}-\Delta H_{\beta-\alpha} \\
(\mathrm{kJ} / \mathrm{mol})\end{array}$ & $\begin{array}{c}-\Delta S_{\alpha-\beta} \\
(J / K / m o l)\end{array}$ & $\begin{array}{c}-\Delta S_{\beta-\alpha} \\
(J / K / m o l)\end{array}$ & $\begin{array}{c}\mathbf{T}_{\mathbf{C}} \\
(\mathbf{K})\end{array}$ & $\begin{array}{l}-\mathbf{E}_{\mathrm{A} \alpha-\beta} \\
(\mathrm{kJ} / \mathrm{mol})\end{array}$ & $\begin{array}{l}-\mathrm{E}_{\mathrm{A} \beta-\alpha} \\
(\mathrm{kJ} / \mathrm{mol})\end{array}$ & Ref \\
\hline $14 \mathrm{~nm}$ & $27.4 \pm 0.2$ & $33.4 \pm 0.3$ & $61.5 \pm 0.5$ & $75.8 \pm 0.8$ & 416.1 & $18.0 \pm 0.1$ & $47.1 \pm 0.2$ & \\
\hline $32 \mathrm{~nm}$ & $28.5 \pm 0.2$ & $35.5 \pm 0.4$ & $66.9 \pm 0.6$ & $82.3 \pm 0.8$ & 455.5 & $20.2 \pm 0.1$ & $50.6 \pm 0.3$ & \\
\hline $65 \mathrm{~nm}$ & $29.9 \pm 0.3$ & $37.6 \pm 0.4$ & $72.6 \pm 0.6$ & $88.2 \pm 0.9$ & 495.4 & $22.6 \pm 0.2$ & $54.4 \pm 0.3$ & \\
\hline $110 \mathrm{~nm}$ & $32.9 \pm 0.3$ & $39.6 \pm 0.3$ & $82.4 \pm 0.9$ & $94.4 \pm 0.9$ & 558.2 & $25.3 \pm 0.3$ & $60.5 \pm 0.4$ & \\
\hline $\begin{array}{c}65 \mathrm{~nm} \\
\text { Film }\end{array}$ & $35.4 \pm 0.3$ & $40.9 \pm 0.3$ & $89.8 \pm 0.8$ & $100.3 \pm 1.1$ & 532.0 & $25.1 \pm 0.1$ & $61.0 \pm 0.4$ & \\
\hline Bulk Pd & - & - & - & - & & 25.1 & 62.7 & 19 \\
\hline Bulk Pd & $36.4 \pm 0.8$ & $41.3 \pm 0.8$ & $90.8 \pm 1.6$ & $99.4 \pm 1.6$ & 564.0 & & & 20 \\
\hline
\end{tabular}

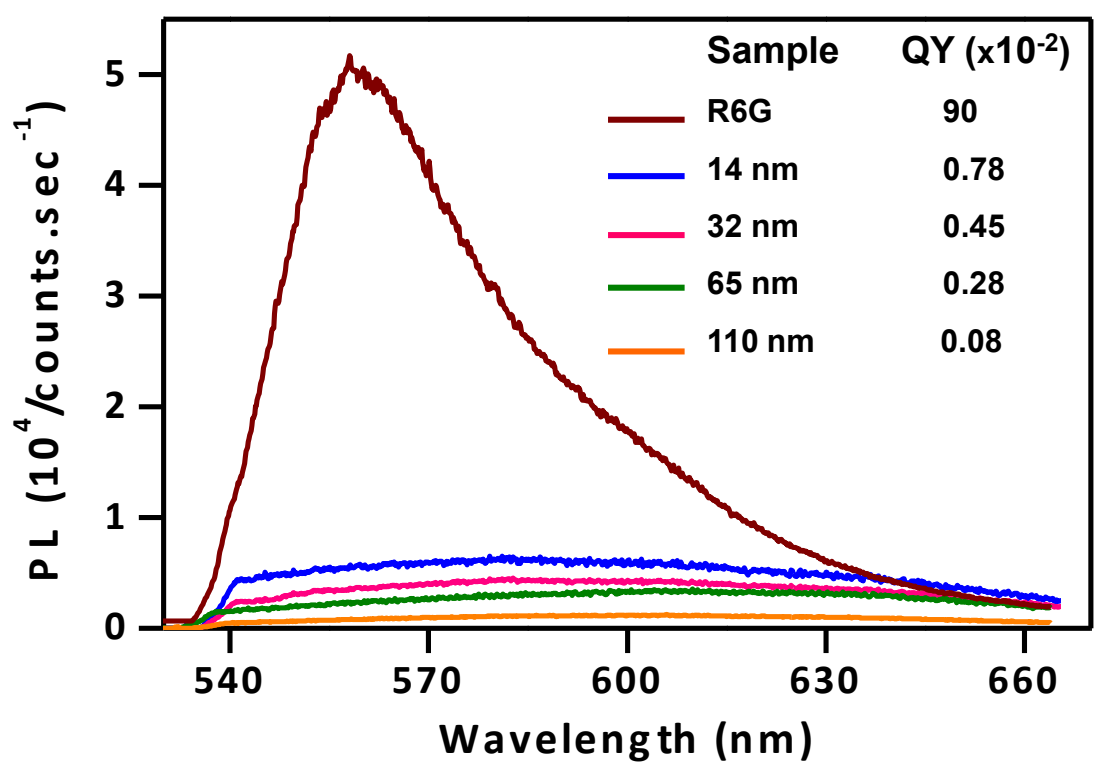

Figure S1. Photoluminescence (PL) emission of Pd nanocubes of different sizes compared to reference sample of Rhodamine 6G (R6G). Corresponding quantum yield (QY) values are shown in the inset. 


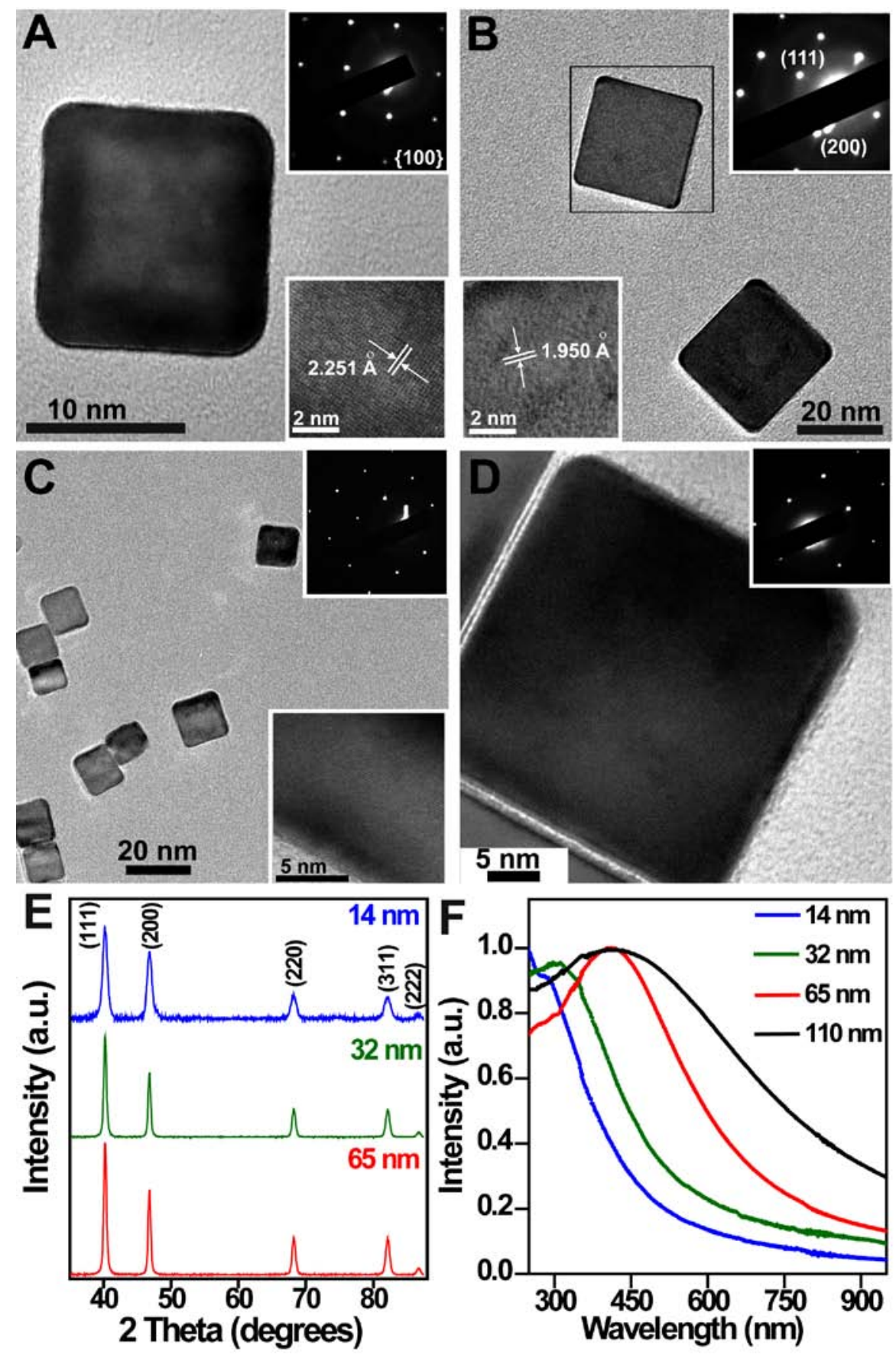

Figure S2. Low-magnification and-high magnification (inset) TEM images and corresponding electron diffraction images of nanocubes before hydriding for (A) $14 \mathrm{~nm}$ and (B) $32 \mathrm{~nm}$ nanocubes. Images after 3 cycles of hydriding and dehydriding are also shown for (C) $14 \mathrm{~nm}$ and (D) $32 \mathrm{~nm}$ nanocubes showing that they retain their shape and crystalline form after hydrogen cycling, and that they are single-crystalline entities bound by $\{100\}$ facets. In (B) the high magnification image and electron diffraction is from the cube outlined by the black square. (E) XRD patterns of Pd nanocubes of different sizes. XRD samples were prepared by drop-casting a dilute aqueous suspension of the nanocubes on silicon substrates. (F) Extinction spectra of $\mathrm{Pd}$ nanocubes dispersed in aqueous media showing a red-shift in the plasmon resonance from $280-423 \mathrm{~nm}$ with increasing nanocube size. 
The TEM, electron diffraction (ED), and XRD data clearly demonstrate that the nanocubes studied here are single-crystalline objects bound by $\{100\}$ facets. This strongly suggests that $\mathrm{H}_{2}$ absorption preferentially occurs along the planes of this crystal facet. TEM and ED images indicate nanocubes retain their morphology and remain single-crystalline after 3 cycles of hydriding and dehydriding. After hydriding, the XRD peaks of Pd shift to smaller $2 \theta$ values as expected for $\beta-\mathrm{PdH}_{0.6}$ (shown in Fig. S13).
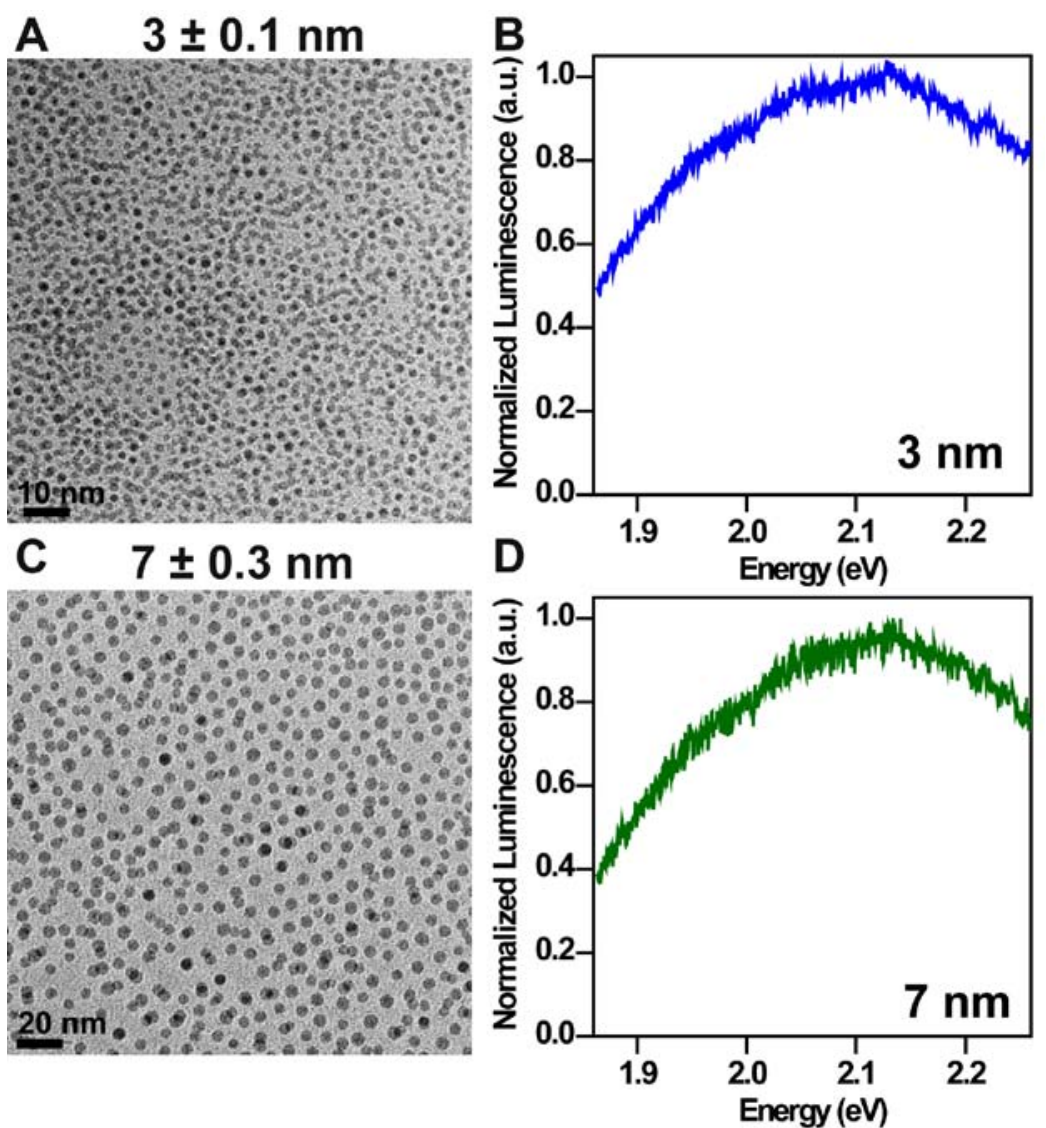

Figure S3: TEM micrographs and corresponding luminescence spectrum of $3 \pm 0.1 \mathrm{~nm}$ and $7 \pm 0.3 \mathrm{~nm} \mathrm{Pd}$ nanocrystals. 

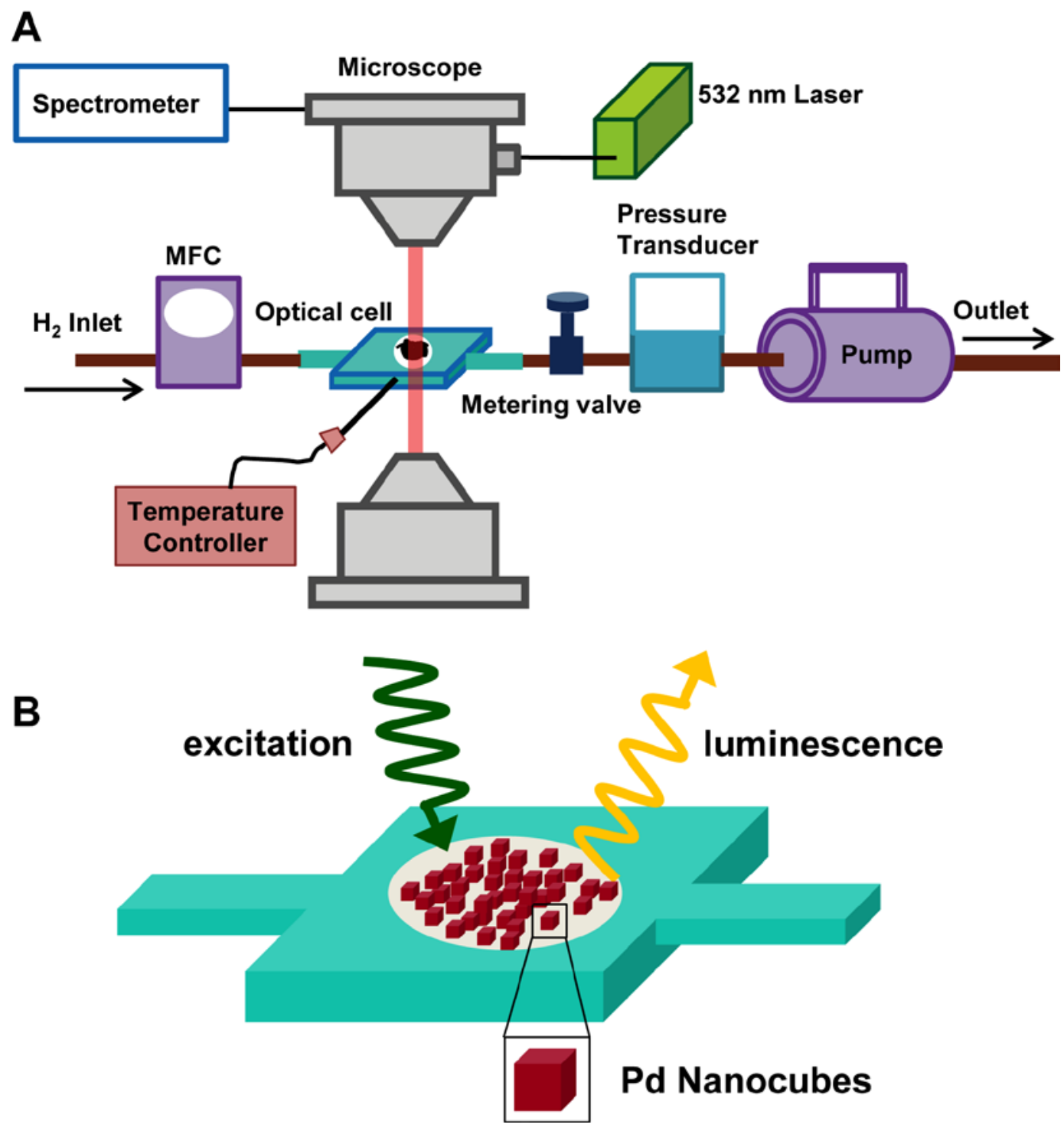

Figure S4. (A) Schematic representation of the in situ optical setup for hydriding and dehydriding. $\mathrm{H}_{2}$ gas is connected to a mass flow controller (MFC) to control the gas flow and then connected to a gas-tight optical cell with transparent windows. Pd nanocube/film samples are loaded in the optical cell and mounted on a confocal microscope. The microscope is attached to a $532 \mathrm{~nm}$ diode laser for excitation and spectrometer for detecting luminescence signals. Cartridge heaters are connected to the optical cell to controllably heat the samples and connected to a temperature controller for accurate readout. The thermocouple attached to the temperature controller is kept in contact with the substrates where Pd samples are loaded to ensure precise temperature readings are recorded. The optical cell is then connected to a pressure transducer with a digital readout and a low-flow metering valve is used to manually control the pressure of the cell during hydriding and dehydriding. The system is connected to a scroll pump and connected to the exhaust. (B) A detailed view of the optical cell with Pd nanocube samples loaded on the transparent quartz windows for optical excitation and subsequent emission collection. 

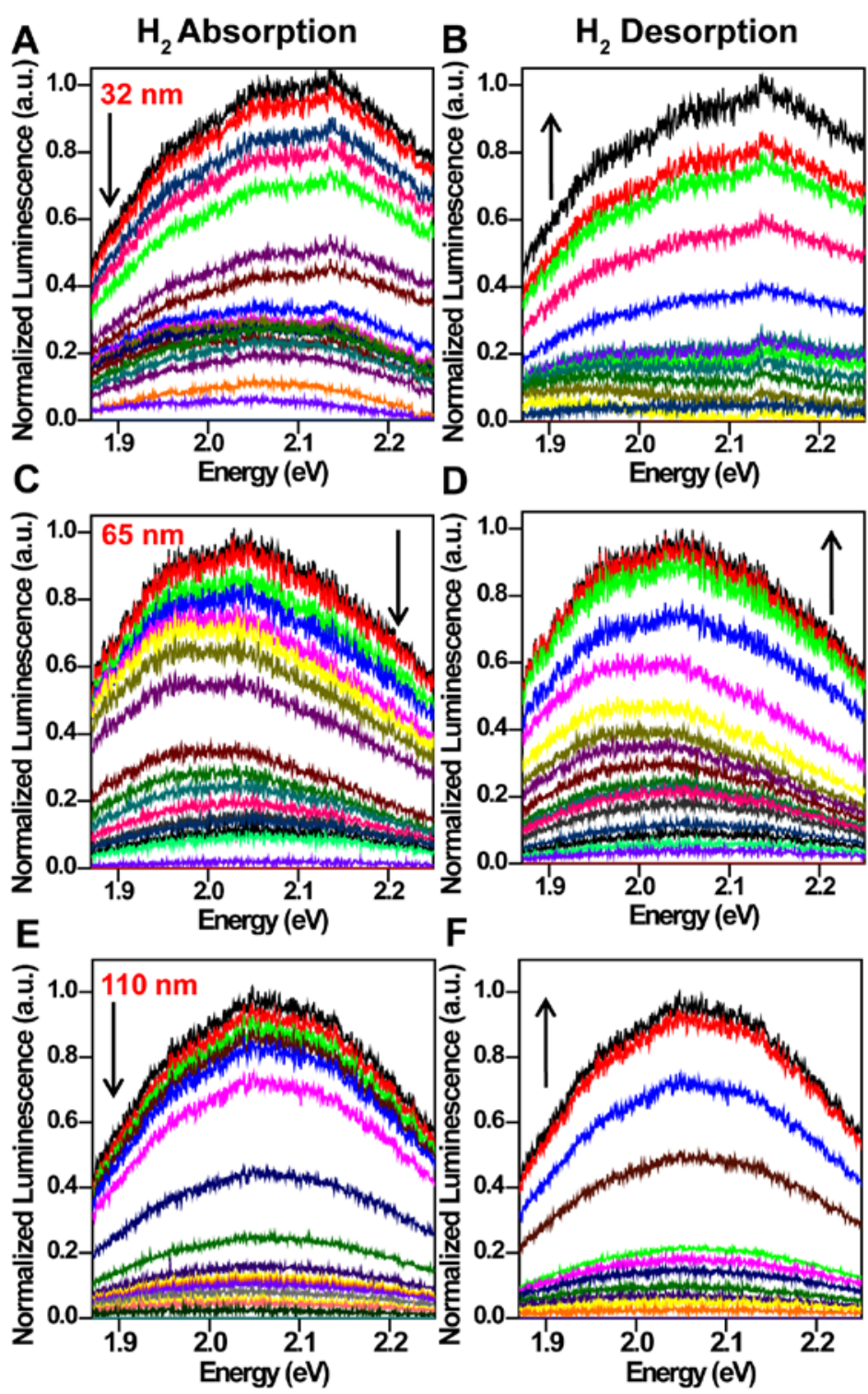

Figure S5. Normalized luminescence spectra recorded at $22{ }^{\circ} \mathrm{C}$ demonstrating decreased luminescence during $\mathrm{H}_{2}$ absorption and increased luminescence during $\mathrm{H}_{2}$ desorption of (A-B) $32 \mathrm{~nm}$, (C-D) $65 \mathrm{~nm}$, and (E-F) $110 \mathrm{~nm}$ Pd nanocubes, respectively. The size of the nanocubes is indicated on the upper-left hand corner in red text. The luminescence is normalized by dividing the luminescence intensity of the Pd metallic-phase by the fully hydrided phase.

The photoluminescence spectra were slightly shifted to lower energies with increasing size of the nanocubes, which may be attributed to the red-shift in the plasmon resonances (Figure S2) of the nanocubes with increasing size. Since the observed luminescence originates from the radiative decay of 
surface plasmons, a shift in the plasmon resonance with increasing size is expected to shift the emission maximum to lower energies (higher wavelengths).

To compute the pressure-luminescence isotherms (Figure 2 and Figure S7) luminescence signal was measured at $2.1 \mathrm{eV}(590 \mathrm{~nm})$ for the $14 \mathrm{~nm}$ and $32 \mathrm{~nm}$ nanocubes, and at $2.07 \mathrm{eV}(600 \mathrm{~nm})$ for the 65 $\mathrm{nm}$ and $110 \mathrm{~nm}$ nanocubes.
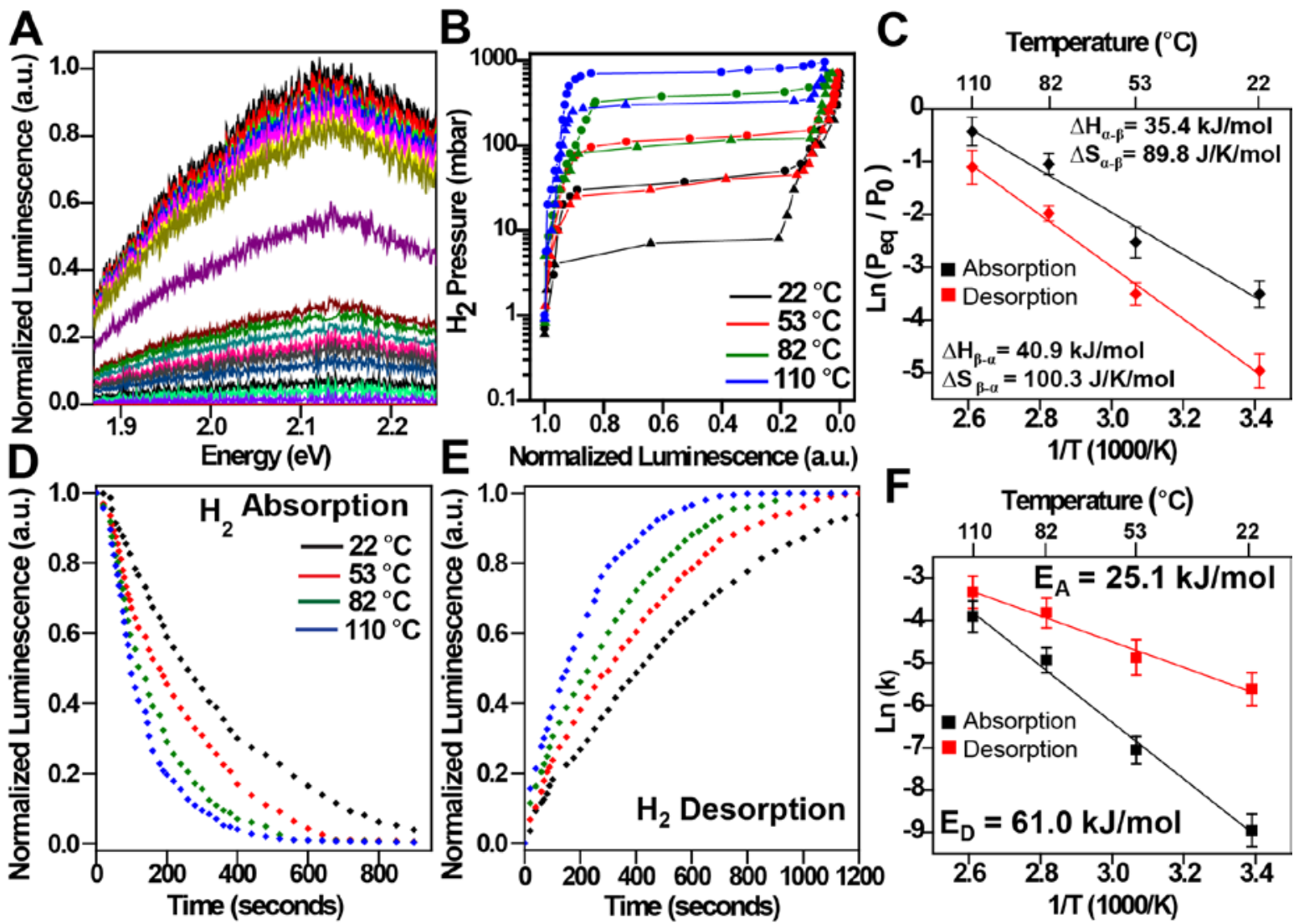

Figure S6. Bulk-like behavior observed in hydriding/dehydriding thermodynamics and kinetics study of $65 \mathrm{~nm}$ Pd film. (A) Normalized luminescence spectra at $22{ }^{\circ} \mathrm{C}$ decreasing during $\mathrm{H}_{2}$ absorption and increasing during $\mathrm{H}_{2}$ desorption. (B) Pressure-Luminescence (P-L) isotherms at different temperatures obtained by measuring changes in luminescence with increasing pressure during hydriding (circles) and decreasing pressure during dehydriding (triangles). The isotherms are normalized by mass of the films deposited to measure the luminescence changes. (C) Van't Hoff plots showing linearity between the logarithm of the equilibrium plateau pressures and inverse absolute temperature. The error bars are calculated based upon three independent absorption and desorption measurements performed on each sample. $\Delta \mathrm{H}$ and $\Delta \mathrm{S}$ are calculated from the slope and intercept of the linear fit, respectively. $\mathrm{H}_{2}$ absorption (D) and desorption (E) kinetics showing a decrease in normalized luminescence intensity during absorption and increase during desorption as a function of time. (F) Arrhenius rate law plots, the logarithm of the rate constant $(\ln k)$ as a function of inverse temperature $(1 / T)$, showing linear relationship 
for the Pd film. The error bars are calculated based on three independent absorption and desorption measurements. The activation energy $\left(\mathrm{E}_{\mathrm{A}}\right)$ is calculated from the slope of this linear fit.
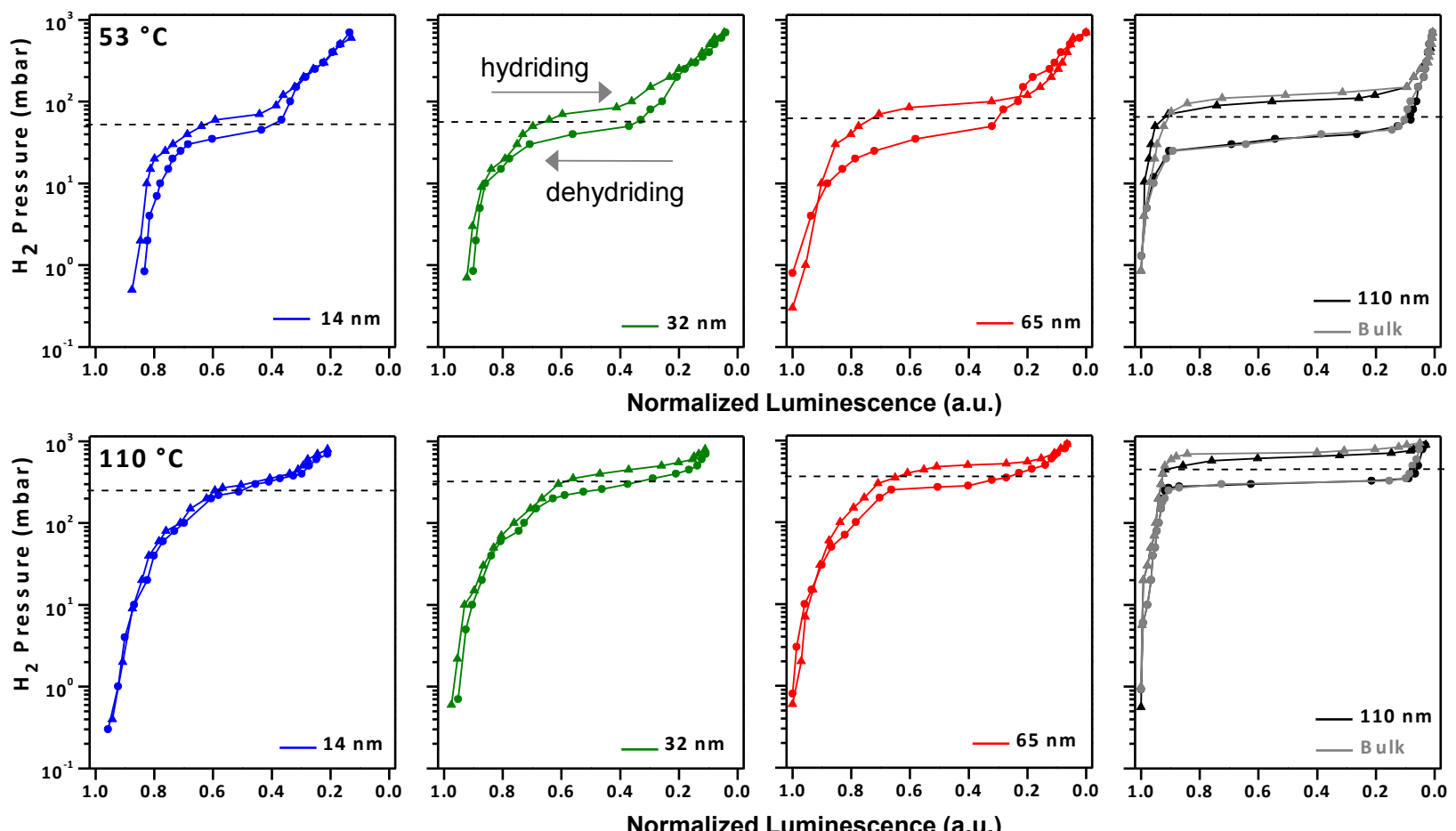

Figure S7. Pressure-Luminescence isotherms during hydriding and dehydriding of Pd nanocubes at $53{ }^{\circ} \mathrm{C}$ (top) and $110{ }^{\circ} \mathrm{C}$ (bottom) are shown. The size of the nanocubes is indicated in each plot. The isotherms are normalized by mass of the nanocubes deposited to measure the luminescence changes. The mid-point, $\mathrm{P}_{\text {mid, }}$ of each isotherm is shown with a dotted line. The P-L isotherm of bulk film is also shown for comparison.

The $\mathrm{P}_{\text {mid }}$ for each isotherm were calculated by two different methods. Figure S8-A shows the P-L isotherm of $32 \mathrm{~nm} \mathrm{Pd}$ nanocubes and visual representation of how mid-points were evaluated. In the first scenario, hydriding $\left(\mathrm{P}_{\text {hyd }}\right)$ and dehydriding $\left(\mathrm{P}_{\text {dehyd }}\right)$ pressures were evaluated at luminescence 0.5 , shown by a green drop-line in Figure $\mathrm{S} 8 . \mathrm{P}_{\text {hyd }}$, and $\mathrm{P}_{\text {dehyd }}$ were measured at the intersection of the green drop-line and the forward and backward isotherms respectively. $\operatorname{Ln}\left(\mathrm{P}_{\mathrm{mid}}\right)$ was calculated by taking an average of $\operatorname{Ln}\left(\mathrm{P}_{\text {hyd }}\right)$, and $\operatorname{Ln}\left(\mathrm{P}_{\text {dehyd }}\right): \operatorname{Ln}\left(\mathrm{P}_{\text {mid }}\right)=\left(\operatorname{Ln}\left(\mathrm{P}_{\text {hyd }}\right)+\operatorname{Ln}\left(\mathrm{P}_{\text {dehyd }}\right)\right) / 2 . \quad \mathrm{P}_{\text {mid }}$ was then calculated as $\mathrm{P}_{\text {mid }}=\mathrm{e}^{\operatorname{Ln}(\mathrm{Pmid})}$.

In the second scenario, $\mathrm{P}_{\text {mid }}$ was evaluated by taking the mid-point of the two extremes of the isotherm where hydriding and dehydriding start, shown by gray drop-lines. Depending on the size of the nanocube and the temperature of the experiment, this mid-point was often at luminescence 0.5 or slightly 
higher. In the case of $32 \mathrm{~nm} \mathrm{Pd}$ nanocubes at $82{ }^{\circ} \mathrm{C}$, this mid-point was at luminescence 0.528 . $\mathrm{P}_{\text {hyd }}$ and $\mathrm{P}_{\text {dehyd }}$ were measured once again at the intersection of the gray drop-line and the forward and backward isotherms respectively. $\operatorname{Ln}\left(\mathrm{P}_{\text {mid }}\right)$ was calculated by taking an average of $\operatorname{Ln}\left(\mathrm{P}_{\text {hyd }}\right)$, and $\operatorname{Ln}\left(\mathrm{P}_{\text {dehyd }}\right)$ : $\operatorname{Ln}\left(P_{\text {mid }}\right)=\left(\operatorname{Ln}\left(P_{\text {hyd }}\right)+\operatorname{Ln}\left(P_{\text {dehyd }}\right)\right) / 2$. $P_{\text {mid }}$ was then calculated as $P_{\text {mid }}=e^{\operatorname{Ln}(P m i d)}$. Modified Van't Hoff plots were then plotted by normalizing the $\operatorname{Ln}\left(\mathrm{P}_{\text {mid }}\right)$ of nanocubes with bulk Pd i.e. $\Delta G_{\text {norm }}=\operatorname{Ln}\left(\mathrm{P}_{\text {mid-nano }}\right)-$ $\operatorname{Ln}\left(\mathrm{P}_{\text {mid-Bulk }}\right)$ for all nanocube sizes at all temperatures. By calculating the $\mathrm{P}_{\text {mid }}$ by two different methods we clearly show that the trends in Fig. S8 (B) and (C) are qualitatively very similar, indicating that making nanocubes smaller acts to thermodynamically favor the metal hydride phase over the metal one, the more so as temperature increases. 

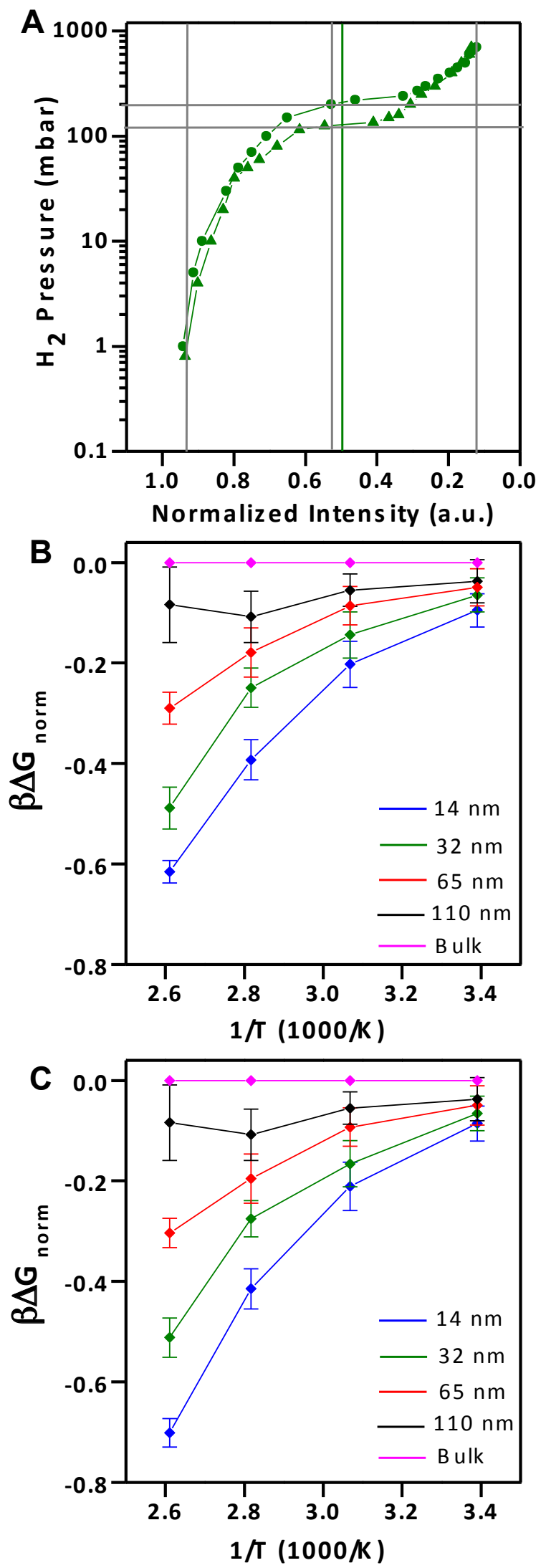
Figure S8. (A) P-L isotherm of $32 \mathrm{~nm} \mathrm{Pd}$ nanocube at $82{ }^{\circ} \mathrm{C}$ with drop lines in gray to show the two extremes of the isotherm where hydriding and dehydriding starts and the mid-point of these at luminescence 0.528 for this system. A drop line is also indicated in green at luminescence 0.5 . The hydriding and dehydriding pressures (shown by horizontal gray lines) were evaluated separately at the two different luminescence points respectively. $\operatorname{Ln}\left(\mathrm{P}_{\text {mid }}\right)$ were calculated by taking an average of the logarithmic of the hydriding and dehydriding pressures $\operatorname{Ln}\left(\mathrm{P}_{\text {mid }}\right)=\left(\operatorname{Ln}\left(\mathrm{P}_{\text {hyd }}\right)+\operatorname{Ln}\left(\mathrm{P}_{\text {dehyd }}\right)\right) / 2$ for all nanocube sizes and bulk film. The mid-point pressure was then calculated as $\mathrm{P}_{\text {mid }}=\mathrm{e}^{\mathrm{Ln}(\mathrm{Pmid})}$ Modified Van't hoff plots were then derived at luminescence 0.5 (B) and the other luminescence (C) normalized by bulk film behavior (the vertical axis is bulk-normalized free energy change, $\Delta G_{\text {norm }}=\ln \left(P_{\text {mid }} / P_{\text {mid }}^{\text {film }}\right)$. Error bars are calculated from 5 independent measurements. By calculating the $\operatorname{Ln}\left(\mathrm{P}_{\text {mid }}\right)$ by two different methods we clearly show that the trends in (B) and (C) are qualitatively very similar, indicating that making nanocubes smaller acts to thermodynamically favor the metal hydride phase over the metal one, the more so as temperature increases. 
Table S2: $\mathrm{P}_{\text {hyd }}, \mathrm{P}_{\text {dehyd }}, \mathrm{P}_{\text {mid }}$, and their corresponding logarithmic values are represented here for nanocubes of all sizes and bulk $\mathrm{Pd}$ film evaluated at luminescence at 0.5 . This data is averaged over five measurements for all nanocube sizes at all temperatures. $\beta \Delta \mathrm{G}_{\text {norm }}=\left(\operatorname{Ln}\left(\mathrm{P}_{\text {mid }}{ }^{-n a n o}\right)-\operatorname{Ln}\left(\mathrm{P}_{\text {mid-bulk }}\right)\right)$ used to plot the modified Van't Hoff plots (Fig. 2b and Fig. S8) are also shown.

\begin{tabular}{|c|c|c|c|c|c|c|c|c|}
\hline \multicolumn{9}{|c|}{$14 \mathrm{~nm}$} \\
\hline Temp & $1 / T \times 10^{3}$ & $\mathbf{P}_{\text {hyd }}$ & $\operatorname{Ln}\left(\mathbf{P}_{\text {hyd }}\right)$ & $\mathbf{P}_{\text {dehyd }}$ & $\operatorname{Ln}\left(\mathbf{P}_{\text {dehyd }}\right)$ & $\operatorname{Ln}\left(\mathbf{P}_{\text {mid }}\right)$ & $\mathbf{P}_{\text {mid }}$ & $\begin{array}{l}\operatorname{Ln}\left(\mathbf{P}_{\text {mid"nano }}\right)- \\
\operatorname{Ln}\left(\mathbf{P}_{\text {mid-Bulk }}\right)\end{array}$ \\
\hline 22 & 3.3898305 & 0.0215 & -3.83970 & 0.0105 & -4.556380 & -4.1980411 & 0.01502498 & -0.095021801 \\
\hline 53 & 3.0674846 & 0.0684 & -2.68238 & 0.0447 & -3.107782 & -2.8950821 & 0.05529448 & -0.202315916 \\
\hline 82 & 2.81690140 & 0.165 & -1.80181 & 0.122 & -2.10373 & -1.952772 & 0.14188023 & -0.392804081 \\
\hline 110 & 2.61096605 & 0.294 & -1.22417 & 0.241 & -1.422958 & -1.3235669 & 0.26618415 & -0.615355066 \\
\hline \multicolumn{9}{|c|}{$32 \mathrm{~nm}$} \\
\hline Temp & $1 / T \times 10^{3}$ & $\mathbf{P}_{\text {hyd }}$ & $\operatorname{Ln}\left(\mathbf{P}_{\mathrm{hyd}}\right)$ & $\mathbf{P}_{\text {dehyd }}$ & $\operatorname{Ln}\left(\mathbf{P}_{\text {dehyd }}\right)$ & $\operatorname{Ln}\left(\mathbf{P}_{\text {mid }}\right)$ & $\mathbf{P}_{\text {mid }}$ & 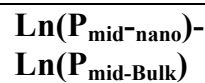 \\
\hline 22 & 3.3898305 & 0.0258 & -3.65738 & 0.0093 & -4.677740 & -4.1675608 & 0.01549 & -0.064541452 \\
\hline 53 & 3.0674846 & 0.0812 & -2.5108 & 0.0423 & -3.162968 & -2.8369041 & 0.05860683 & -0.144137912 \\
\hline 82 & 2.81690140 & 0.208 & -1.57021 & 0.129 & -2.047942 & -1.8090800 & 0.16380476 & -0.249112098 \\
\hline 110 & 2.61096605 & 0.358 & -1.02722 & 0.255 & -1.366491 & -1.1968570 & 0.30214235 & -0.48864515 \\
\hline \multicolumn{9}{|c|}{$65 \mathrm{~nm}$} \\
\hline Temp & $1 / T \times 10^{3}$ & $\mathbf{P}_{\text {hyd }}$ & $\operatorname{Ln}\left(\mathbf{P}_{\text {hyd }}\right)$ & $\mathbf{P}_{\text {dehyd }}$ & $\operatorname{Ln}\left(\mathbf{P}_{\text {dehyd }}\right)$ & $\mathbf{L n}\left(\mathbf{P}_{\text {mid }}\right)$ & $\mathbf{P}_{\text {mid }}$ & $\begin{array}{l}\operatorname{Ln}\left(\mathbf{P}_{\text {mid"nano }}\right)- \\
\operatorname{Ln}\left(\mathbf{P}_{\text {mid-Bulk }}\right)\end{array}$ \\
\hline 22 & 3.3898305 & 0.0313 & -3.46413 & 0.0079 & -4.840892 & -4.1525148 & 0.01572482 & -0.049495469 \\
\hline 53 & 3.0674846 & 0.0923 & -2.38271 & 0.0418 & -3.174858 & -2.7787850 & 0.06211393 & -0.086018838 \\
\hline 82 & 2.81690140 & 0.255 & -1.36649 & 0.121 & -2.111964 & -1.7392282 & 0.17565591 & -0.179260295 \\
\hline 110 & 2.61096605 & 0.505 & -0.68319 & 0.269 & -1.313043 & -0.9981203 & 0.36857157 & -0.289908511 \\
\hline \multicolumn{9}{|c|}{$110 \mathrm{~nm}$} \\
\hline Temp & $1 / T \times 10^{3}$ & $\mathbf{P}_{\text {hyd }}$ & $\operatorname{Ln}\left(\mathbf{P}_{\mathrm{hyd}}\right)$ & $\mathbf{P}_{\text {dehyd }}$ & $\operatorname{Ln}\left(\mathbf{P}_{\text {dehyd }}\right)$ & $\operatorname{Ln}\left(\mathbf{P}_{\text {mid }}\right)$ & $\mathbf{P}_{\text {mid }}$ & 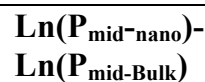 \\
\hline 22 & 3.3898305 & 0.0352 & -3.34670 & 0.0072 & -4.933674 & -4.1401917 & 0.0159198 & -0.037172343 \\
\hline 53 & 3.0674846 & 0.1134 & -2.17683 & 0.0362 & -3.318696 & -2.7477650 & 0.0640709 & -0.054998824 \\
\hline 82 & 2.81690140 & 0.294 & -1.22417 & 0.121 & -2.111964 & -1.6680701 & 0.18861071 & -0.108102184 \\
\hline 110 & 2.61096605 & 0.668 & -0.40346 & 0.307 & -1.180907 & -0.7921873 & 0.45285318 & -0.083975455 \\
\hline \multicolumn{9}{|l|}{ Bulk } \\
\hline Temp & $1 / T \times 10^{3}$ & $\mathbf{P}_{\text {hyd }}$ & $\operatorname{Ln}\left(P_{\text {hyd }}\right)$ & $\mathbf{P}_{\text {dehyd }}$ & $\operatorname{Ln}\left(\mathbf{P}_{\text {dehyd }}\right)$ & $\operatorname{Ln}\left(\mathbf{P}_{\text {mid }}\right)$ & $\mathbf{P}_{\text {mid }}$ & $\begin{array}{l}\operatorname{Ln}\left(\mathbf{P}_{\text {mid }}{ }^{-} \text {nano }\right)- \\
\operatorname{Ln}\left(\mathbf{P}_{\text {mid-Bulk }}\right)\end{array}$ \\
\hline 22 & 3.3898305 & 0.0364 & -3.31318 & 0.0075 & -4.892852 & -4.1030193 & 0.01652271 & 0 \\
\hline 53 & 3.0674846 & 0.128 & -2.05572 & 0.0358 & -3.329807 & -2.6927662 & 0.06769343 & 0 \\
\hline 82 & 2.81690140 & 0.384 & -0.95711 & 0.115 & -2.162823 & -1.5599679 & 0.21014281 & 0 \\
\hline 110 & 2.61096605 & 0.78 & -0.24846 & 0.311 & -1.167962 & -0.7082118 & 0.49252411 & 0 \\
\hline
\end{tabular}



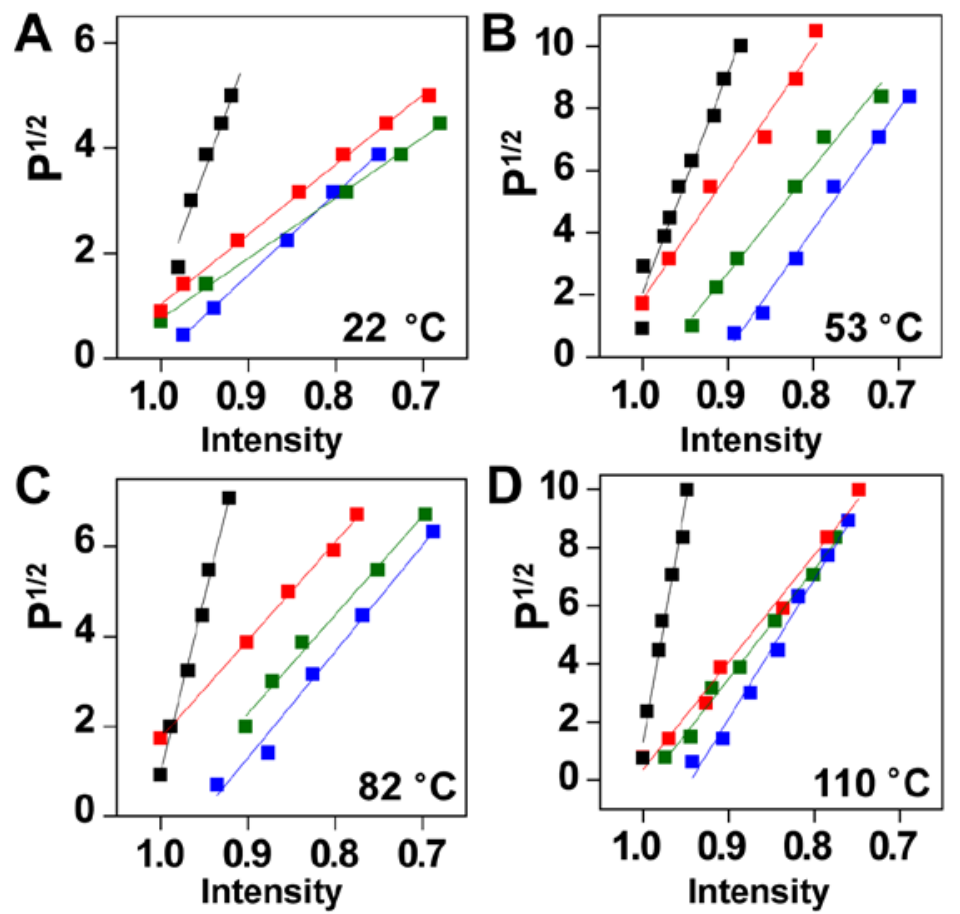

Figure S9. Sievert's Law plots showing linear scaling between square root of $\mathrm{H}_{2}$ pressure $\left(\mathrm{P}^{1 / 2}\right)$ and luminescence represented for all nanocube sizes at different temperatures.

Size-dependent trends in Van't Hoff plots are shown in Figure S10, suggesting that decrease in size results in lower thermodynamic barriers due to an increase in surface area, defects, interfaces, and grain boundaries which reduces the thermodynamic stability of the hydride-phase relative to metallicphase. 

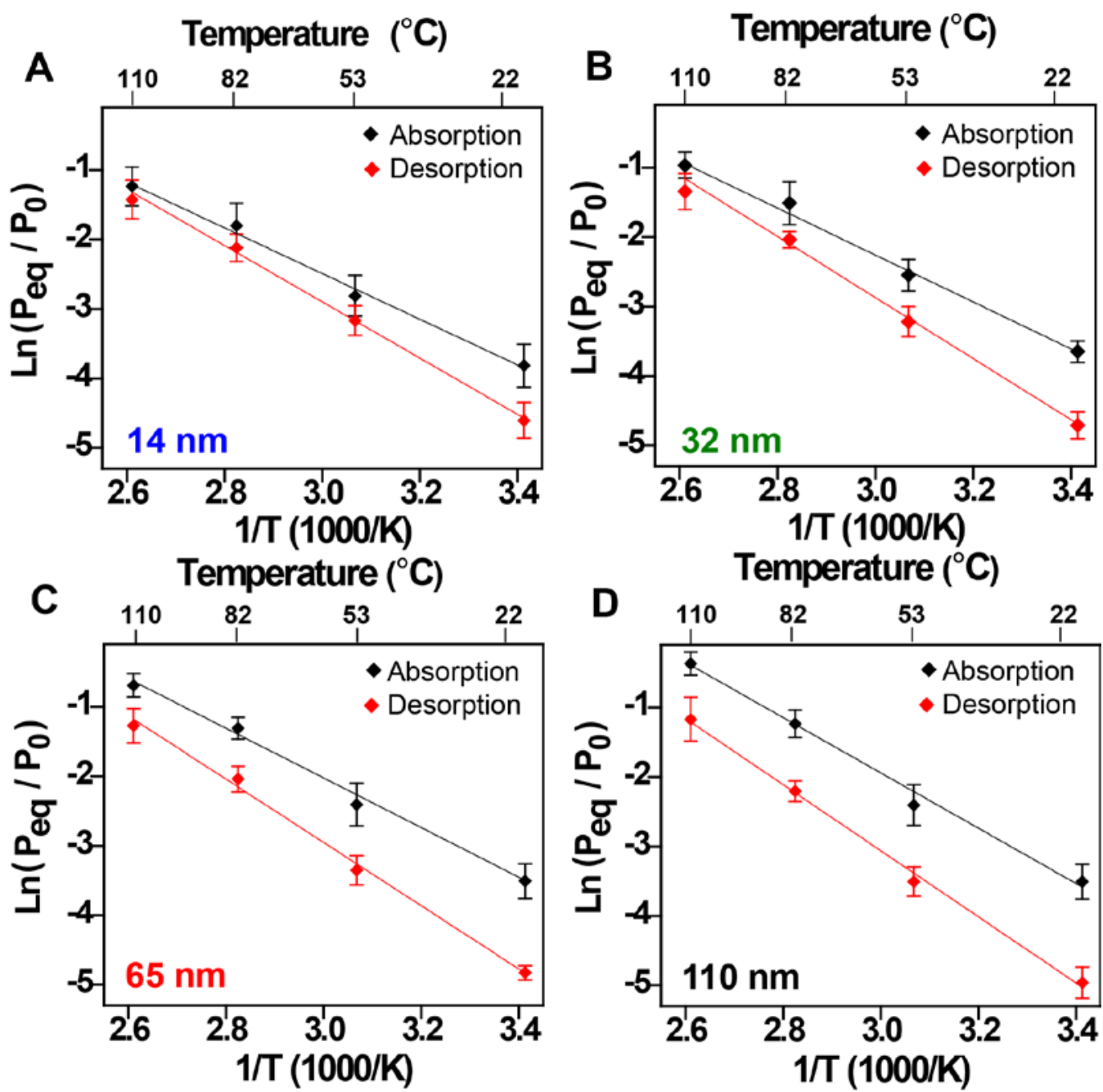

Figure S10. (A-D) Van't Hoff plots, obtained by taking the mid-point of plateau pressure of hydriding and dehydriding reaction respectively, are shown for all nanocube sizes. The size is indicated in each plot. The error bars are calculated based on three independent measurements. Enthalpy and entropy of hydrogen absorption and desorption are calculated from the slope and intercept of the plots respectively, shown in Table $\mathrm{S} 1$. Critical temperatures, $\mathrm{T}_{\mathrm{C}}$, were obtained from enthalpy and entropy of absorption and desorption given by, $T_{C}=\frac{\Delta H_{\alpha \rightarrow \beta}-\Delta H_{\beta \rightarrow \alpha}}{\Delta S_{\alpha \rightarrow \beta}-\Delta S_{\beta \rightarrow \alpha}}$.

Size-dependent $\mathrm{H}_{2}$ absorption and desorption kinetics at different temperatures are represented in Figure 5 and S11. To summarize: the smaller the nanocubes, the faster the hydriding and dehydriding behavior. The $14 \mathrm{~nm}$ nanocubes absorb $\mathrm{H}_{2}$ in 40 seconds at $110{ }^{\circ} \mathrm{C}$ and 300 seconds at $22{ }^{\circ} \mathrm{C}$, and desorb in 200 seconds and 500 seconds at $110^{\circ} \mathrm{C}$ and $22^{\circ} \mathrm{C}$ respectively. The $110 \mathrm{~nm}$ nanocrystals demonstrate slower kinetics compared to the smaller nanocubes controlled by the smaller surface to volume ratios and 
slower surface diffusion. The $110 \mathrm{~nm}$ nanocrystals hydride in 300 seconds at $110{ }^{\circ} \mathrm{C}$ and 900 seconds at $22{ }^{\circ} \mathrm{C}$, and dehydride in 600 seconds and 1300 seconds at $110^{\circ} \mathrm{C}$ and $22{ }^{\circ} \mathrm{C}$, respectively. The rate of hydriding and dehydriding observed in our experiments is reasonable since $\mathrm{H}_{2}$ absorption and desorption in bulk Pd films is on the order of minutes ${ }^{21}$ or hours. ${ }^{22}$
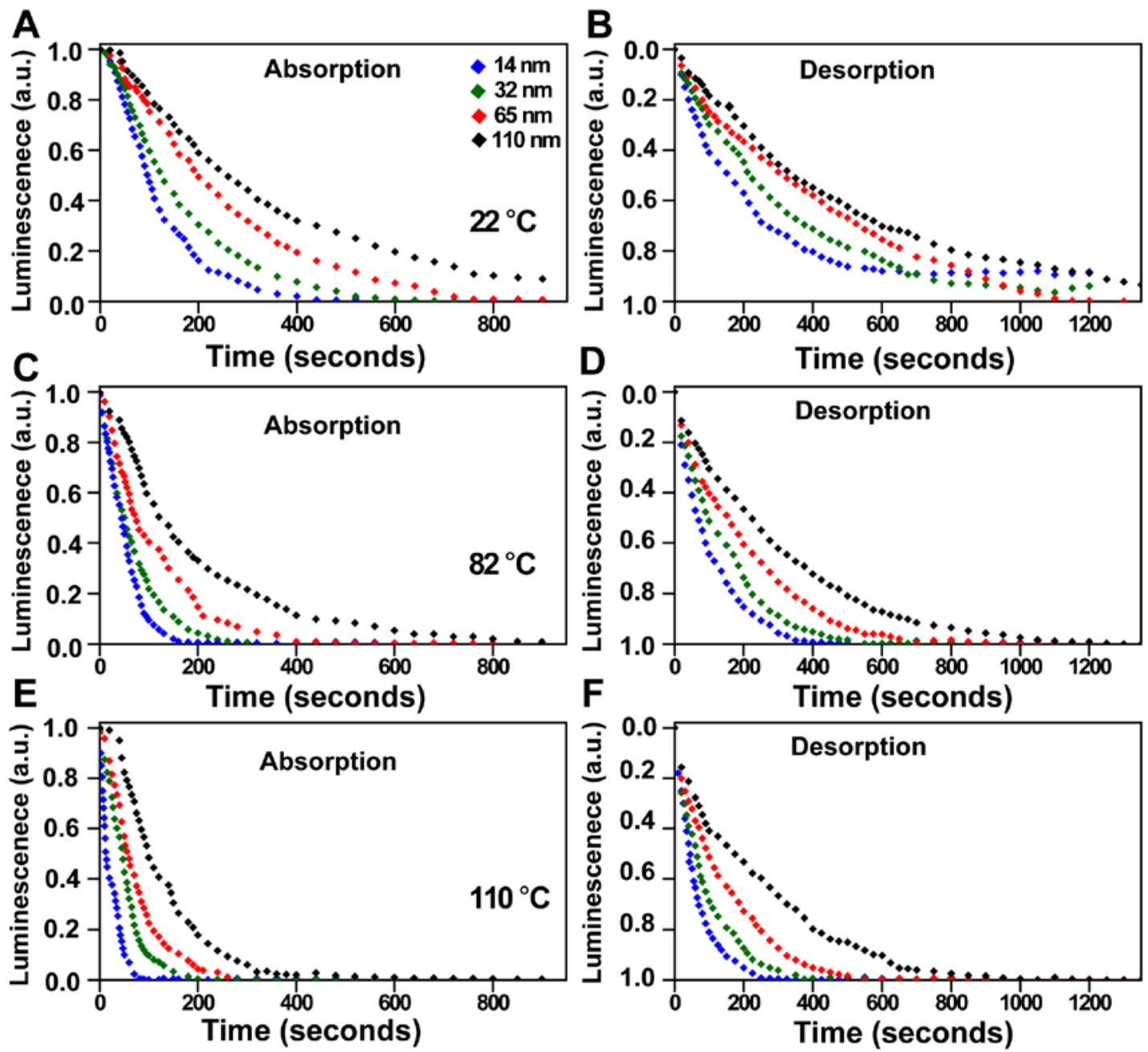

Figure S11. $\mathrm{H}_{2}$ absorption (left) and desorption (right) kinetics of Pd nanocubes of different sizes at (AB) $22{ }^{\circ} \mathrm{C}(\mathrm{C}-\mathrm{D}) 82{ }^{\circ} \mathrm{C}$ and $(\mathrm{E}-\mathrm{F}) 110^{\circ} \mathrm{C}$ showing a decrease in normalized luminescence intensity during absorption and reverse during desorption as a function of time. Note: the y-axis are reversed in the case of desorption. The size of the nanocubes in all plots is as indicated in (A). 

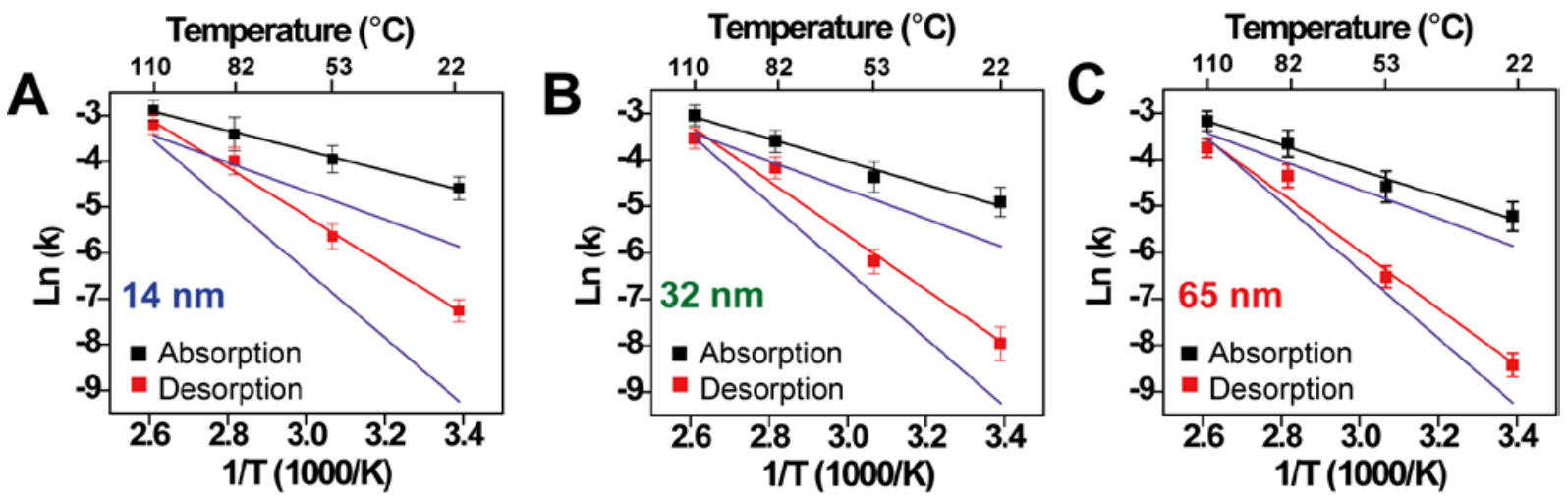

Figure S12. (A-C) Arrhenius rate law plots correlating rate constant and inverse temperature obtained from the exponential fits of the kinetic data. The nanocube size is indicated in each plot. The error bars are calculated based on three independent measurements. Rate law plots of $110 \mathrm{~nm} \mathrm{Pd} \mathrm{nanocrystals}$ (purple lines) are provided in each plot for comparison. The activation energies obtained from these plots are represented in Table $\mathrm{S} 1$.

In addition to changes in optical spectra, XRD patterns of the nanocubes were also observed to shift after hydriding from face-centered cubic (fcc) lattice of Pd to lower angles corresponding to cubic $\mathrm{PdH}_{0.6}$, indicating complete conversion to hydride phase (Fig. S13). Control experiments demonstrated that the observed Pd luminescence was not an artifact from the quartz background (Fig. S14) and that the measured changes in luminescence resulted from $\mathrm{H}_{2}$ sorption of $\mathrm{Pd}$ rather than substrate effects, or any remaining surfactant or due to pressure effects in the optical cell (Fig. S15). 

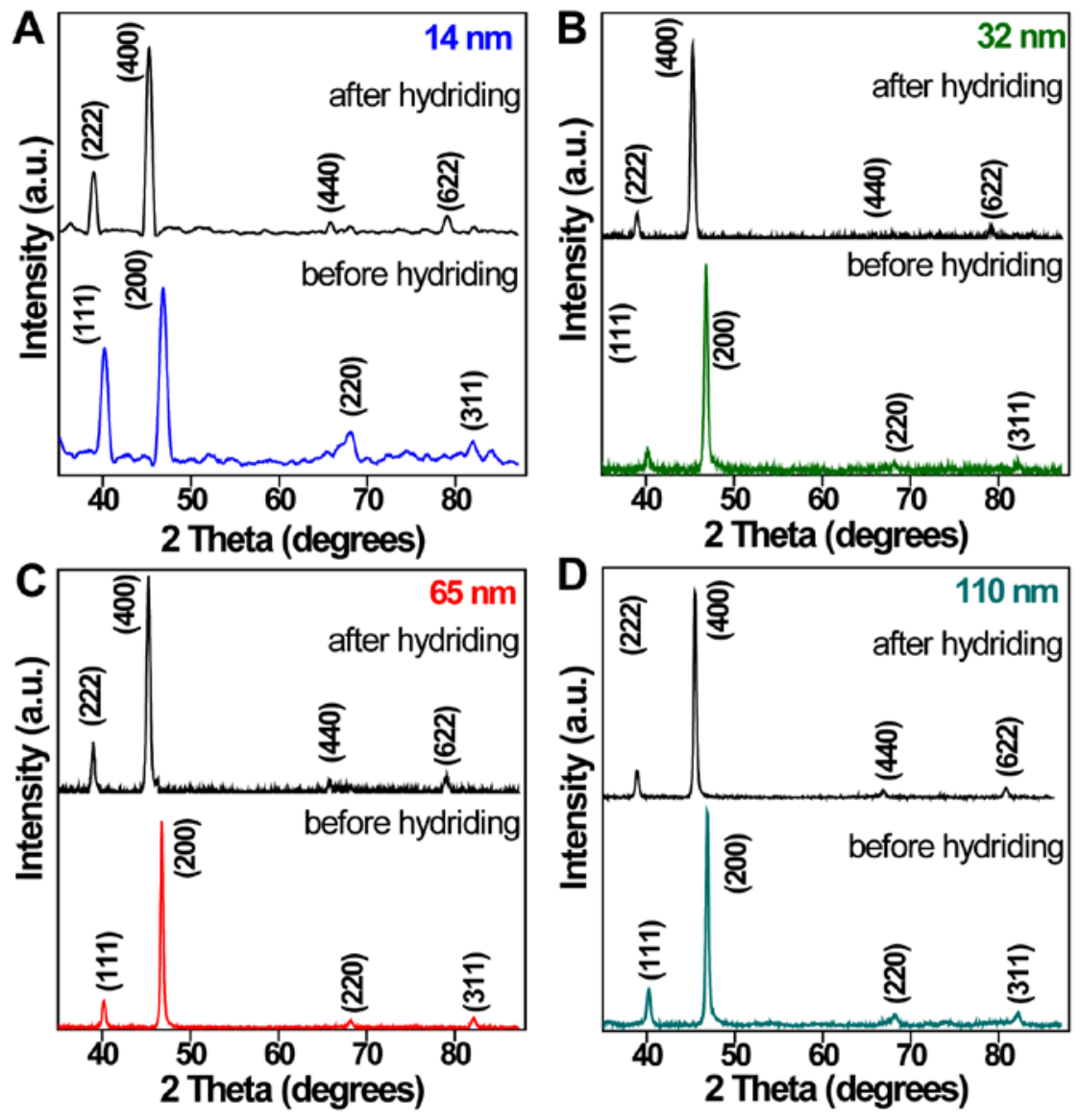

Figure S13. XRD patterns of nanocrystals before and after hydriding for 30 minutes. A clear shift in peaks from $\mathrm{Pd}$ (face-centered cubic, Fm-3m (225)) to $\beta-\mathrm{PdH}_{0.6}$ (cubic, $\mathrm{Pm}-3 \mathrm{n}$ (223)) is observable for all sizes. In each XRD pattern the intensity of the (200) peak of Pd is abnormally high relative to (111) peak, not observed usually in bulk Pd XRD pattern. This is attributed to the abundance of $\{100\}$ facets in the nanocubes; the $\{100\}$ planes had a propensity to preferentially align parallel to the surface of the supporting substrate forming short-range self-assembled arrays. This behavior was observable in SEM (Figure S14) and also has been reported previously for Ag nanocubes ${ }^{23}$. The self-assembled arrays are formed when a concentrated aqueous suspension is drop-casted on silicon or quartz substrate under vacuum. 

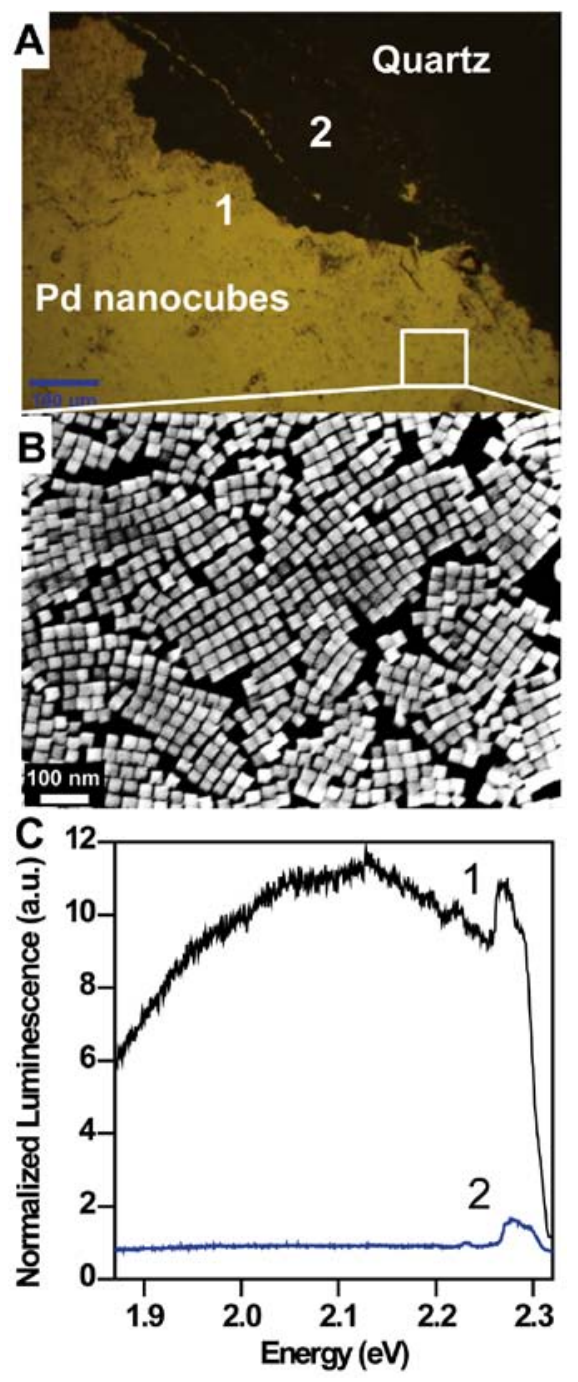

Figure S14. (A) Optical image of quartz substrate with $32 \mathrm{~nm}$ Pd nanocubes deposited and (B) SEM image of nanocubes within a selected area. Selected area is shown with a white square. (C) Luminescence of Pd nanocubes compared with quartz background. The luminescence is normalized to the intensity observed for quartz. The area of the substrate from where the spectra were obtained is indicated by numbers. The broad peak at $2.27 \mathrm{eV}$ corresponds to Si-O-Si stretching and bending modes from the quartz substrate. 

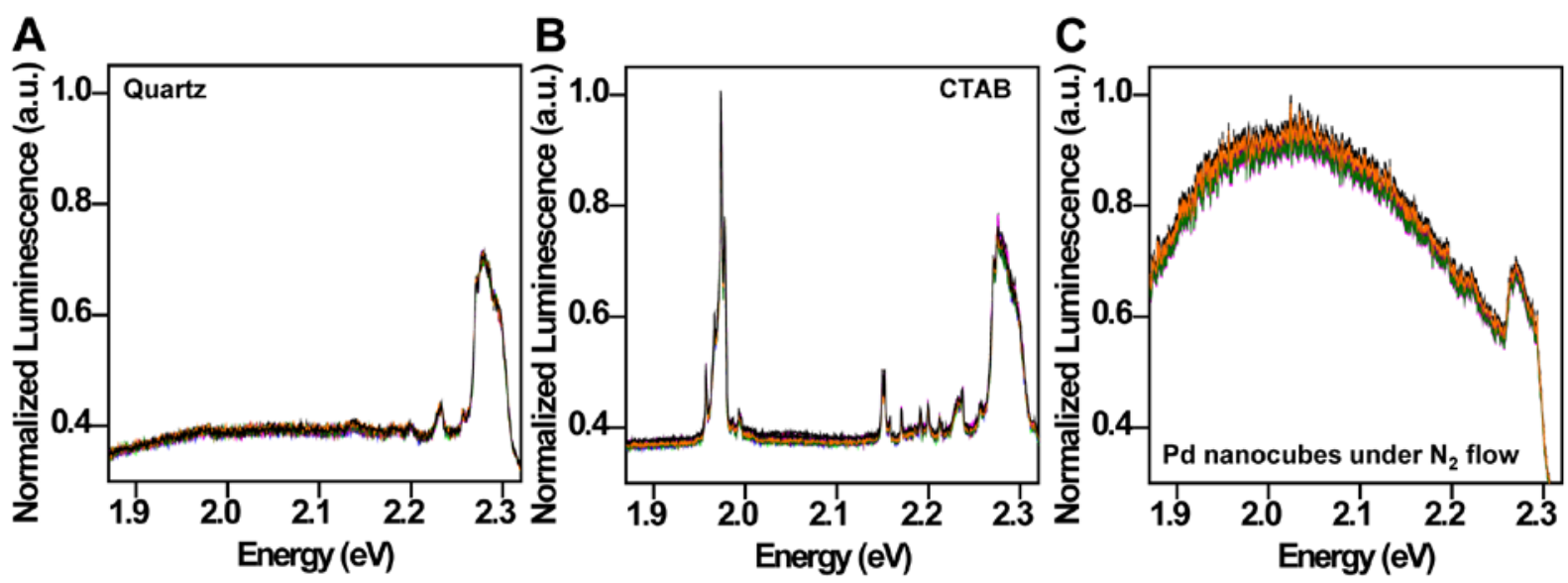

Figure S15. Control experiments at room temperature showing no change during (A) hydriding a plain quartz substrate, (B) hydriding CTAB deposited on quartz substrate and (C) $65 \mathrm{~nm}$ Pd nanocubes under nitrogen flow for 40 minutes. The broad peak at $2.27 \mathrm{eV}$ corresponds to Si-O-Si stretching and bending modes from the quartz substrate. All measurements were obtained with a $532 \mathrm{~nm}$ diode laser at equivalent excitation power $(5 \mathrm{~mW})$ and equivalent gas flow $(150 \mathrm{sccm} / \mathrm{min})$ for 40 minutes. 
A
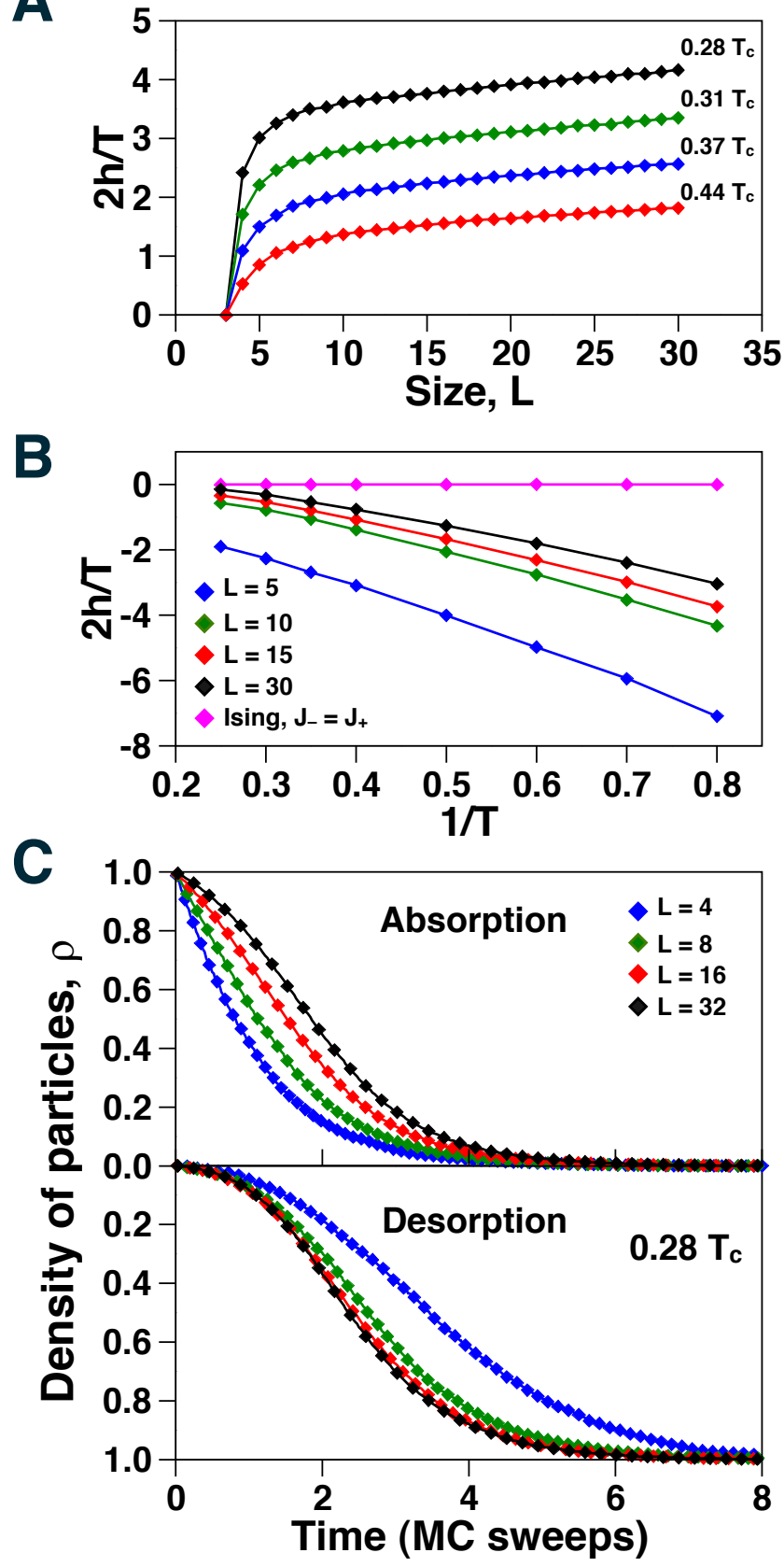

Figure S16. Artifacts associated with the lattice gas representation in the presence of an energetically inert wall (i.e. one that has no interaction with the metal- and metal hydride phases). Such a representation promotes an effective attraction between vacancies and the wall. Hysteresis widths in A scale with temperature and nanocube size in the same way as in the Ising representation, but the position in $\mathbf{B}$ of the critical point shifts with temperature and nanocube size (unlike for an inert wall in the Ising model, magenta line); it is therefore difficult to infer the experimental preference of either phase for the wall. C Furthermore, kinetic simulations reveal that in the lattice gas representation one transformation is rendered faster by decreasing system size, while the other is rendered slower. In experiment, and in the Ising model, both transformations are rendered faster by decreasing system size 


\section{a Ensemble average}
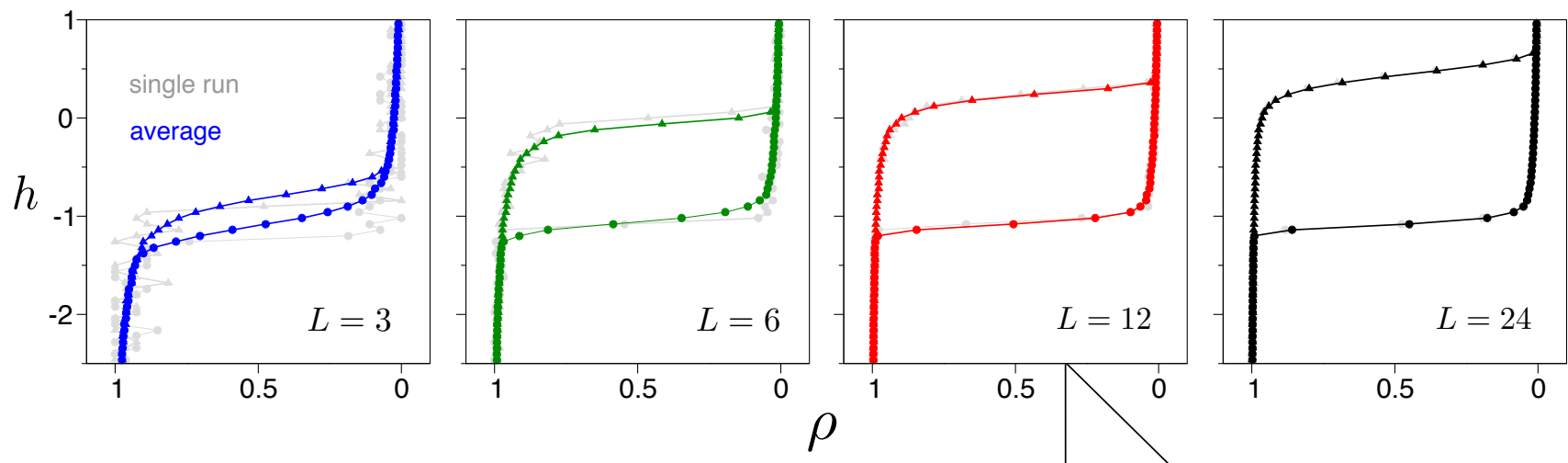

b Polydispersity
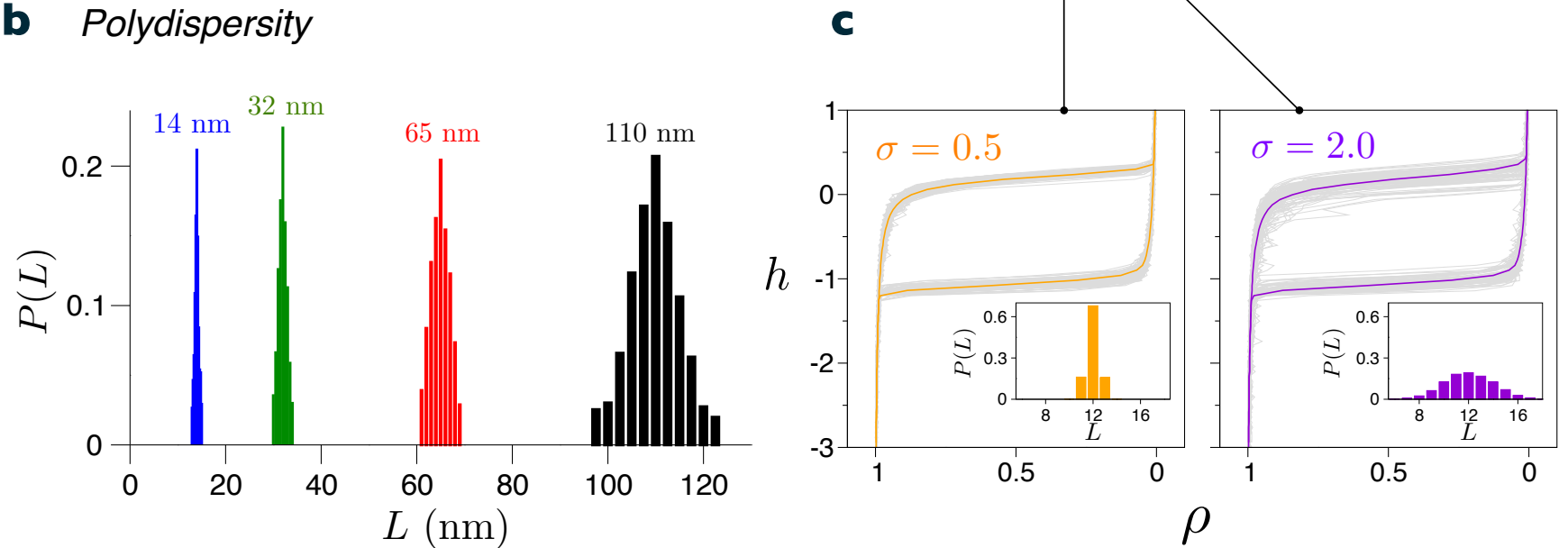

Figure S17. The statistics of nucleation ensure that ensemble averaged hysteresis loops are representative of phase transformations within individual cubes. A Magnetic field-magnetization hysteresis loops for the Ising model for a range of nanocube sizes. Shown are individual runs (grey) overlaid on the mean of 1000 runs (colors) (parameters: $\Delta=1$, temperature $=0.37 T_{c}$ ) As expected for nucleation, individual traces are stochastic, but trends inferred from the behaviors of the sample mean are similar to trends inferred from the behaviors of individual simulations. Also as expected, the smaller the cube, the broader the distribution of individual traces, and the more sloping are the mean plateaux. B Size distributions of nanocubes in experiment are narrow. The largest normalized standard deviation is $\sigma / \bar{L}=0.109$. C Simulations performed for a similar size distribution (a Gaussian of normalized standard deviation $\sigma / \bar{L}=0.04$ ) for a mean cube length $\bar{L}=12$ show that the mean is representative of the individual traces (here 1000 individual traces, in gray, are overlaid on their mean). The same conclusion holds even for a much broader distribution (a Gaussian of normalized standard deviation $\sigma / \bar{L}=0.17$ ). These results, and the knowledge that experimental hysteresis loops are very noisy when samples are not well-controlled (Fig. S18), give us confidence that ensemble averages measured in experiments (Fig. 2) identify behaviors representative of phase changes within individual nanocubes. 


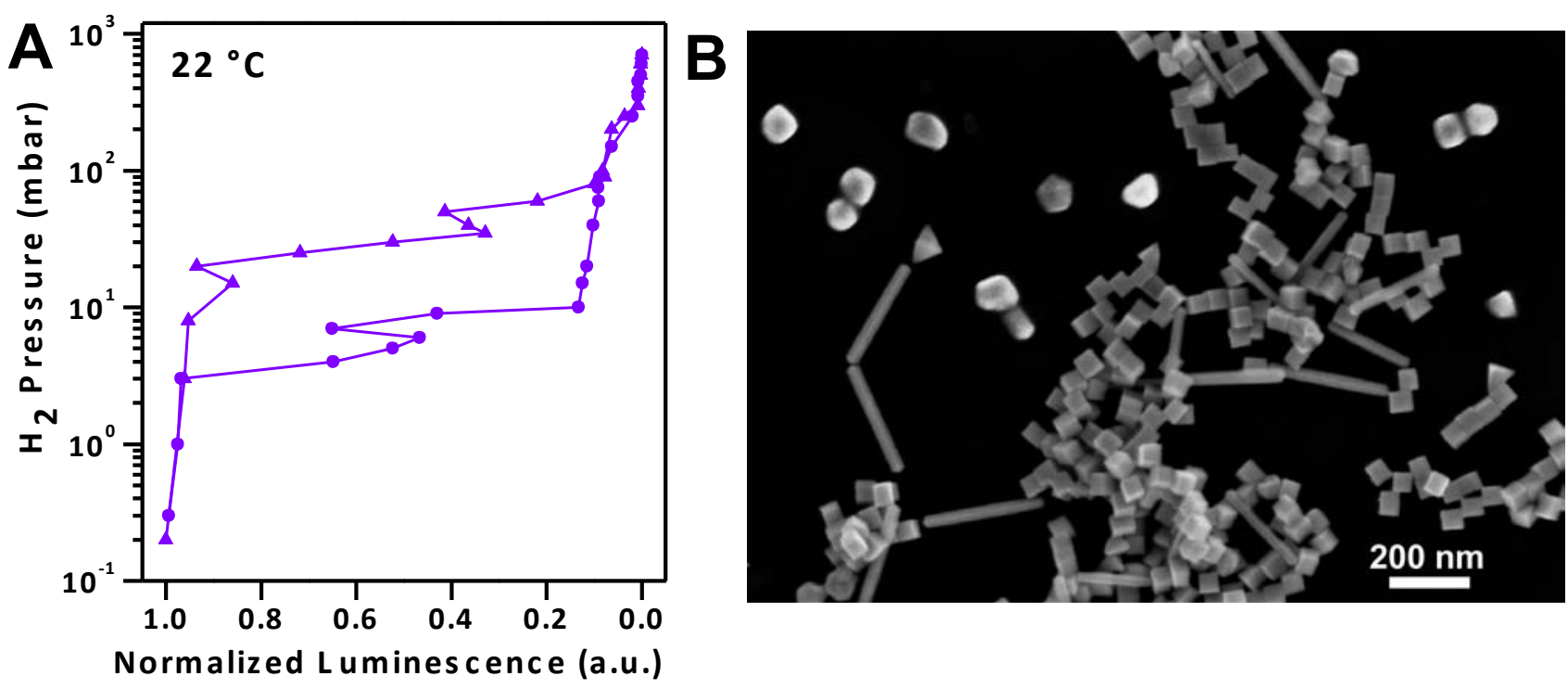

Figure S18. Pressure-Luminescence isotherm of polydisperse Pd sample with size and shape variation and corresponding SEM image of the polydisperse sample.

The P-L isotherm at $22{ }^{\circ} \mathrm{C}$ of a polydisperse Pd sample with a mixture of shapes and sizes of Pd is shown in Figure S18. In contrast to the single-valued luminescence values recorded for forward and reverse traces observed for the monodisperse nanocube samples (Figure 2, Figure S7), the isotherm of the polydisperse sample clearly demonstrates multi-valued luminescence at given equilibrium pressures during hydriding and dehydriding. This indicates that the luminescence values recorded during the phase transformation from metal to metal hydride occurs at several equilibrium pressures in the polydisperse samples; this is attributable to the multiple thermodynamically distinct sub-populations present due to large size and shape variation in the sample. 

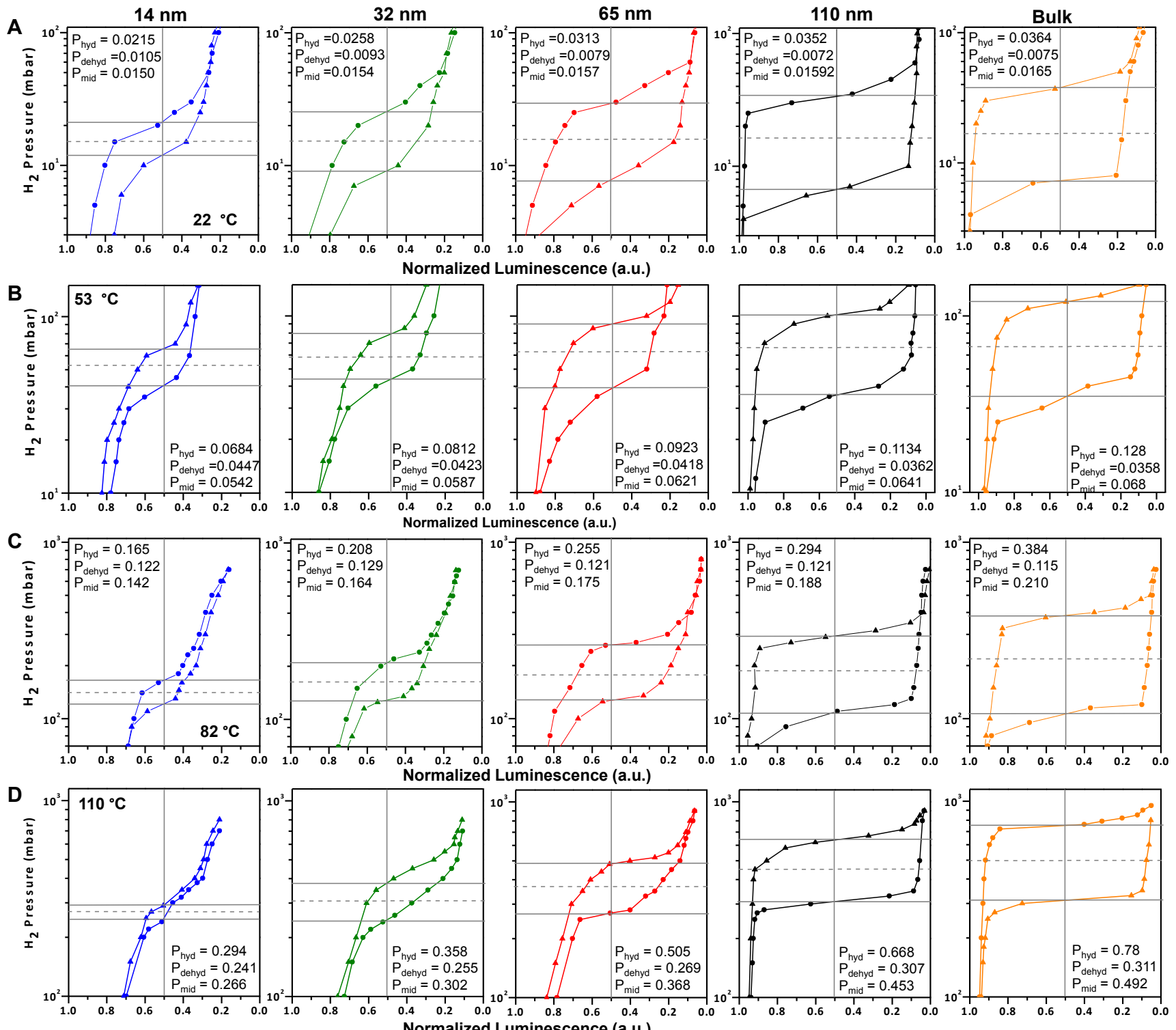

Figure S19. Magnified P-L isotherms of Pd nanocubes of all sizes and bulk at (A) $22{ }^{\circ} \mathrm{C}$, (B) $53{ }^{\circ} \mathrm{C},(\mathrm{C})$ $82{ }^{\circ} \mathrm{C}$, and (D) $110^{\circ} \mathrm{C}$. The isotherms are magnified to clearly represent the trend that as nanocube size increases the midpoint pressure $\left(\mathrm{P}_{\mathrm{mid}}\right)$ increases as well. This trend is more pronounced as the temperature increases from 22 to $110^{\circ} \mathrm{C}$. The hydriding pressure $\left(\mathrm{P}_{\text {hyd }}\right)$, dehydriding pressure $\left(\mathrm{P}_{\text {dehyd }}\right)$, and $\mathrm{P}_{\text {mid }}$ are indicated with horizontal gray lines evaluated at luminescence 0.5 shown by vertical gray line. $\operatorname{Ln}\left(\mathrm{P}_{\text {mid }}\right)$ was calculated by averaging $\operatorname{Ln}\left(\mathrm{P}_{\text {hyd }}\right)$ and $\operatorname{Ln}\left(\mathrm{P}_{\text {dehyd }}\right)$. Pmid was then calculated as $\mathrm{P}_{\text {mid }}=\mathrm{e}^{\operatorname{Ln}(\mathrm{Pmid})}$. 

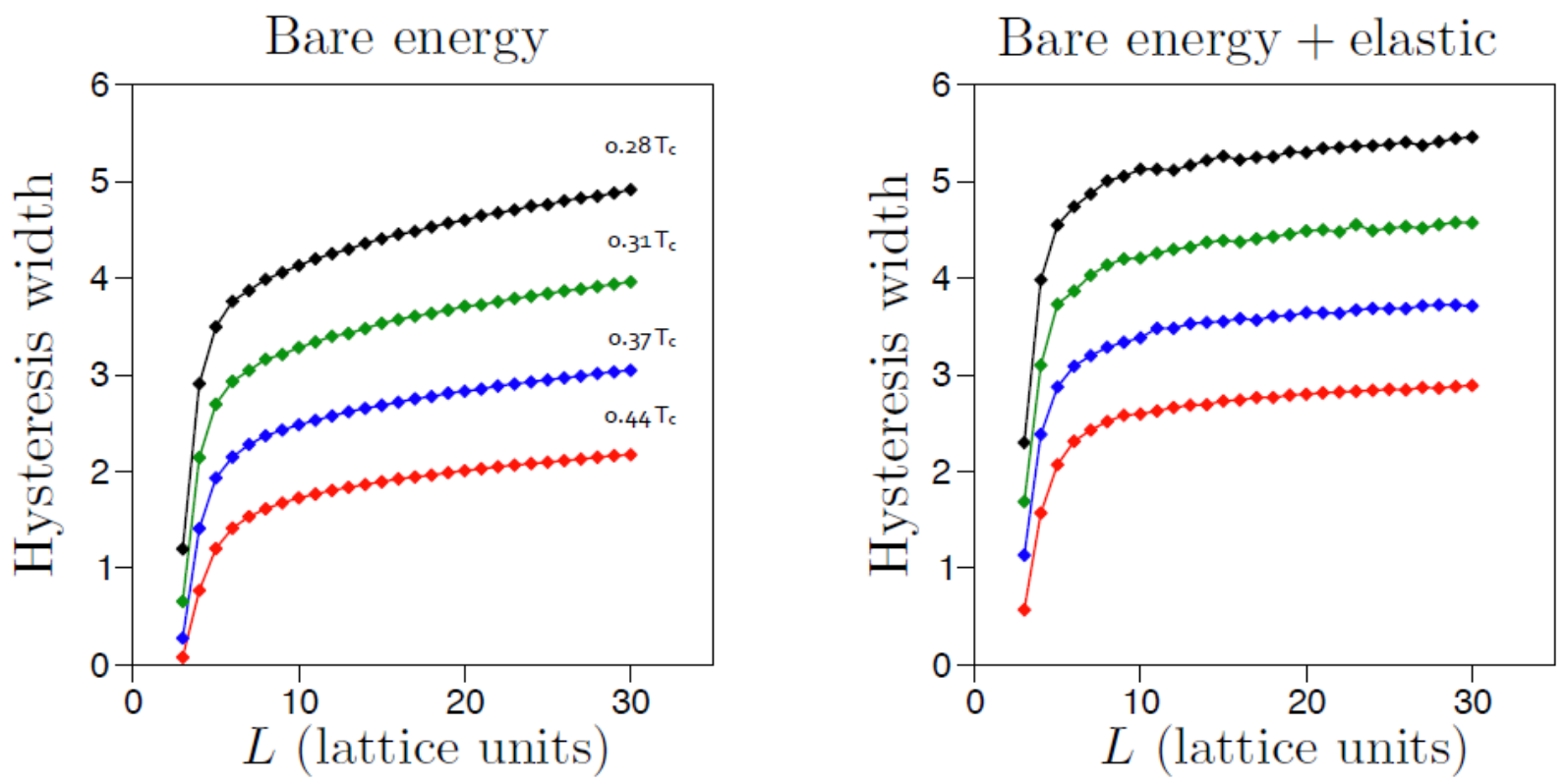

Figure S20. The scaling of hysteresis loop width with temperature and nanocube size seen in the Ising model (left) is qualitatively unchanged by the addition of a long-ranged elastic interaction (right): smaller nanocubes and higher temperatures result in reduced hysteresis.
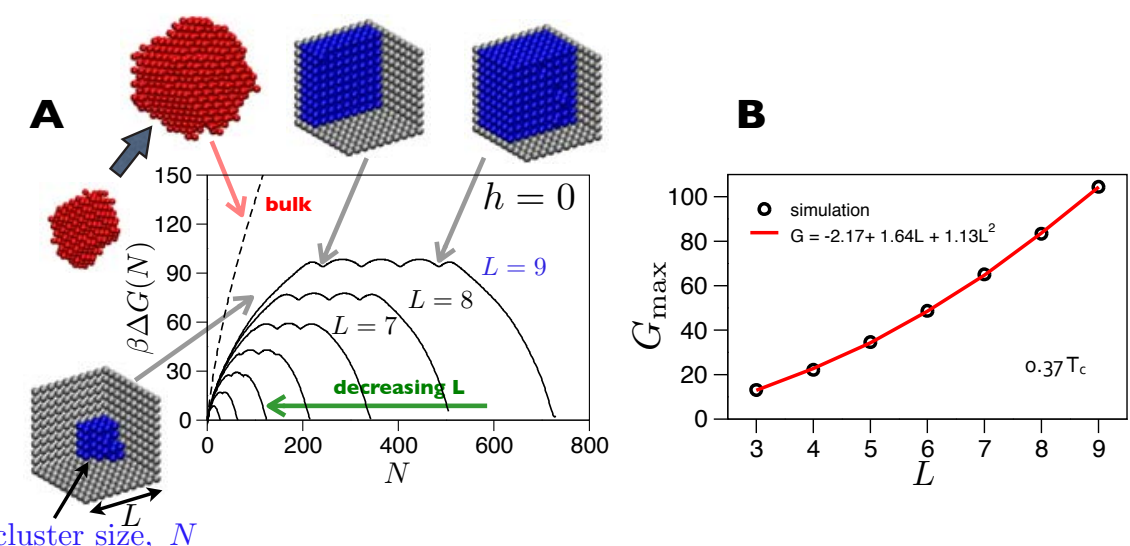

cluster size, $N$

C
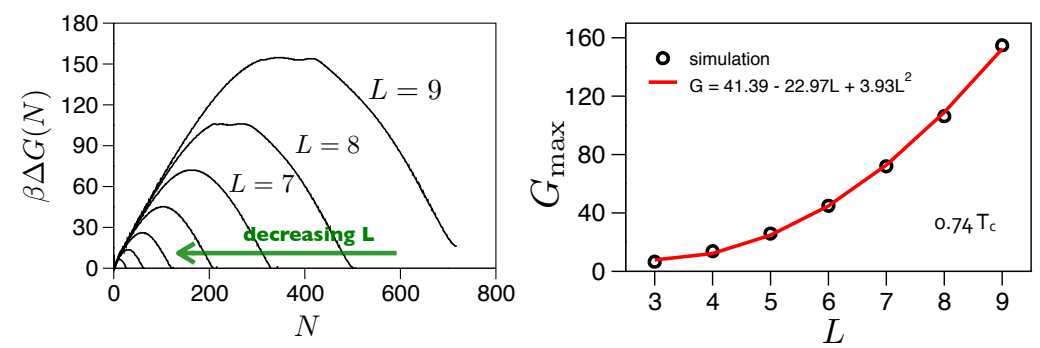

Figure S21. The scaling of nucleation free energy barriers with nanocube size seen in the Ising model at phase coexistence $(\mathrm{A}, \mathrm{B})$ is qualitatively unchanged by the addition of a long-ranged elastic interaction $(\mathrm{C}$, D). Barrier height is controlled by the surface tension between old and new phases, which is generated by the nearest-neighbor interaction. Although the mean-field term washes out some of the structure (the 
wiggles) associated with growing a nucleus layer-by-layer, it does not change the fact that barriers at coexistence are controlled by the size of the nanocube.
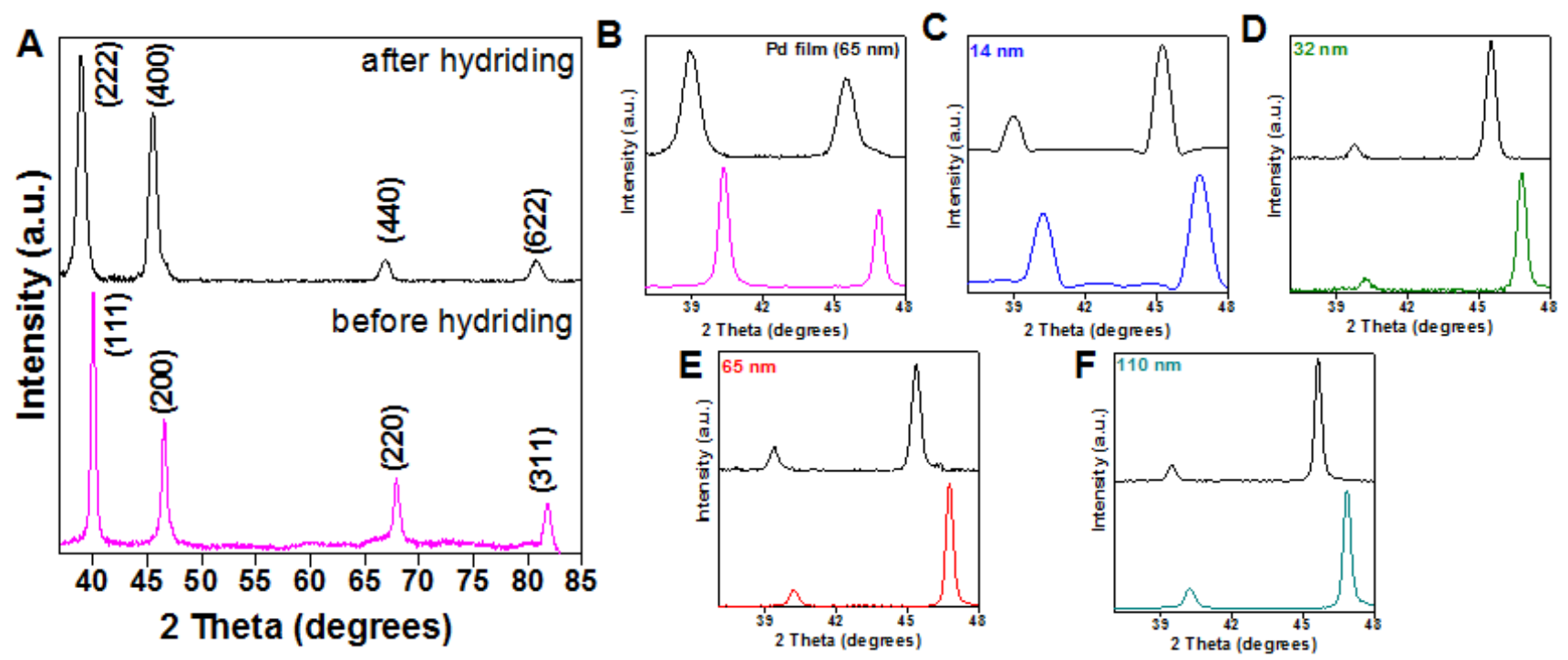

Figure S22. XRD patterns before and after hydriding of (A-B) $65 \mathrm{~nm}$ thick Pd film and (C-F) Pd nanocubes of various sizes. The size of the $\mathrm{Pd}$ nanocubes is provided in each graph. The bottom pattern in each graph is pristine $\mathrm{Pd}$ before hydriding and top graph is after hydriding. (B-F) Shows a higher magnification of the two strongest peaks used to calculate strain in the Pd nanocubes and film. Note: the low magnification XRD patterns of Pd nanocubes are provided in Figure S13. 
Table S3: The full width at half maximum (FWHM) of the XRD peaks shown in Fig. S22 B-F and the corresponding calculated strain of the nanocubes of different sizes and of Pd film before and after hydriding.

\begin{tabular}{|c|c|c|c|c|c|c|}
\hline $\begin{array}{l}\text { Size } \\
(\mathrm{nm})\end{array}$ & $\begin{array}{l}\text { FWHM before } \\
\text { hydriding at } 2 \theta \\
=38.9\end{array}$ & $\begin{array}{l}\text { Strain at } 2 \theta= \\
38.9 \text { before } \\
\text { hydriding }\end{array}$ & $\begin{array}{l}\text { FWHM after } \\
\text { hydriding at } 2 \theta= \\
38.9\end{array}$ & $\begin{array}{l}\text { Strain at } 2 \theta=38.9 \\
\text { after hydriding }\end{array}$ & $\begin{array}{l}\text { \% increase } \\
\text { in Strain }\end{array}$ & $\begin{array}{l}\text { E }_{\varepsilon} \text { after } \\
\text { hydriding } \\
\left(x 10^{-6}, \mathrm{eV} / \AA^{3}\right)\end{array}$ \\
\hline 14 & 0.84 & 0.00293748 & 0.93 & 0.003925955 & 33.65 & 4.665 \\
\hline 32 & 0.53 & 0.00329298 & 0.58 & 0.003910778 & 18.76 & 5.393 \\
\hline 65 & 0.43 & 0.00371025 & 0.55 & 0.005069407 & 36.63 & 9.624 \\
\hline 110 & 0.35 & 0.00337746 & 0.46 & 0.0044895 & 32.92 & 7.548 \\
\hline $\begin{array}{l}\text { Film } \\
(65 \mathrm{~nm})\end{array}$ & 0.46 & 0.00408093 & 0.84 & 0.008899737 & 118.08 & 29.66 \\
\hline $\begin{array}{l}\text { Size } \\
(\mathbf{n m})\end{array}$ & $\begin{array}{l}\text { FWHM before } \\
\text { hydriding at } 2 \theta \\
=45.4\end{array}$ & $\begin{array}{l}\text { Strain at } 2 \theta= \\
45.4 \text { before } \\
\text { hydriding }\end{array}$ & $\begin{array}{l}\text { FWHM after } \\
\text { hydriding at } 2 \theta= \\
45.4\end{array}$ & $\begin{array}{l}\text { Strain at } 2 \theta=45.4 \\
\text { after hydriding }\end{array}$ & $\begin{array}{l}\text { \% increase } \\
\text { in Strain }\end{array}$ & $\begin{array}{l}\mathbf{E}_{\varepsilon} \text { after } \\
\text { hydriding } \\
\left(\mathrm{x} 10^{-6}, \mathrm{eV} / \AA^{3}\right)\end{array}$ \\
\hline 14 & 0.8 & 0.001920891 & 0.88 & 0.0032661 & 70.03 & 3.229 \\
\hline 32 & 0.48 & 0.002195073 & 0.56 & 0.003591202 & 63.60 & 4.547 \\
\hline 65 & 0.36 & 0.002369605 & 0.45 & 0.003921705 & 65.50 & 5.759 \\
\hline 110 & 0.28 & 0.002101246 & 0.36 & 0.003479949 & 65.61 & 4.535 \\
\hline $\begin{array}{l}\text { Film } \\
(65 \mathrm{~nm})\end{array}$ & 0.39 & 0.002682224 & 0.83 & 0.008616947 & 221.26 & 27.806 \\
\hline
\end{tabular}

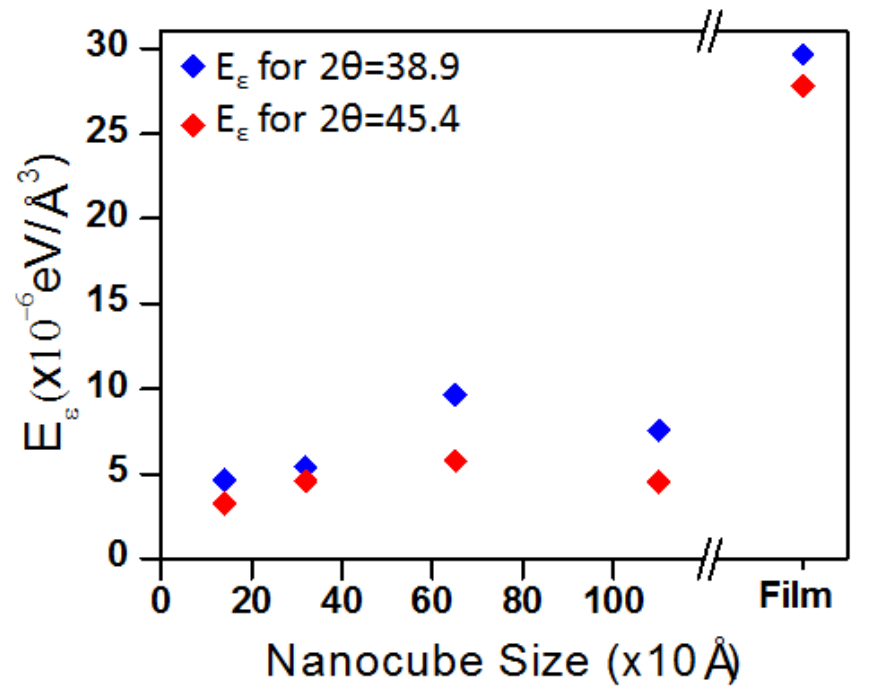

Figure S23. The elastic strain energy calculated from XRD data at $2 \theta=38.9$ and 45.4 is shown as a function nanocube size. The strain energy represented here is after hydriding the Pd nanocubes and film. 
The strain in the Pd film and Pd nanocubes was calculated by examining the FWHM of the XRD peaks at (111) and (200) before hydriding and at (222) and (400) after hydriding using a modified Scherrer's equation given $b^{24}$,

$$
\beta_{t}=\left(\frac{0.9 \lambda}{D \cos \theta}\right)+(4 \varepsilon \tan \theta) .
$$

Here $\beta_{t}$ is total broadening of the XRD peak in radians, $\lambda$ is wavelength of $\mathrm{Cu} \mathrm{K} \alpha$ line $\lambda=1.5418 \AA, D$ is the size of the nanocrystal in $\AA, \theta$ is half of the $2 \theta$ values where the peaks are observed in radians, and $\varepsilon$ is strain. Strain was calculated at $\theta=19.45$, and $\theta=22.72$ before and after hydriding and the $\%$ increase in strain was evaluated by examining the difference in strain before and after hydriding. The \% increase in strain clearly exemplifies the strain induced in the film after $\mathrm{Pd}$ converts to $\mathrm{PdH}_{\mathrm{x}}$ is substantially larger than those induced in nanocubes. This is attributable to clamping effects of the Pd film to the substrate. The $\%$ increase in strain clearly demonstrates that the strain induced in the nanocubes do not show a noticeable size-dependent trend indicating that the observed transformations are a direct consequence of thermally-driven phase transitions and not induced by strain.

The elastic strain energy $\left(\mathrm{E}_{\varepsilon}\right)$ after hydriding was calculated by, $E_{\varepsilon}=\frac{1}{2} Y \varepsilon^{2}$ where $\mathrm{Y}$ is the sizedependent Young's modulus. ${ }^{25}$ The values for Young's modulus were obtained from ref. 25 . The trend observed in Figure S23 demonstrates that the $65 \mathrm{~nm}$ Pd film has $3-6$ fold more strain energy relative to the nanocubes. This is attributable to the clamping of thin films to the supporting substrate which induces excessive strain due to volumetric expansion and contraction during hydriding and dehydriding. The induced strain often results in peeling of the film from the substrate surface, which was also observed in our experiments. Nanocubes retained their shape and integrity after multiple cycles of hydriding (Fig.S2). 
phase diagram

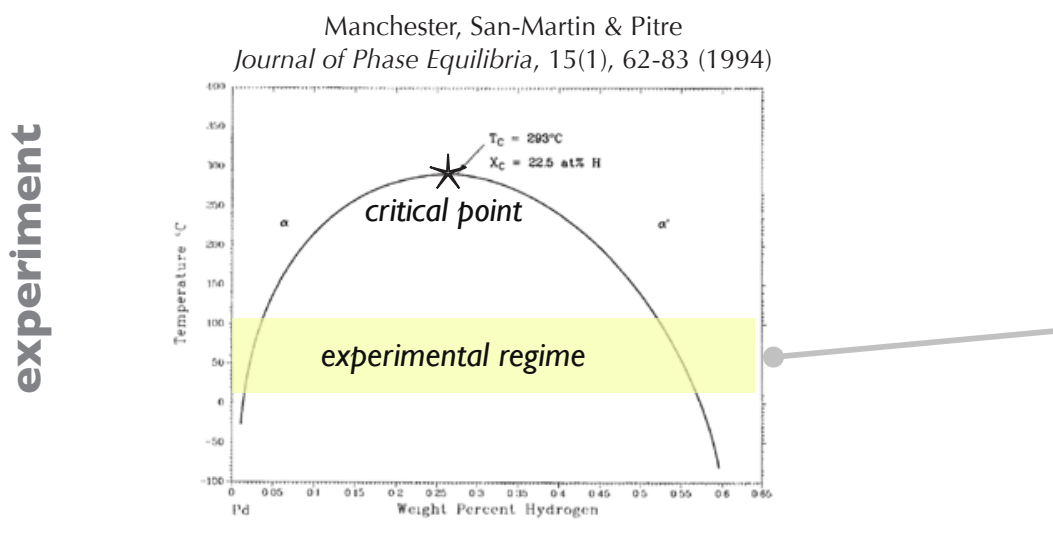

Fig. 1 Assessed Pd-H phase diagram. $T-X$ projection from a $P$-X-T surface onto a plane at $P=10^{2} \mathrm{~Pa}$. hysteresis scaling

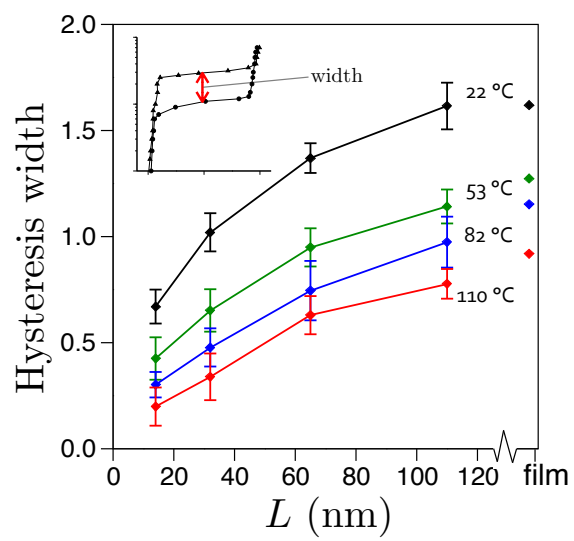

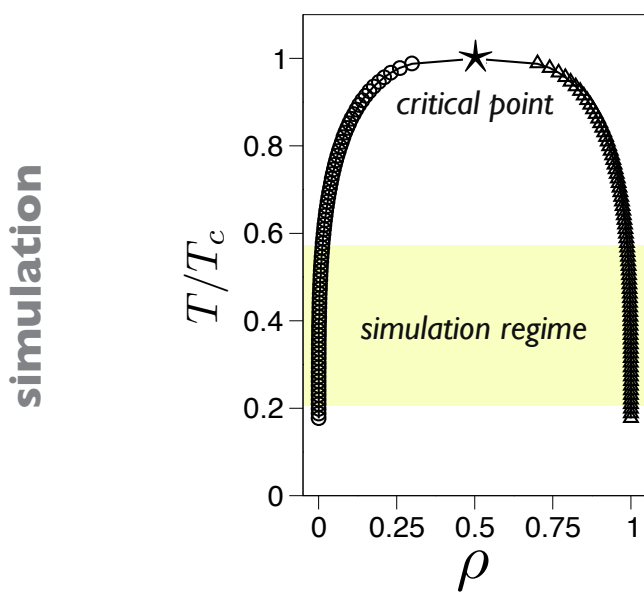

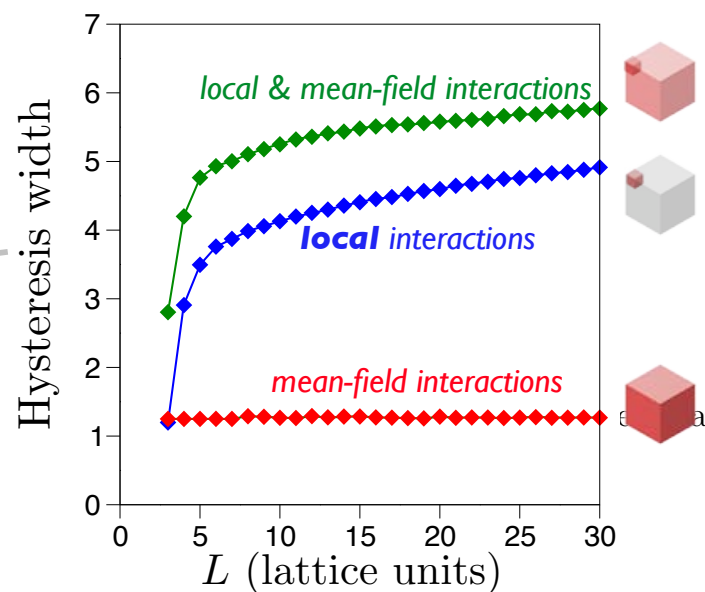

Figure S24. Local interactions capture the physics appropriate to our experimental regime. Top left: Pd $\mathrm{H}$ phase diagram taken from the classic literature and marked to show the regime in which our experiments take place. ${ }^{26}$ This regime is well below the critical temperature. Top right: The scaling of hysteresis with nanocube size in our experiments shows that phase change happens more readily in small nanocubes. Bottom left: Ising model simulation phase diagram marked as its experimental counterpart. Bottom right: scaling of hysteresis with nanocube size in simulations reproduces the experimental trend only if local interactions are considered. Local interactions give rise to a surface tension between phases. Nonlocal mean-field interactions known to be crucial near the critical point are only a perturbation in the regime appropriate to our experiments. A similar conclusion was drawn in Refs. 27 and 28 of the main text.

\section{References}

1 Zoltowski, P. \& Makowska, E. Diffusion coefficient of hydrogen in $\alpha$-phase palladium and palladium platinum alloy. Phys. Chem. Chem. Phys. 3, 2935-2942 (2001).

2 Fedorovich, R. D., Naumovets, A. G. \& Tomchuk, P. M. Electron and light emission from island metal films and generation of hot electrons in nanoparticles. Physics Reports 328, 73-179 (2000).

3 Varnavski, O. P., Mohamed, M. B., El-Sayed, M. A. \& GoodsonIII, T. Relative enhancement of ultrafast emission in gold nanorods. J. Phys. Chem. B 107, 3101-3104 (2003). 
Beversluis, M. R., Bouhelier, A. \& Novotny, L. Continuum generation from single gold nanostructures through near-field mediated intraband transitions. Phys. Rev. B 68, 115433 (2003). Magde, D., Wong, R. \& Seybold, P. G. Fluorescence quantum yields and their relation to lifetimes of rhodamine $6 \mathrm{G}$ and fluorescein in nine solvents: Improved absolute standards for quantum yields. Photochem. and Photobiol. 75, 327-334 (2002).

Eastman, D. E., Cashion, J. K. \& Switendick, A. C. Photoemission studies of energy levels in the palladium-hydrogen system. Phys. Rev. Lett. 27, 35-38 (1971).

Wicke, E. Electronic structure and properties of hydrides of $3 \mathrm{~d}$ and $4 \mathrm{~d}$ metals and intermetallics. $J$. Less-Comm. Metals 101, 17 - 33 (1984).

Xu, X., Chen, P. \& Goodman, D. W. A comparative study of the coadsorption of CO and NO on $\operatorname{Pd}(100), \operatorname{Pd}(111)$, and silica-supported palladium particles with infrared reflection-absorption spectroscopy J. Phys. Chem. 98, 9242-9246 (1994).

Luther, J. M., Jain, P. K., Ewers, T. \& Alivisatos, A. P. Localized surface plasmon resonances arising from free carriers in doped quantum dots. Nature Mater. 10, 361-366 (2011).

Langhammer, C., Zhdanov, V. P., Zoric', I. \& Kasemo, B. Size-dependent kinetics of hydriding and dehydriding of Pd nanoparticles. Phys. Rev. Lett. 104, 135502 (2010).

1 Langhammer, C., Larsson, E. M., Kasemo, B. \& Zoric', I. Indirect nanoplasmonic sensing: ultrasensitive experimental platform for nanomaterials science and optical nanocalorimetry. Nano Lett. 10, 3529-3538 (2010).

Piao, Y., Jang, Y., Shokouhimehr, M., Lee, I. S. \& Hyeon, T. Facile aqueous-phase synthesis of uniform palladium nanoparticles of various shapes and sizes. Small 3, 255-260 (2007).

3 Efremenko, I. Implication of palladium geometric and electronic structures to hydrogen activation on bulk surfaces and clusters. J. Mol. Catalysis A 173, 19-59 (2001).

4 Okuyama, H., Siga, W., Takagi, N., Nishijima, M. \& Aruga, T. Path and mechanism of hydrogen absorption at Pd(100). Surf. Sci. 401, 344-354 (1998).

5 Narayanan, R. \& El-Sayed, M. A. Shape-dependent catalytic activity of platinum nanoparticles in colloidal solution. Nano Lett. 4, 1343-1348 (2004).

Zoric', I., Larsson, E. M., Kasemo, B. \& Langhammer, C. Localized surface plasmons shed light on nanoscale metal hydrides. Adv. Mater. 22, 4628-4633 (2010).

1 Rikvold, P. A., Tomita, H., Miyashita, S. \& Sides, S. W. Metastable lifetimes in a kinetic Ising model: Dependence on field and system size. Phys. Rev. E 49, 5080 (1994).

8 Palsson, G. K. et al. Hydrogen site occupancy and strength of forces in nano-sized metal hydrides. Phys. Rev. B. 85, 195407 (2012).

9 Pundt, A. \& Kirchheim, R. Hydrogen in metals: microstructural aspects. Annu. Rev. Mater. Res. 36, 555-608 (2006).

Feenstra, R., Groot, D. G. d., Rector, J. H., Salomons, E. \& Griessen, R. Gravimetrical determination of pressure-composition isotherms of thin $\mathrm{PdH}_{\mathrm{C}}$ films. J. Phys. F: Met. Phys. 16, 1953-1963 (1986).

1 Peden, C. H. F., Kay, B. D. \& Goodman, D. W. Kinetics of hydrogen absorption by chemically modified Pd(110). Surface Science 175, 215-225 (1986).

2 Blackford, L., Arnold, C. S., Mulhern, P. J. \& Jericho, M. H. A scanning tunneling microscope study of a palladium sphere in hydrogen gas: expansion and surface topology. J. Appl. Phys. 76, 4054-4060 (1994).

3 Sun, Y. \& Xia, Y. Shape-controlled synthesis of gold and silver nanoparticles. Science 298, 21762179 (2002). 
24 Mote, V. D., Purushotham, Y. \& Dole, B. N. Williamson-Hall analysis in estimation of lattice strain in nanometer-sized ZnO particles. J. Theor. and Appl. Phys. 6, 1-8 (2012).

25 Sharma, P. \& Ganti, S. On the grain-size-dependent elastic modulus of nanocrystalline materials with and without grain-boundary sliding. J. Mater. Res. 18, 1823-1826 (2003).

26 Manchester, F. D., San-Martin, A. \& Pitre, J. M. The H-Pd (hydrogen-palladium) system. J. Phase Equilibria 15, 62-83 (1994). 